\title{
USER UNDERSTANDING OF INTERNET DATA BREACHES
}

by

Zahra Hassanzadeh

Submitted in partial fulfillment of the requirements for the degree of Master of Science, Computer Science

at

\author{
Carleton University \\ Ottawa, Canada
}

August 2019

(c) Copyright by Zahra Hassanzadeh, 2019 


\section{Table of Contents}

List of Tables . . . . . . . . . . . . . . . . . . . . . . . v v

List of Figures $\ldots \ldots \ldots \ldots \ldots \ldots \ldots$ vi

Abstract ................................. ix

Acknowledgements ....................... . . .

Chapter 1 Introduction . . . . . . . . . . . . . . . 1

1.1 Motivation . . . . . . . . . . . . . . . . . . . . . 1

1.2 Research Goal . . . . . . . . . . . . . . . . . . . . . . . 3

1.2 .1 Research Questions . . . . . . . . . . . . . . . . . 3

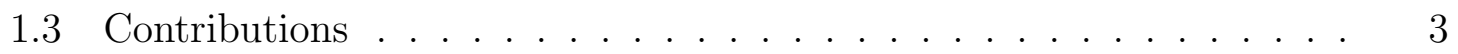

1.4 Thesis Outline. . . . . . . . . . . . . . . . . . . . 4

Chapter 2 Background . . . . . . . . . . . . . . . . 6

2.1 Overview of Computer Attacks and Intrusions $\ldots \ldots \ldots \ldots$

2.2 Intrusion Detection Systems (IDSs) $\ldots \ldots \ldots \ldots \ldots \ldots$

2.2 .1 Taxonomy of Intrusion Detection Systems $\ldots \ldots \ldots$

2.2 .2 Classification of Detection $\ldots \ldots \ldots \ldots \ldots$

$2.3 \quad$ Anatomy of a Cyber Security Breach . . . . . . . . . . . . . . 11

2.3 .1 What cause an Internet data breach? . . . . . . . . . . . 12

2.3 .2 What happens in an Internet data breach? . . . . . . . . . . 13

2.3 .3 CERT \& CVE . . . . . . . . . . . . . . . . . . . . . . . . . 13

2.3 .4 What should companies do? . . . . . . . . . . . . . . . 14

2.3 .5 What should the customers do? . . . . . . . . . . . 15

$2.4 \quad$ Data Breaches and Crisis Response $\ldots \ldots \ldots \ldots \ldots$

2.4 .1 Narrative-semiotic approach $\ldots \ldots \ldots \ldots$

2.5 Users' Mental Model of Privacy and Security . . . . . . . . . . . 20

Chapter $3 \quad$ Mental Models of Internet Data Breaches . . . . . . . . 24

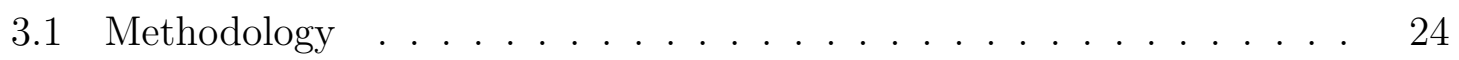

3.1 .1 Session $\ldots \ldots \ldots \ldots \ldots$

3.1 .2 Participants . . . . . . . . . . . . . . . . . 27 
3.2 Data Analysis $\ldots \ldots \ldots \ldots$

3.3 Results . . . . . . . . . . . . . . . . . . . . . . . . . . . . 30

$3.3 .1 \quad$ SeBIS Questions Results . . . . . . . . . . . . . . . 30

3.3 .2 Questionnaire Results . . . . . . . . . . . . . . . . 30

3.3 .3 Drawing Task . . . . . . . . . . . . . . . . . . . . 38

3.4 Discussion . . . . . . . . . . . . . . . . . . . . . . . . . . . 43

3.4 .1 Drawing and open questions analysis $\ldots \ldots \ldots$. . . . . 43

3.4 .2 Questionnaire analysis $\ldots \ldots \ldots \ldots \ldots$

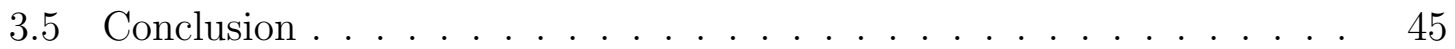

Chapter 4 Study 2: Crisis Communication Analysis $\ldots \ldots$. . . 47

4.1 Methodology . . . . . . . . . . . . . . . . . . . . . . . . . . . . 47

$4.1 .1 \quad$ Coding . . . . . . . . . . . . . . . . . . . . . . . . . . . . . 49

4.1 .2 Overview . . . . . . . . . . . . . . . . . . 50

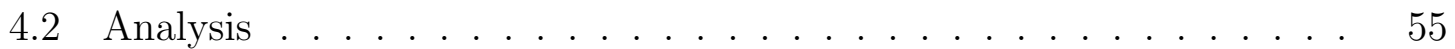

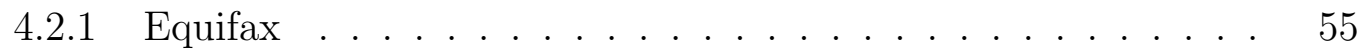

4.2 .2 Marriott Hotels . . . . . . . . . . . . . . . . . . . . . . . . 67

4.2 .3 Discussion $\ldots \ldots \ldots \ldots \ldots \ldots$

4.2 .4 Conclusion $\ldots \ldots \ldots \ldots$

Chapter 5 Study 3: Quantitative Study on Internet Data Breaches 79

5.1 Introduction $\ldots \ldots \ldots \ldots \ldots \ldots \ldots \ldots$

5.1 .1 Research questions . . . . . . . . . . . . . . . . . 79

5.2 Methodology $\ldots \ldots \ldots \ldots \ldots \ldots$

5.2 .1 Questionnaire . . . . . . . . . . . . . . . . . . 80

5.2 .2 Participants . . . . . . . . . . . . . . . . . . 83

5.3 Data Analysis $\ldots \ldots \ldots \ldots \ldots$

5.4 Results . . . . . . . . . . . . . . . . . . . . . . . . . . . . 84

$5.4 .1 \quad$ SeBIS Questions Results . . . . . . . . . . . . . . . . . 84

5.4 .2 Security Awareness . . . . . . . . . . . . . . . . . . . . . . 84

5.4 .3 Data Breach Questions . . . . . . . . . . . . . . . . . . 86

5.4 .4 Data Breach Description . . . . . . . . . . . . . . . . . 90

5.5 Discussion $\ldots \ldots \ldots \ldots \ldots$

5.6 Conclusion $\ldots \ldots \ldots \ldots \ldots \ldots$ 
Chapter 6 Conclusions $\ldots \ldots \ldots$. . . . . . . . . . . . . . . . . 98

6.1 Main Findings $\ldots \ldots \ldots \ldots$

6.1.1 Study 1: Exploring Mental Models of Internet Data Breaches. 98

6.1 .2 Study 2: Crisis Communication Analysis $\ldots . . . \ldots . . . .99$

6.1.3 Study 3: Quantitative study on Internet Data Breaches . . . . 100

6.1 .4 Combining Insight and implications . . . . . . . . . . . . 100

6.2 Limitations . . . . . . . . . . . . . . . . . . . . . . . . . 101

6.3 Contributions . . . . . . . . . . . . . . . . . . . . . . 102

6.4 Future Work . . . . . . . . . . . . . . . . . . . . . . . . 103

Bibliography . . . . . . . . . . . . . . . . . . . . . 104

Appendix A Study 1: Recruitment Poster, Consent Form, Questionnaire $\ldots \ldots \ldots \ldots \ldots \ldots \ldots \ldots \ldots \ldots$

Appendix B Study 2: Equifax (Nodes, Extract of summary by Code

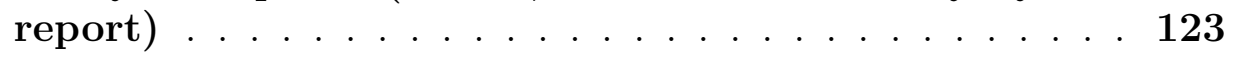

Appendix C Study 2: Marriott Hotels (Extract of summary by Code

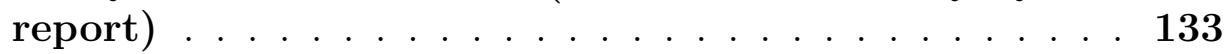

Appendix D Study 3: Consent Form, Questionnaire . . . . . . . . . . 140 


\section{List of Tables}

$3.1 \quad$ SeBIS questions. . . . . . . . . . . . . . . 26

$3.2 \quad$ Open-ended questions from the post-test questionnaire . . . . . 27

$3.3 \quad$ Study participants $($ Total $=20) \ldots \ldots \ldots$. . . . . . . 28

\begin{tabular}{ll}
\hline .4 & How would you know if you have been affected in an Internet \\
\hline & data breach? $\ldots \ldots \ldots \ldots \ldots . \ldots \ldots$
\end{tabular}

$3.5 \quad$ Participants extracted comments for causes of a data breach . . 35

$4.1 \quad$ Equifax: number of stories per publication and timeline . . . . 48

$4.2 \quad$ Marriott Hotels: number of stories per publication and timeline 48

$4.3 \quad$ Image repair strategies in Equifax press release . . . . . . . . . 55

$4.4 \quad$ Image repair strategies in the Marriott Hotels' press release . . 67

$5.1 \quad$ Security awareness questions . . . . . . . . . . . . . . . 81

$5.2 \quad$ Data breach description . . . . . . . . . . . . . . 82

$5.3 \quad$ Participants' demographics . . . . . . . . . . . . . . . 84

$5.4 \quad$ Wilcoxon test on data breach descriptions (company vs news) . 92

$5.5 \quad$ ANOVA test on the data breach description . . . . . . . . . . . 94 


\section{List of Figures}

$1.1 \quad$ Types of insider threats and their risk level . . . . . . . . 2

$2.1 \quad$ Taxonomy of intrusion detection systems . . . . . . . . . . . . 10

$3.1 \quad$ SeBIS questions . . . . . . . . . . . . . . . . . . . 31

$3.2 \quad$ SeBIS questions $\ldots \ldots \ldots \ldots$

$3.3 \quad$ World's Biggest Data Breaches \& Hacks . . . . . . . . . . . . 33

$3.4 \quad$ What causes an Internet data breach? . . . . . . . . . . . . 36

$3.5 \quad$ What are the consequences of Internet data breach? . . . . . 37

$3.6 \quad$ Why do data breaches happen? . . . . . . . . . . . . . . 38

$3.7 \quad$ How likely is it to prevent a data breach via the following practices? . . . . . . . . . . . . . . . 39

3.8 P19. Attacker hack the admin system to steal identity verification data. . . . . . . . . . . . . . . . 40

$3.9 \quad$ P14. Attacker hack the user system to steal personal data. . . 41

$3.10 \quad$ P5. Backdoor access. . . . . . . . . . . . . . . . . . 42

$4.1 \quad$ Image repair nodes sample . . . . . . . . . . . . . . . 50

$4.2 \quad$ Narrative-semiotics nodes sample . . . . . . . . . . . . . 51

$4.3 \quad$ Actantial model of Equifax data breach extracted from press releases (narrative program 1) $\ldots \ldots \ldots . . \ldots 59$

$4.4 \quad$ Actantial model of Equifax data breach extracted from press releases (narrative program 2) $\ldots \ldots \ldots$. . . . . . . . . 61

$4.5 \quad$ Actantial model of Equifax data breach extracted from press releases (narrative program 3 ) $\ldots \ldots \ldots 6$. . . . . . . 62

$4.6 \quad$ Actantial model of Equifax data breach . . . . . . . . . . . 63

$4.7 \quad$ Actantial model of Equifax data breach extracted from news and GAO report . . . . . . . . . . . . . 66 
$4.8 \quad$ Actantial model of Equifax data breach extracted from news and GAO report (narrative program 2) $\ldots$. . . . . . . . 66

$4.9 \quad$ Actantial model of Marriott Hotels data breach extracted from company's press release . . . . . . . . . . . . 71

$4.10 \quad$ Actantial model of Marriott Hotels data breach extracted from news and class action complaint . . . . . . . . . . 73

$4.11 \quad$ Actantial model of Marriott Hotels data breach extracted from news and class action complaint . . . . . . . . . . . 74

$4.12 \quad$ Equifax: comparing narrative program 2 . . . . . . . . 75

$4.13 \quad$ Equifax: comparing narrative program 1 . . . . . . . . . . 76

$4.14 \quad$ Marriott: comparing narrative program . . . . . . . . . . . . . 77

$5.1 \quad$ SeBIS questions . . . . . . . . . . . . . . . . . 85

$5.2 \quad$ Security awareness . . . . . . . . . . . . . . 86

$5.3 \quad$ How would you rate the accountability of the following people for data protection in an enterprise data breach? . . . . . . . . 87

$5.4 \quad$ Which of the following are causes of data breach? . . . . . . . 88

$5.5 \quad$ What are the consequences of a data breach? . . . . . . . . . 89

$5.6 \quad$ Why do data breaches happen? . . . . . . . . . . . . . . 90

$5.7 \quad$ How likely would the following practices prevent a data breach? 91

$5.8 \quad$ Data breach description . . . . . . . . . . . . . . . 93

$5.9 \quad$ SeBIS aggregated score, Mean=54.2, $\mathrm{Sd}=9.7$. . . . . . . . 96

$5.10 \quad$ SeBIS aggregated score, Mean $=59.47, \mathrm{Sd}=10.3$. . . . . . . 96 


\section{List of Abbreviations}

$\begin{array}{ll}\text { ANOVA } & \text { Analysis of variance } \\ C E O & \text { Chief Executive Officer } \\ C E R T & \text { Computer Emergency Response Team } \\ C E R T / C C & \text { Computer Emergency Response Team Coordination Center } \\ C S I R T & \text { Computer Security Incident Response Team } \\ C V E & \text { Common Vulnerability and Exposures } \\ D o S / D D o S & \text { Denial of Service/ Distributed Denial of Service } \\ F T C & \text { Federal Trade Commission } \\ G A O & \text { Government Accountability Office } \\ G D P R & \text { General Data Protection Regulation } \\ I D S & \text { Intrusion Detection Systems } \\ I S P & \text { Internet service provider } \\ I T & \text { Information Technology } \\ M A C & \text { Media Access Control address } \\ P I I & \text { Personally Identifiable Information } \\ S E B I S & \text { Security Behavior Intentions Scale } \\ U R L & \text { Uniform Resource Locator } \\ U S B & \text { Universal Serial Bus } \\ V P N & \text { Virtual Private Networks } \\ X S S & \text { Cross-Site Scripting attacks }\end{array}$




\begin{abstract}
Data breaches happen when information is accessed by an unauthorized party. They are growing rapidly and becoming a high-impact problem that raises concerns for individuals as well as companies. We explored users' understanding of Internet data breaches. First, we did a study with 20 participants to explore users' mental models of Internet data breaches. We found that participants have a basic model of how an Internet data breach happens but they have some uncertainties regarding system vulnerabilities, causes, consequences, prevention methods, and follow-up steps after a breach. We then analyzed different documents for data breach notifications and news. We found that companies use crisis communication strategies to reduce their reputational damage as well as financial loss. We next conducted a quantitative study of 100 participants in an online survey to check the generality of our results from the first study and our document analysis. We found that the extracted mental models from our first study can not be generalized to a bigger public. Our findings of this study regarding the influence of the communication strategies was in line with our second study, and suggest that it affects the users' perception of victimization, attitudes in data protection, and responsibility. All in all, people's mental models of Internet data breaches differ and they could depend on their level of expertise in security due to their education or their experience. Moreover, the way companies and news frame an incident affect people's mental model of data breaches.
\end{abstract}




\section{Acknowledgements}

I would like to express my gratitude to my awesome supervisor, Dr. Robert Biddle.

Without his encouragement, support and guidance this thesis would not have been completed. I am really lucky to have been one of his students.

I would like to thank you, Dr. Sky Marsen for your guidance and feedback during my crisis communication analysis. Thank you, Dr. Sonia Chiasson, and Dr. Hussein Al Osman for agreeing to be on my thesis committee. Thank you, Dr. Brian Greenspan for agreeing to chair my defense.

To my parents, Mahrokh and Teymour, and my siblings, Somayeh, Samira, Ali: thank you for your love, unfailing support, and continuous encouragement throughout my years of study.

To my sister, Dr. Samira: this would not have been completed if not for your support and kindness.

I am grateful to all my friends in Computer Science and HCI for our good times. 


\section{Chapter 1}

\section{Introduction}

\subsection{Motivation}

Nowadays, with the rapid development of information technology and massive dis-

tribution of digital information throughout the Internet, cybersecurity threats are growing rapidly and have aroused broad concern. A data breach is a successful malicious attack which leads to the compromise or the loss of data [81]. People's Personally Identifiable Information (PII) is often stored in the companies' database servers, where it is subject to the risk of misuse. According to the Breach Level Index report, 944 breach incidents happened during the first six months of 2018, where more than 18 million records were compromised or exposed every day. Only $2.2 \%$ of all the data leaked, lost, or stolen was encrypted [60]. These numbers show an increase of 72 percent over the first half of 2017. According to the European Union Agency for Cybersecurity, considering the geographic landscape, North America is regarded as the most popular target, representing $57 \%$ of the breaches [27].

Depending on the size, scale, and type of stolen information, the potential consequences of a data breach can be huge. A data breach can put people at risk of identity theft, which often happens through fraudulent use of existing accounts like credit cards, online accounts, and insurance. It can also lead to financial loss and emotional distress [91].

A data breach can be caused by an internal or external action, which can happen intentionally or inadvertently. Statistics from the Breach Level Index report showed that more than 40 percent of breach incidents occurred due to the accidental loss or a malicious insider in the first half of 2018 [60]. A recent survey illustrated that the groups shown in Figure 1.1 are among the high-risk for insider threat within a company or an organization. According to this survey, twenty-seven percent of organizations said insider attacks have become more frequent [14], which can be due to inappropriate policies and failure to apply preventive technologies [53]. This survey 


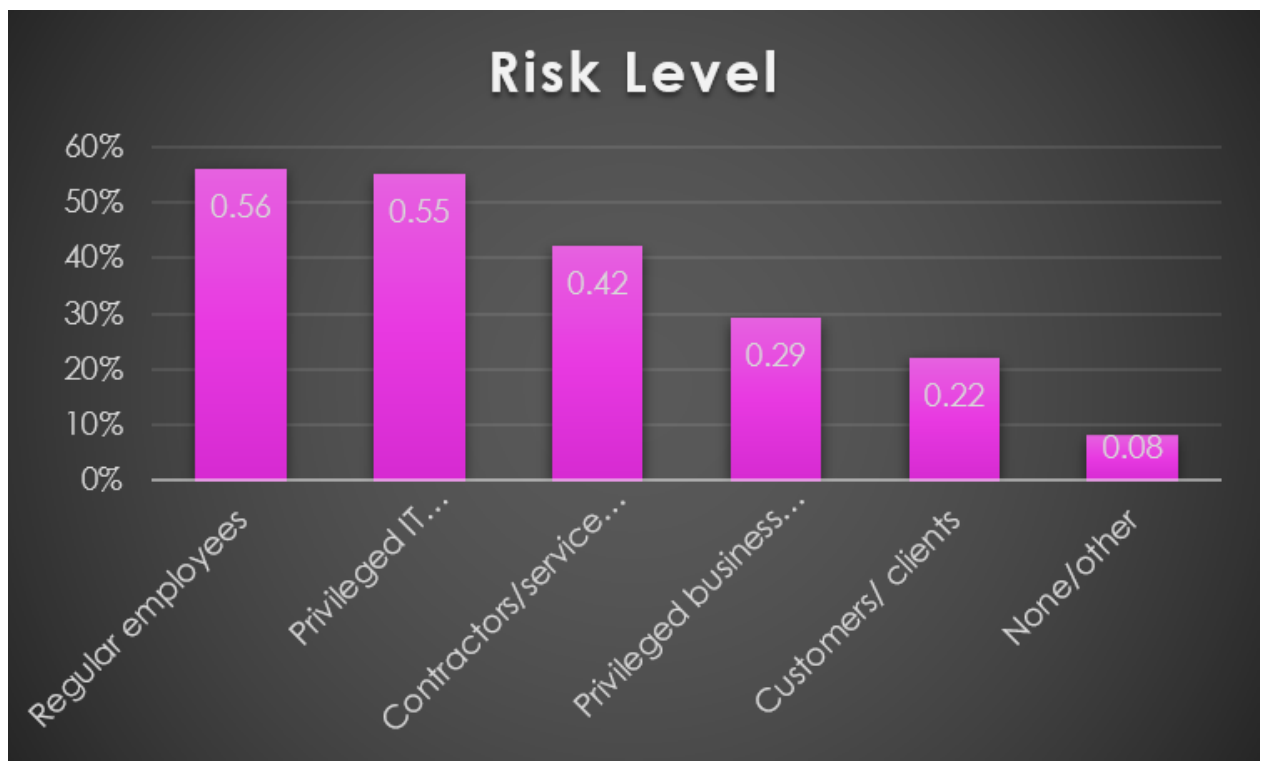

Figure 1.1: Types of insider threats and their risk level, from [14]

also shows users' unsafe decisions are the cause of $51 \%$ of the accidental/unintentional data breaches [14]. Users' decisions are shaped by their mental models [85], which act like filters affecting the way they see and interpret the world. When talking about security decisions, users' understanding of how things work would be of particular importance [12, 85, 86].

In the context of computer security and privacy, there is considerable literature on mental models. However, research on mental model related to data breaches is rare. This suggests the need to understand what people know and do not know about data breaches.

Researchers in the area of cybersecurity emphasize the role of security mechanisms such as firewalls, authentication mechanisms, virtual private networks (VPNs), and security technology like intrusion detection systems which are complementary to security mechanisms [57]. Despite the increased awareness of companies and great emphasis of security experts on security mechanisms, we see companies that implement poor security measures on data collection and data processing, therefore they will not be able to prevent compromises and information misuse. News headlines support this finding, and that major companies like Marriott and Equifax have exposed a massive number of consumers' records due to inadequate cybersecurity measures.

Data breaches happen due to companies' failure in protecting the data stored in 
their database servers. According to Coombs [21, the reputation of a company is based on the evaluation customers make about it. Customers' evaluation can be affected by the behavior of a company when a crisis like a data breach happens. So, due to the significant financial loss and reputational damage caused by data breaches, companies try to reduce the damages in the after-breach notifications using communication strategies [35, 83]. The crisis response strategies work as a framework to evaluate the reputational threats' factors and reduce their negative effects by changing the level of crisis responsibility. For example if a company frames the situation into a victim cluster or accident cluster, it will produce very little responsibility for the company [21]. We think that these strategies might affect users' perception of an incident, and also their mental models.

In crisis communication, the difference between incidents and crises reflects the severity of the impact of them. An incident is a minor disruption; however, a crisis disrupts the entire organization [21]. However, the word incident does not have the same connotation in the cybersecurity [82]. Therefore, we used "incident" whenever we wanted to refer to a data breach throughout this thesis.

\subsection{Research Goal}

The goal of our research is to explore users' understanding of data breaches; we are also interested to find out how breached companies and news media communicate with users, and how that might affect users' perception of a data breach incident.

\subsubsection{Research Questions}

1. What are peoples' mental models of an Internet data breach?

2. Are there differences among the breached companies' press releases and news when covering data breach incidents? and how might these strategies influence the public understanding of the data breaches?

\subsection{Contributions}

The contributions made by this thesis are as follows: 
1. We identified a basic model of data breaches from the literature. This model includes the taxonomy of breaches into insider and external threats, causes of a breach, how it happens, prevention methods, after breach steps, and the role of CERTs and CVEs.

2. We conducted study sessions with 20 participants to gain insights on people's mental models of Internet data breaches. We identified different facets of participants' perception of data breaches: what they are, how they happen, what are the cause, consequences, motivations, how it can be prevented and what can they do when their data is breached.

3. We analyzed 88 documents extracted from the companies' press release and reputable news related to Equifax and Marriott Hotels data breaches from September 2017 till March 2019. Findings of this study helped us to find the strategies used in the companies' notifications and their differences with the news media reports.

4. Based on the findings from study 1 and study 2, we conducted a quantitative study testing the mental models and the influence of companies' notifications and news on the general public.

\subsection{Thesis Outline}

This thesis has six chapters in total, this included; and they are structured as follows:

- Background. In this chapter, we provide an overview of related work about computer attacks and intrusion methods, intrusion detection systems, crisis communication in cybersecurity breaches, and users' mental model of privacy and security.

- Study 1: Exploring mental models of Internet data breaches. In this chapter, we present the study about the participants' mental model and reflect our findings. 
- Study 2: Crisis communication Analysis. The study of document analysis of Equifax and Marriott Hotels data breaches, and our findings are presented in this chapter.

- Study 3: Quantitative study on Internet data breaches. In this chapter, We presented our quantitative study of users mental model of data breaches and the influence of different documents on the users' perception, and our reflect our findings.

- Conclusion. In this chapter, we conclude our thesis and include a summary of our key findings from the three studies. We discuss the limitation of our work, and future research directions. 


\section{Chapter 2}

\section{Background}

A data breach is an incident in which a cybercriminal infiltrates a data source and extracts sensitive personal information such as an individual's name, social security number, driver's license number, financial record, et cetra [2]. All cybersecurity breaches usually contain personally identifiable information (PII) not in an encrypted format, which makes it easier to be read by attackers. PII is a term used to refer to the information that can be helpful to distinguish an individual's identity.

In this chapter, we review the literature relating to computer attacks and intrusion methods, and intrusion detection systems to review how the companies can defend themselves against a data breach before it happens. We also reviewed the literature on the crisis communication and cybersecurity breaches, and users' mental models of privacy and security.

\subsection{Overview of Computer Attacks and Intrusions}

A computer attack is any malicious and deliberate activity directed at a computer system or services to breach the information system. The possible types of computer attacks could be broken down into broad types: attacks that deny the authorized users access to the resources or services, attacks that probe a system to find vulnerabilities, attacks that include any executable malicious scripts, or attacks that allow intruders to gain privileged access or elevate his/her privileged access [46].

There have been numerous works attempt to categorize computer attacks. Hansman [30] provided a four dimension taxonomy based on the attack vector and the main behavior of the attack, the attack targets, vulnerabilities and payload. Alessandri [7] classified attacks into five elements that a given Intrusion Detection System design can detect, the characteristics of his classification includes: interface objects, affected objects, communication manners, call manners and other trivial attributions. Carver [16] suggested six categories of computer attacks based on time of attack, type 
of attack, type of attacker, credibility of attack, harm of attack and the prevention methods.

Ahmad et al. [47] divided Internet attacks into two subsections: Passive attacks, where the attackers aim to obtain the information, and Active attacks, where attackers make some modification in the original information or create some false message. Cisco [1] used thirteen categories for classifying the attack methods: sniffing, IP spoofing, right escalating, DoS/DDoS, cipher cracking, Man in the Middle attacks, application layer attacks, network probes, trusted relationship cracking, port redirection, unauthorized access, and viruses/worms/Trojans. These categories are not disjoint.

Wu et al. [88, classified attacks into seven main categories based on automated intrusion responses: Infection, Exploding, Probes, Cheats, Traverses, Concurrency and Others. In the Infection category, the attacker makes changes in the target system like installing an malicious file in it. The Exploding category contains Buffer overflow that happens when a buffer holds specific instructions for actions intended by a hacker or malicious user. The Probe category is where the attacker tries to collect information about the target system using tools. The cheat category containing IP Spoofing, MAC Spoofing, Session Hijacking, XSS Attacks, Hidden Area Operations, and Input Parameter Cheating is where attacker uses fake or abnormal input to deceive the victim. The characteristic of the Traverse category is to crack the system through simple matching of each possible key. It contains Brute Force, Dictionary Attacks and Doorknob Attacks. Brute force attacks involve repetitive attempts of trying different password combinations to break into a system. A Dictionary attack is a form of brute force, it happens by trying word lists in combination with common password lists to defeat authentication mechanisms; and Doorknob attacks happen when attackers search for vulnerabilities in computers that are connected to the Internet. The Concurrency category floods systems or services with traffic to exhaust resources, so that the system is unable to fulfill the legitimate requests. All others that can not be in the former categories, are classified as Others category.

Lazerevic et al. [57] provided a more general taxonomy based on the common characteristics among the existing categorizations and taxonomies of network attacks. They divide the computer attacks into the following classes: 
- Denial of Service (DoS) attacks. There are two subcategories of DoS: operating system attacks, where attackers target specific operating systems, and networking attacks, where attackers take advantage of a vulnerability in the networking protocols and infrastructures.

- Probing (surveillance, scanning). These attacks scan the network to collect information about valid IP addresses and usually find the vulnerabilities that can be used to attacked those systems.

- Compromises. Attackers use system vulnerabilities or weakness to break into the system and gain privileged access to hosts.

- Viruses/Worms/Trojan horses are computer programs that produce copies of themselves and propagate through a network.

- Viruses are programs that can replicate themselves by modifying other computer programs and are designed to cause considerable damage.

- Worms replicate themselves in order to spread through a network. Some of new version of worms have been used to launch DoS attacks like erkms and lion [51].

- Trojan horses are malicious programs that mislead the user of their true purpose.

All computer attacks will not necessarily lead to a data breach. However, attackers may use probing to find vulnerabilities in a system and compromises to exploit the systems' vulnerabilities that allow the attackers to get access to the companies servers and expose their data. Attackers can also use targeted malware attacks to get access to a target system.

\subsection{Intrusion Detection Systems (IDSs)}

Intrusion detection systems monitor a network or systems for malicious activity. Generally they will notify an administrator of a possible intrusion or collect centrally the malicious activity using a security information and an event management system. Intrusion detection systems are deployed to defend the companies from the data 
breaches and attacks that arises on a daily basis. Below we review some classifications of intrusion detection methods.

\subsubsection{Taxonomy of Intrusion Detection Systems}

Different classifications have been proposed in the literature for intrusion detection methods. Debar et al. 32] used the following concepts to classify intrusion detection systems: detection method, behavior on detection, audit source location, usage frequency. Detection methods describe the characteristics of the analyzer which could be knowledge-based or behavior-based. Knowledge-based is when the IDS looks for events that match a known attack and behavior-based is when the IDS looks for unusual behavior that differs from the normal behavior of the system. The intrusion detection system's response to an attack can be described by behavior on detection, and audit source location describes possible sources of data an intrusion detection system can use. It can be audit trails, system logs or network packets. Usage frequency is based on if the intrusion detection system have real-time continuous monitoring capabilities or it must be run periodically.

Lazerevic et al. [57] presented a taxonomy based on the synthesis of a number of existing classifications (see Figure 2.1.

There are many possible source of data that can be provided to an IDS, like audit trails on a host, network connections/packets, application logs, wireless network traffic, or sensor alerts produced by other IDSs, so information source is one of the criteria for classification of IDSs. The analysis strategy involves the same criteria as detection methods described by Debar et al. 32]. the time aspect in this taxonomy was defined as usage frequency. An IDS can have a centralized architecture that analyzed the data from one monitored system or a distributed architecture that collect data from multiple monitored systems. If an IDS responds actively, it takes corrective action or pro-active action to an attack, and if an IDS responds passively, it only generates alarms and does not take any action.

\subsubsection{Classification of Detection}

There are three approaches for detecting attacks: misuse detection, anomaly detection [57], and Hybrid-based [75]. Misuse detection is based on the knowledge of known 


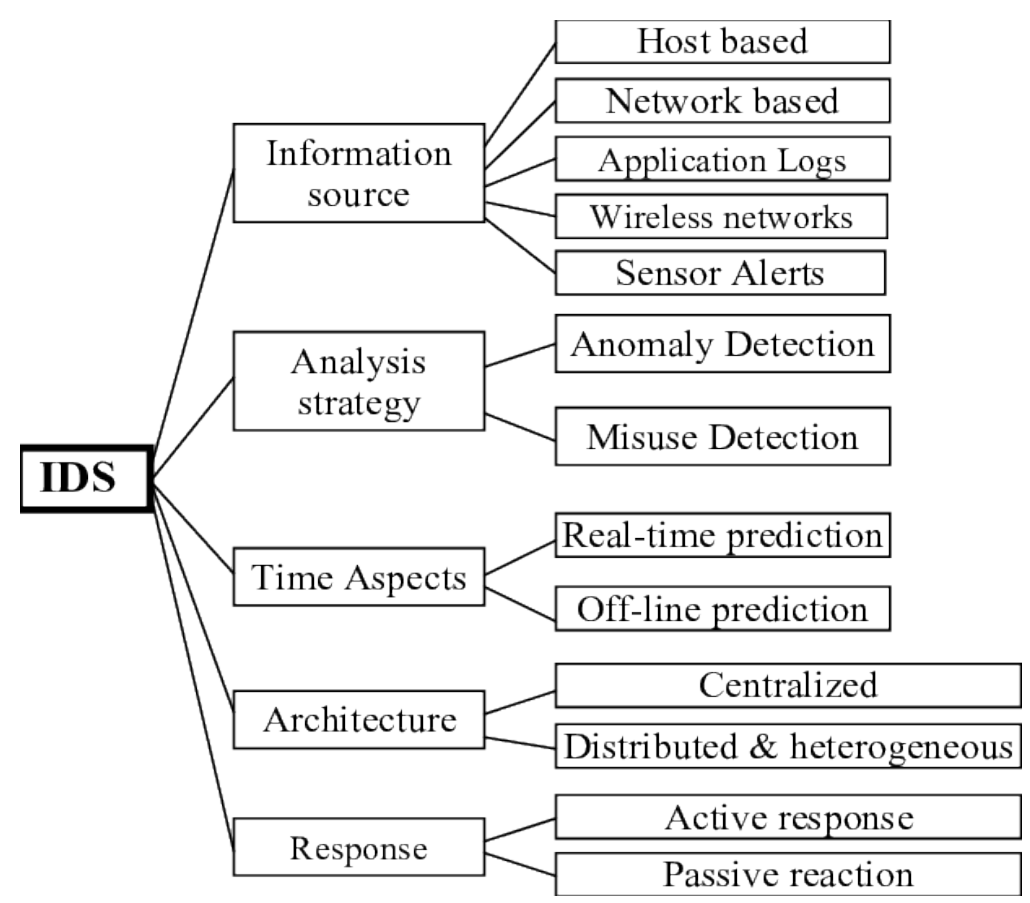

Figure 2.1: Taxonomy of intrusion detection systems from Lazerevic et al. [57]

attacks and system vulnerabilities. In misuse detection, the system looks for attackers to perform known attacks or to exploit known vulnerabilities. It is very accurate for known attacks, however, it is not a suitable approach for unknown ones. Misuse detection approach can be classified into the following groups:

- Signature-based techniques: They are used to search for known identities in a database of signatures for each intrusion detection event. In this approach the system looks for patterns or clues that are thought to be the evidence of intrusive activity, no matter what constitutes the normal behavior of the system.

- Rule-based systems: In this technique, a set of "if-then" rules is used to determine computer attacks.

- State transition analysis: In this technique, it is first needed to construct a finite state machine, in which states correspond to different IDS states and then characterize events that cause changes in IDS states.

- Data mining based techniques: In this method, each instance in the data set is labeled as 'normal' or 'intrusive' and then we train a learning algorithm over the labeled data to detect non-normal activity. 
In the anomaly detection approach, we do not look for known intrusions but rather for unforeseen and emerging cyber activities, and patterns in network traffic that are different from legitimate activities. Axelsson [9] divides the anomaly detection approach into two classes: self-learning systems, where systems observe traffic for a period of time and then build a model of the underlying process, and programmed class, where the system needs to be taught or to be programmed to detect anomalies. Lazerevic et al. [57] classified the anomaly detection techniques into five groups based on employed techniques: statistical methods, rule based methods, distance based methods, profiling methods, and model based approaches.

In the statistical methods, they observe the system behavior and measure certain variables over time. The primary version of this model keeps the averages of variables and compute the standard deviation of them and detect if it is changing or not. Distance based approaches can be used to find global and local anomalies [13, 56] by computing distances of points from one another [52]. In rule based systems, we characterize the normal behavior of the system by a set of rules. Profiling methods are based on building profiles of different types of network traffic, users, programs and etc. and intrusion will be detected if there is any deviation from them. In model based approaches, researchers create models of normal behavior and any deviation from those models counts as intrusion.

\subsection{Anatomy of a Cyber Security Breach}

In this section, we present a basic model of a data breach; containing information about the causes, how it happens, CERTs and CVEs, and what the companies as well as customers should do.

A data breach is the intentional or unintentional release of sensitive information to unauthorized parties. The type of Personally Identifiable Information (PII) that cybercriminals can gain access through data breach might include following: Username and passwords for online accounts, payment cards and other financial information, passport number, bills, contracts and other important documents, postal address,

email contacts and phone number, emails and instant messages, sensitive photos and other data that can be used for blackmail, system configuration. 


\subsubsection{What cause an Internet data breach?}

One approach to categorize data breaches is based on the parties who caused the breach: insider or outsider threats [53]. Insider threats are normally caused by the following main types:

- Employee Error/Negligence: people make mistakes all of the time, so when it comes to data breaches, something as simple as sharing something unintentionally in publicly accessible locations like social networks can cause a breach.

- Lost or stolen devices: Company devices that contain confidential information may be stolen or lost.

- Email, Webmail, and removable devices: An employee may copy confidential data to a USB device or email it to a home email account without proper encryption. So, in this case, data can be exposed to attack during the transmission or on the unprotected home devices [3].

- Cyber espionage/ sabotage: A company's breach can be caused by an employee deliberate actions like espionage for financial reward or employee grievances.

- Unauthorized Access: This form of a data breach could happen due to a lack of access controls by a system admin.

The possible causes of external data breaches include the following types:

- Hacker break-ins: The attackers may exploit system vulnerabilities to gain access to a corporate network.

- Malware: The attackers, disguised as a known entity, use spam, email or instant communication to direct the user to a compromised website with malware and provide the attacker control of the user's computer to access the confidential information.

- Social engineering: Social engineering attacks are used against enterprises to be deceiving employees and individuals into disclosing confidential or personal information to cybercriminals [33]. 


\subsubsection{What happens in an Internet data breach?}

Attackers identify first a target based on the motive of causing harm or damage or having a profit from the breach. Then they follow the following steps to get access to the confidential information [79, 33, 3]:

- Search: The attacker looks for vulnerabilities in the target's people system or network. Scanning programs can be used to determine the location of data repositories or scan internet addresses across the internet to see if the potential targets are using any version of the software that is vulnerable to attack.

- Attack: The attacker breaks into the targets' system through a network attack or social attack. In a network attack, the attacker uses the weakness in a network using techniques like SQL injection, targeted malware, session hijacking, etc. In a social attack, the attacker uses social engineering tactics that can begin with a phishing email with malware, among others.

- Discovery: The attacker automatically scans the system for confidential data.

- Capture: Data in unprotected systems is immediately accessed, and the attacker can also install root kits (software that enables an unauthorized user to get access to a computer system without being detected) on the targeted systems and network access points to capture more data.

- Exfiltration: The attacker executes data queries to transfer data to the hacker team; they may take steps to remove the information from the company's databases.

\subsubsection{CERT \& CVE}

The first Computer Emergency Response Team Coordination Center (CERT/CC) was created at Carnegie Mellon University's Software Engineering Institute in the late 1980's. The CERT/CC was chartered to respond to Internet security events. Today there are several Computer Security Incident Response Teams (CSIRTs) serving a variety of organizations. They are focused on the prevention of, handling, and response to cybersecurity incidents [48]. 
Since cyber security incidents usually cross many borders, creation of national CSIRTs is represented as the only way to prevent and resolve widened cyber security incidents. To be effective in collaborating globally, national CSIRTs need to achieve a level of maturity [74.

Common Vulnerabilities and Exposures (CVE) databases are security information sources that provides a list of publicly known cybersecurity vulnerabilities. CVEs are an international community effort; numerous organizations around the world have adopted the use of CVEs, and they also have made their products and services compatible with CVEs [84].

\subsubsection{What should companies do?}

It is crucial for companies to take steps to prevent a data breach. The following practices will increase the network's defense [33]:

- Patch and update of all the software and hardware.

- Educate employees about social engineering tactics.

- Implement and update security measures.

- Use intrusion detection systems.

- Employ multilayered protection: antivirus software, data access control, encryption, and strong policy enforcement.

- Be aware of CERTs announcement of new vulnerabilities, and check the CVEs regularly.

- Evaluate the impact of personal data processing by conducting relevant assessments as advised by GDPR [20].

- Be ready to notify data breach victims, including time frames, methods, and parties that should be informed when PII is compromised [78]. 


\subsubsection{What should the customers do?}

Consumers who are at risk of being affected by a data breach should know how they can protect themselves. The following steps can help them to prevent a data breach as an individual [33]:

- Keep themselves aware of the new regulations, and expect the companies to provide secure data processing and data retention as advised by GDPR [20].

- Regularly check their banking transaction receipts.

- Use security software such as antimalware with a personal firewall installed on their system.

- Use multi-factor authentication.

- Make sure that all of their software is updated with the latest security patches.

- Create a strong password and use a different combination of user and passwords for different accounts.

- Be aware of phishing emails and do not click links in or open files attached to that when they doubt about the legitimacy of their senders.

- Keep themselves aware of the threats that are existing so they can protect themselves accordingly.

\subsection{Data Breaches and Crisis Response}

Data breaches are a severe threat to both organizations and consumers, and are increasingly common events across businesses. The loss of sensitive information can lead to financial losses and significant reputational damage for enterprises and the increased possibility of identity theft, credit card fraud, financial loss for consumers.

When a data breach happens, the enterprise facing this crisis should inform the legal authorities, and it also needs to notify all the affected and potentially affected consumers. According to Kelly [45], the company's communication during a crisis can lead to negative media attention and thus ruin the company's image and result 
in the loss of consumers' trust. Therefore, it is crucial to know how and what an organization communicates with the consumers during a data breach.

Strategic crisis communication researchers focus only on how crisis communication can be used to achieve the best outcome for the organizations and reduce the adverse impact of breaches on companies' performance, but there was less information on reduce damage, establish accountability, and understand the cause and effect of a data breach. We think that is due to the mission of crisis response strategies which is a framework for companies to evaluate their reputational threats' factors and reduce it so that customers evaluate the corporate as trustworthy and reliable.

Sturges [54] categorize crisis responses into three strategic focus:

- Instructing information, managing the crisis physically;

- Adjusting information, managing the crisis psychologically;

- Reputation repair, correcting the damage a crisis imposes on an enterprise.

The informal crisis communication research is focused on three schools of thought: corporate apologia, image restoration, and renewal [21].

The concept of apologia is used for self-defense and was applied for the first time to corporate communication by Dionisopolous [24]. The common components of an apology are responsibility admittance, sympathetic expression, reassurance, and compensation [59]. When an enterprise apologizes, it means that the enterprise accepts its responsibility even if there is no explicit statement of that. To show sympathy, the corporations need to express an understanding and concern for the people involved in the crisis. Reassurance is when the enterprise try to convince the readers that the same negative event will not happen again [58].

Image repair theory is the most powerful framework, presented by William Benoit [10] that can be used by practitioners to design messages during a crisis, and be used by critics to evaluate the created messages. The key concept in this theory is to understand the nature of complaint or attack. An attack has two components: an offensive act, and the accusation of responsibility for an action. According to Benoit, the image repair strategies can be categorized into three sub-categories: denial, evasion of responsibility, and reducing the offensiveness of event. Denial is a general approach to image repair, and it is about rejecting responsibility. Evasion of responsibility 
contains four sub-strategies: provocation (response to someone else's action), defeasibility (lack of information or control over the situation), accident (did not mean it to happen), and good intention. Reducing the offensiveness of an event also involves a list of potential response strategies that are as following: bolstering (reminds of good traits), minimization (claim that act was not serious), differentiation (reduce offensiveness of the act), transcendence (place the act in more favorable context), attack accuser (challenge the credibility of accuser), compensation (reimburse for the act), corrective action (restore situation or promise that the act will not happen again), and mortification (ask for forgiveness). Renewal is the most recent informal crisis communication, and it emphasizes a positive view of the future [21].

Data breach notifications are a form of negative news messages because they go further than correspondence or routine messages, and they can be a public relation nightmare. There are two main organizational patterns for a negative message: indirect and direct. In the literature, authors suggest communicating with consumers using the indirect pattern [34]. They argue that when a problem is significant, hiding the bad news within the explanation or using passive voice can reduce the shock and sadness of the readers [62, 34]. However, Veltsos [83] shows that most of data breach notification templates follow the direct approach. She did a qualitative analysis of 13 data breach notification templates. She found that data breach notifications follow standard business letter formatting for negative messages. Since the purpose of a data breach notification is to convince the consumers that a potential risk exists and to encourage them to act, the direct pattern of document design is more helpful. According to the direct pattern, writers need to present the bad news in the first part of the message and then provide information about the protective actions.

Kim and Lee [50] did an empirical study on cybersecurity breaches in post- breach crisis communication. They compared 108 official statements in the United States and South Korea to investigate the characteristics of corporate apologies. They found that the features of the official statement differ by culture. They also found that communication style can be another factor that affects the official statement of data breaches.

Kim et al. [49] did a content analysis study of 64 news stories of five security breach crises. They analyzed press releases and news releases in four national online 
newspapers to explore crisis response strategies and framed situational factors. They found that there are dissimilar approaches used in press releases and news when reporting the crisis. Jenkins et al. [35] analyzed the effect of impression management techniques. They did two studies to examine the performance of language in the notifications sent to consumers affected in a data breach. In the first study, they used a content analysis methodology to explore a random sample of data breach notifications, where data was coded using speech act theory, felicity conditions, visual/ presentation effects emphasis. In the second study, they measured the impact of impression management practices on affected consumers in the data breaches. The results of these two studies demonstrated that using impression management tactics can produce a positive outcome in post-breach communication.

Cross et al. 222 studied a particular case involving a data breach, where the information of the users of an online dating website were exposed, the website was known for facilitating extramarital affairs. Because of the character of the site, the nature of the data exposed was more sensitive. This data breach had a severe personal impact on victims. The authors were interested to see how this incident was reported in the news media. They found that because of the perceived immorality of the website, instead of focusing on the data breach and the criminals who exposed the information, the news kept blaming the victims.

All in all, the literature in data breach crisis communication demonstrate that crisis communication theories are focused on the evaluation of the situation in favor of enterprises to be prepared and recover from a crisis. Depending on the context of a data breach companies and news strategies can be different reporting the same incident.

As the number of cybersecurity breaches is increasing, agencies like the U.S. Federal Trade Commission (FTC), that has a mission of protecting consumers, prepared a guide for companies for a data breach response [19]. A breached company has to notify law enforcement, affected businesses and individuals. Individual notifications should contain information about state laws, the nature of the compromise, the type of information taken, the likelihood of misuse, the potential damage if the information is misused. The companies need to clearly describe what they know about the breach including: 
- How it happened;

- What information was taken;

- How the attackers have used the information;

- What actions the company has taken to remedy the situation;

- What actions the company is taking to protect individuals, such as offering free credit monitoring services;

- How to reach the relevant contacts in the company.

Depending on the type of information that was disclosed, they may need to give the consumers some information about appropriate follow-up steps, how to recover from identity theft, and how they will contact consumers in the future.

\subsubsection{Narrative-semiotic approach}

Semiotics means the science of signs, and semioticians believe that communication is symbolic and ambiguous, and it happens through perceptual or linguistics signs between interlocutors. Narrative-semiotics is a method of text and discourse analysis that provides a valuable, comprehensive, overview for analyzing crisis communications in professional contexts [64]. This method compares the structure of an organization parallel to the construction of a story. We can divide the Narrative-semiotic approach into two aspects: the narrative trajectory and the narrative schema [66].

The narrative trajectory is the sequences of events and actions that create a story; it has the following stages:

- An initial situation, which states the normal state before any events happen.

- A complication, which includes any challenges or accidents that cause problems for the organization.

- Actions taken to address the complication, which is the companies strategies to resolve the issue.

- Resolution, which represents the end of the story, either solved or compromise reached. 
The narrative schema consists of the six categories of agents known as the actantial model. The actantial model, developed by A.J.Greimas [31], can be used to break an action down into six positions:

1. The sender category includes agents who direct the action of subject towards an object.

2. The subject category consists of the leading performers and is what is directed towards an object.

3. The object category includes the desired outcomes and objectives.

4. The receiver category consists of the agents who benefits if a desired goal is achieved.

5. The helper category includes agents who assist in achieving the desired goal like experts who aid the subject in reaching the goal.

6. The opponent category includes agents who hinder the achievement of the desired goal like lack of knowledge or ability, and ineffective tools.

Narrative-semiotics can be used to identify the agents, their actions, and their discourses. Different actions reflect different points of view, that is why it is vital to compare different texts on the same subject. A series of events can be told differently when the narrator changes. Narrators position the agents through the way they tell the story. So the narrator has a pivotal role in identifying a narrative configuration which can be used to see how different narrator interpret the actions and highlight the patterns in their interaction [64]. Comparing the narrative configuration of different texts can reveal how companies narrate a crisis like a data breach and how it can be different from the news.

\subsection{Users' Mental Model of Privacy and Security}

Cybersecurity issues are becoming more common. Recent research suggests that people and companies have poor cybersecurity practices [4]. Things change quickly in the world of cybersecurity, and new threats appear every day. Human factors like a user's online decisions can result in computer security threats. Users' decisions are 
shaped by their mental models [71], which act like filters affecting the way they see and interpret the world. When talking about security decisions, users understanding of how things work would be of particular importance [12, 85, 86]. In other words, security will be understood using mental models.

Mental models are a common method in psychology to capture users' understanding of a problem [36]. A mental model "is based on belief, not facts: that is, it is a model of what users know (or think they know) about a system" [72]. Since mental models show natural ways of user interactions with a system, they can be useful in educational materials.

Jean Camp [15] presented five possible metaphorical mental models for security failures: physical security, medical infection, criminal behavior, economics failure, and warfare. Physical security models are somewhat understood; for instance, the existence of a wall indicates a network security practice. This model can encourage users to secure their computers. The public health model reminds the ability of users to protect themselves, and it shows shared responsibility for community health. In the criminal behavior model, malicious behavior is a crime; then we will have victims who suffer a significant loss. So, law enforcement is a response, and increased surveillance is a prominent part of the solution to the computer crime's problem. The warfare model evokes the existence of a determined enemy and the critical need for response and reminds the users that individual actions are crucial for collective security. Computer security failures can be seen as an economic failure; IT services to address the bad security behavior also cost for an organization. So, this model can be used to convince the users that they have valuable assets and any thief might target them. Each of these models implies a different solution, and therefore, when communicating with users, computer security experts should consider which model will meet the user expectations.

Other studies on users' mental models in computer security were focused on users' understanding of security issues using methods such as surveys, and observation of how people interact with the system. There are different methods in the literature used by researchers to assess mental models. These methods are as follows: problem solving, verbal report, drawing, categorization, and conceptual pattern representation. Based on the problem solving method, people use their mental models 
to understand a problem and making inference and prediction [37], so people with different mental models will understand a problem differently and will come up with different solutions. A verbal report is a direct method of eliciting mental models and can be done as interviews, explanation or think-aloud protocols [17]. The categorization method shows how mental models are developed and categorized, it usually has been used to show the similarities and differences between experts and novices [18]. Mental models can also be represented in the form of concept maps which represent the concepts and their relationships [41, 40]. Diagramming tasks are another method which is considered as a way of capturing mental models in addition to traditional verbal reports [38] and this method is mostly used in user-centered research. For think-aloud, participants of a study are asked to verbalize their thoughts while performing a task. Think-aloud is useful for analyzing mental models because it provides direct information about the participants' thinking process. Since mental models are usually pictorial, using verbal reports have some limitations [39]. For this reason, drawings and verbal reports have been used as complementary methods in several mental model research studies. For example, Raja et al. [76] conducted a study on participants' mental models of the Vista Firewall using a diagramming task. They gave participants a picture of a computer, a firewall, and the Internet cloud and asked them to show how the Vista Firewall works by drawing arrows. Finally, they designed a new interface with contextual information to improve participants' mental model of the firewall.

Asgharpour et al. [8] found a remarkable difference in the mental models of security experts and non-experts who have lower security literacy. Their results show that the domain of expertise has an impact on user behavior. They suggest that the design of risk communication should be based on non-experts' mental models. Wash [85] used multiple rounds of interviews to understand users mental models of security threat. He identified eight folk models of malware and hackers that break into victims' computers; users used this models to make a decision for which security software to use and which advice to follow.

Friedman et al. [28] also analyzed web users concerns about security risks, interviewing seventy-two individuals asking them some standardized questions to evaluate 
their understanding of web security. They found that people with different educational background could have different concerns, but they most often emphasized security threats and privacy. Wu and Zappala [87] conducted a series of semi-structured phone interviews using both interview techniques and diagramming exercises to capture users perception of the encryption process. They found four mental models which are different in details and complexity. They also found that users do not perceive a decisive role for encryption in daily life. Kang et al. [43] focus on what people know and do not know about the Internet and how that knowledge affects their reaction to privacy and security risks. They found that lay people have less sophisticated mental models related to computer security. To protect users security and privacy, they suggest not to rely too much on users' security practices.

Understanding mental models of the end-user can lead to better communication regarding privacy and security risks [8]. Having poor mental models of data breaches can be echoed in behavior, and people will underestimate the consequences and risks of a data breach when they are not aware of that. Attackers exploit human errors as well as technology failure to get access to confidential information. Therefore, the human element is a key and user's lack of knowledge and awareness is a cause in a cybersecurity breach, something as simple as clicking on a link in the email or sharing something unintentionally in public can lead to a massive breach of data. Users need to know: what is a data breach? How do data breaches happen? What are the consequences of a data breach? How do individuals and companies prevent data breaches? What are the companies responsibilities regarding securing people's personal data and what do they require to do after a data breach? As they perceive the risk of a data breach, they will be more concerned about the consequences, will take protective measures seriously and they will understand what should they expect from the breached companies when they are affected by data breaches. 


\section{Chapter 3}

\section{Study 1: Exploring Mental Models of Internet Data Breaches}

In our background section, we presented a basic model of an Internet data breach (see section 2.3. In this study, we wanted to explore users understanding of Internet data breaches that would endanger the user's Personally Identifiable Information (PII), and see if users know the basic information that we provided in our background chapter. To this end, we conducted a study in which we asked users to describe and diagram their understanding of how a data breach happens. We collected data about participants' perception of Internet data breaches, their perception of the causes, motivations, consequences, and their attitudes towards preventing a data breach.

\subsection{Methodology}

The data presented in this work is from 20 one-on-one in-person sessions with participants about their mental models of Internet data breaches. Each session lasted 40 to 90 minutes, and we reimbursed them $\$ 20$ for their time. Our study was reviewed and cleared by Carleton University Research Ethics Board (Clearance \#110780).

We collected qualitative and quantitative data through a drawing task, as well as open-ended and close-ended questions administered through LimeSurvey. The researcher audio-recorded the participants' voice while they were verbally explaining their understanding of Internet data breaches, these recordings were transcribed, and reviewed during the analysis.

\subsubsection{Session}

The sessions took place in Carleton's HotSoft lab or off-site. Sessions lasted between 40 to 90 minutes, depending on how much time each participant took in doing the drawing task and answering the questionnaires. Participants read and signed a consent form, the researcher explained the tasks they would be completing during the session, and they proceeded to participate in the following: 
1. Demographic and SeBIS questions: This questionnaire was administered on a provided computer using LimeSurvey (an open source survey system, hosted on our secure server). Besides basic demographic questions, the demographic questionnaire included two questions about whether participants have experienced any data security problem and identity theft. They also responded to the SeBIS (Security Behavior Intentions Scale) questionnaire, which include 16-item scale questions for gauging the participants' attitudes towards choosing passwords, device securement, staying up-to-date, and proactive awareness [25] (see Table 3.1).

2. Drawing task: Participants were first informed that the purpose of this exercise was to help the researcher to understand what the participant had in their mind, and it was not a test of artistic ability, and there was no correct way of drawing the picture. They were asked to draw their understanding of how a data breach happens. The drawing prompt was: "Using a paper and pencil, please draw your understanding of how a data breach happens. In other words, try to draw what is happening when it is said that personal information was exposed in a hack. (Elements you may need for this drawing: Admin, Data, Customer, Hacker, Access to data, Vulnerability). Each participant was given as much time as they needed to finish the drawing task. We used a think-aloud protocol; the participants were told to articulate their understanding of how a data breach happens while working on the drawing task. Participants were audio-recorded during the diagramming exercise using the Windows Voice Recorder app, and then the picture of the drawing was scanned and uploaded on LimeSurvey.

3. Post-test questionnaire: This questionnaire was administered in the same way as the Demographic and SeBIS questions. Participants answered questions about whether they have ever heard of an Internet data breach, what is considered an Internet data breach, what are the causes, consequences, motivations, and prevention methods for a data breach (see Table 3.2). Finally, the last segment of the session involved answering the Likert-type questions on a 5-point scale where participants choose the likelihood of causes, consequences, motivations, and prevention methods of a data breach. See Appendix for the complete 
Table 3.1: SeBIS questions [25]. Responses were reported on the following scale: Never (1), Rarely (2), Sometimes (3), Often (4), and Always (5)

\begin{tabular}{|l|l|}
\hline$\#$ & Device securement \\
\hline 1. & $\begin{array}{l}\text { I set my computer screen to automatically lock if I don't use it for a prolonged } \\
\text { period of time. }\end{array}$ \\
\hline 2. & I use a password/passcode to unlock my laptop or tablet. \\
\hline 3. & I manually lock my computer screen when I step away from it. \\
\hline 4. & I use a PIN or passcode to unlock my mobile phone. \\
\hline \hline$\#$ & Password generation \\
\hline 5. & I do not change my passwords, unless I have to. \\
\hline 6. & I use different passwords for different accounts that I have. \\
\hline 7. & $\begin{array}{l}\text { When I create a new online account, I try to use a password that goes beyond } \\
\text { the site's minimum requirements. }\end{array}$ \\
\hline 8. & I do not include special characters in my password if it's not required. \\
\hline \hline$\#$ & Proactive awareness \\
\hline 9. & When someone sends me a link, I open it without first verifying where it goes. \\
\hline 10. & $\begin{array}{l}\text { I know what website I'm visiting based on its look and feel, rather than by } \\
\text { looking at the URL bar. }\end{array}$ \\
\hline 11. & $\begin{array}{l}\text { I submit information to websites without first verifying that it will be sent } \\
\text { securely (e.g., SSL, "https://", a lock icon). }\end{array}$ \\
\hline 12. & $\begin{array}{l}\text { When browsing websites, I mouseover links to see where they go, before clicking } \\
\text { them. }\end{array}$ \\
\hline 13. & $\begin{array}{l}\text { If I discover a security problem, I continue what I was doing because I assume } \\
\text { someone else will fix it. }\end{array}$ \\
\hline \hline$\#$ & Updating \\
\hline 14. & When I'm prompted about a software update, I install it right away. \\
\hline 15. & I try to make sure that the programs I use are up to date. \\
\hline 16. & I verify that my anti-virus software has been regularly updating itself. \\
\hline
\end{tabular}




\begin{tabular}{|l|l|}
\hline$\#$ & Questions \\
\hline 1. & What is considered an Internet data breach, as you understand it? \\
\hline 2. & $\begin{array}{c}\text { Have you ever heard of an Internet data breach? } \\
\text { If you answered yes, which one do you remember? where did you read } \\
\text { about that? }\end{array}$ \\
\hline 3. & What causes an Internet data breach? \\
\hline 4. & What happens in an Internet data breach? \\
\hline 5. & $\begin{array}{l}\text { Why does Internet data breach happen? In other words, what purpose is } \\
\text { behind an Internet data breach? }\end{array}$ \\
\hline 6. & How would you know if you have been affected in an Internet data breach? \\
\hline 7. & What can you do if your data has been breached? \\
\hline 8. & What should companies do to protect your data from data breaches? \\
\hline 9. & How can Internet data breaches be prevented? \\
\hline 10. & What are the consequences of Internet data breach? \\
\hline 11. & Who is responsible for data protection? \\
\hline
\end{tabular}

Table 3.2: Open-ended questions from the post-test questionnaire

questionnaires.

\subsubsection{Participants}

Participants were recruited using recruitment posters placed on billboards at Carleton University and advertising the study poster on Carleton's Facebook page for user study recruitment; known contacts were also emailed. We recruited 20 participants; 10 female and 10 male. They ranged in age from 20 to 54 years. A list of all the participants is shown in Table 3.3 . When asked specifically about if they have ever experienced any data security problem, seven said yes, eight said no, and the remaining five said that they were uncertain about it. When asked if they have ever experienced identity theft, fifteen said no, three said yes, and the remaining two said that they were uncertain about it. Nineteen participants accepted to be audiorecorded, and only one participants did not accept to be audio-recorded, so we took notes during that session. 
Table 3.3: Study participants (Total=20)

\begin{tabular}{lllll}
\hline ID & Gender & Age & Education background & Academic level \\
\hline \hline P1 & F & 26 & Biology/Math/Education & Master \\
P2 & F & 27 & Physical Education/Education & Bachelor degree \\
P3 & M & 27 & Accounting & Bachelor degree \\
P4 & M & 21 & Neuroscience/Biology & Bachelor degree \\
P5 & M & 20 & Electrical Engineering & Bachelor degree \\
P6 & M & 22 & Computer Science/Computer Security & Bachelor degree \\
P7 & F & 35 & Information Technology & Bachelor degree \\
P8 & F & 31 & TEFL & Master \\
P9 & M & 35 & Applied Linguistics & Master \\
P10 & M & 33 & Engineering & Master \\
P11 & M & 21 & Film Studies & Bachelor degree \\
P12 & F & 54 & Cognitive science & High school diploma \\
P13 & F & 37 & HCI/English literature & Master \\
P14 & M & 29 & Psychology & Ph.D. \\
P15 & F & 22 & Law and Legal Studies & Bachelor degree \\
P16 & F & 20 & Psychology & Bachelor degree \\
P17 & F & 26 & Healthcare & College degree \\
P18 & M & 38 & Engineering & Master \\
P19 & F & 28 & HCI & Master \\
P20 & M & 31 & Computer Science & Ph.D. \\
\hline
\end{tabular}




\subsection{Data Analysis}

We addressed participant responses in two sections: qualitative data, and quantitative data stemming from Likert Scale questions. We qualitatively analyzed participants' think aloud comments during their drawing task, their drawings and their responses to open-ended questions. We conducted a thematic analysis [29]. We started by focusing on the diagrams several times to understand the data as a group. We were looking for key elements, trends, themes, or ideas in the images that will help outline the analysis. We generated a set of codes by comparing the similarities and differences between the participants' drawings. The codes are representative of the identified themes and are linked to drawings as summary markers [42, 26]. We built an initial summary of these codes and we tried to identify patterns in the way participants talked about data breaches and their word choices, and we looked for the cases where they had different perceptions. The recording were transcribed using the Otter.ai. We used the transcription as a complementary method for the drawing task. The transcription helped the researcher to understand the participants' drawings better where it was not clear.

The high-level codes for open questions are associated with our research questions and they are focused on (1) what is considered a data breach; (2) causes of an Internet data breach; (3) reasons why an Internet data breach happens; (4) how to know if a user has been affected in an Internet data breach; (5) what are the consequences and prevention methods; and (6) who is responsible for data protection. These themes are top-level categories and we created several sub-categories based on participants' responses.

We analyzed the results of the SeBIS questionnaires by aggregating the score of positive questions minus the score of negative ones. Each of the questions were scored 1-5, where 1 stands for "Never", 5 for "Always", and 3 for "Sometimes". The result of the other closed questions was analyzed by computing the median for the central tendency of our data, as well as analyzing and reviewing graphical representations. 


\subsection{Results}

\subsubsection{SeBIS Questions Results}

The boxplots in Figure 3.1 shows a summary of the results of SeBIS questionnaire. The results of the SeBIS questionnaire illustrates the computer security behaviors of participants. In response to the first four questions about device securement, participants reported that they always lock their computer screens automatically, they use a password/passcode to unlock their laptop or tablet, and they use a PIN or passcode to unlock their mobile phone $(M=5 / 5)$. They often manually lock their computer screen when they step away from it. They report poor behavior regarding password generation: they never change their passwords unless they have to $(1.5 / 5)$, they sometimes use different passwords for different accounts, they sometimes use a password that goes beyond the site's minimum requirements, and they often include special characters in their password even if it's not required $(3 / 5)$.

When asked about proactive awareness, they reported that they sometimes open a link without first looking to see where it goes. They often look at the URL bar to check what website they are visiting, they sometimes submit information to websites without first verifying that it will be sent securely, and if they discover a security problem, they sometimes $(3 / 5)$ continue what they were doing because they assume someone else will fix it. They report neutral behavior regarding updating software and anti-virus (3/5). The histogram in Figure 3.2 shows their different level of expertise. The range of participants score could be between 0 to 80 and the probability densities shows that people are more likely to score 55 in their SeBIS questions.

\subsubsection{Questionnaire Results}

At the end of each session, participants were asked to answer another questionnaire consisting of some open-ended questions and four series of five-point Likert-scale questions which were grouped to represent the following categories: definition of a data breach, responsibility for data protection, causes, consequences, motivation, and prevention methods. In each of the closed questions, 1 stands for "very unlikely" or "strongly disagree", 5 for "very likely" or "strongly agree", and 3 for "neutral" or "undecided". All the reported number are the calculated median. 


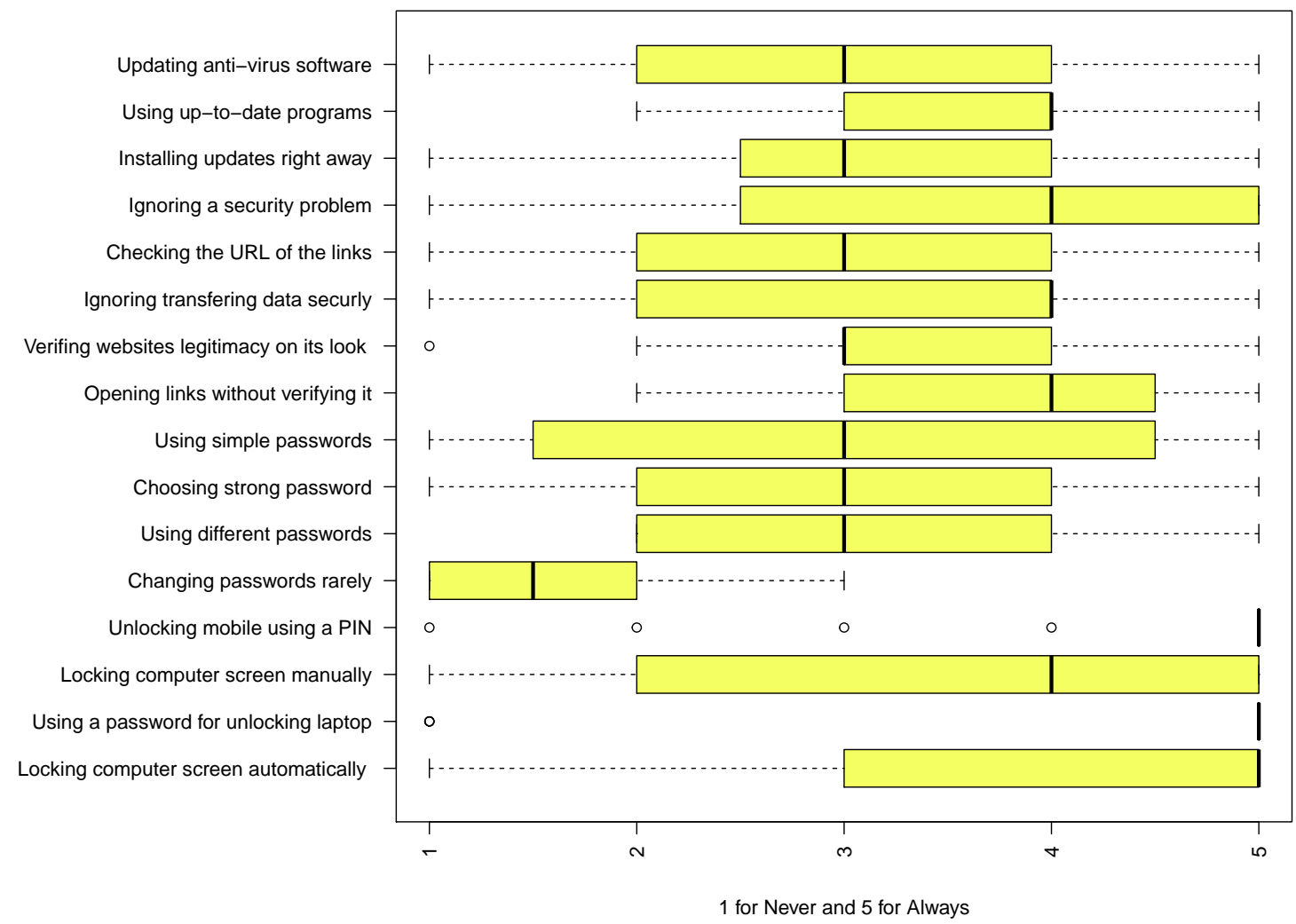

Figure 3.1: SeBIS questions 


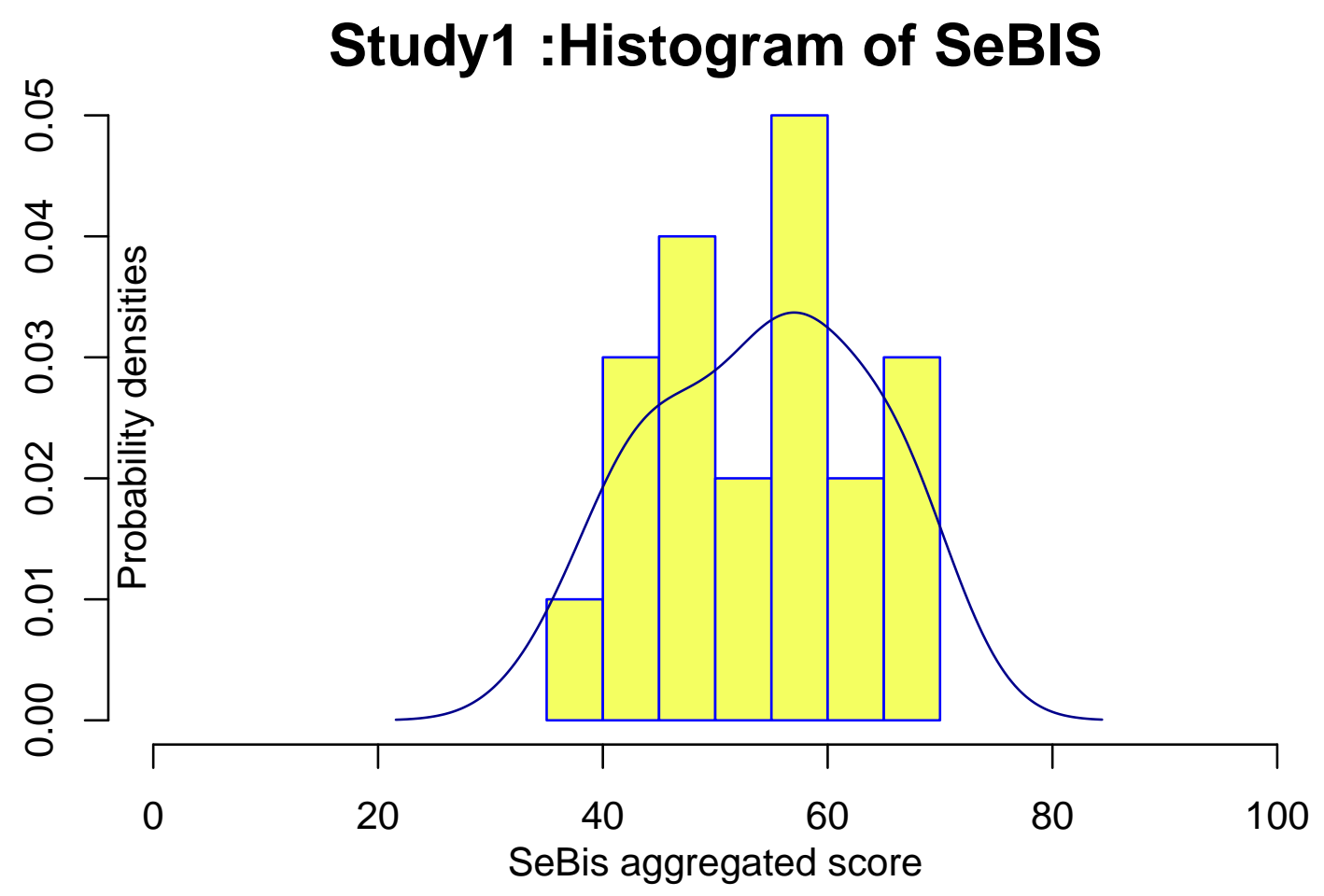

Figure 3.2: SeBIS questions

\section{What is a data breach? Who is responsible?}

In response to being asked what is a data breach, all participants had a general understanding of when an unauthorized party gets access to the secure or private/confidential information. For example, P11 defined data breach as: "Internet data breach is when information is kept privately on the internet and is then accessed by someone who is not supposed to have access to the information.". In response to the question "Have you ever heard of an Internet data breach?", 17 participants said yes, one said no, and two said they are not sure. Among the world's biggest data breaches (see Figure 3.3), participants remembered the Yahoo, Linkedin, Amazon, and facebook breaches. Two participants talked about the Algonquin college loss of students' information, and the other incidents that were mentioned is as following: Edward Snowden [68], Heartbleed [69], and Ashley Madison [63]. When we asked them how they have heard of these data breaches, they mentioned that they follow the news to read about the incident or they have read the breached notifications or have heard from friends.

When asked how would they know if they had been affected in an Internet data 


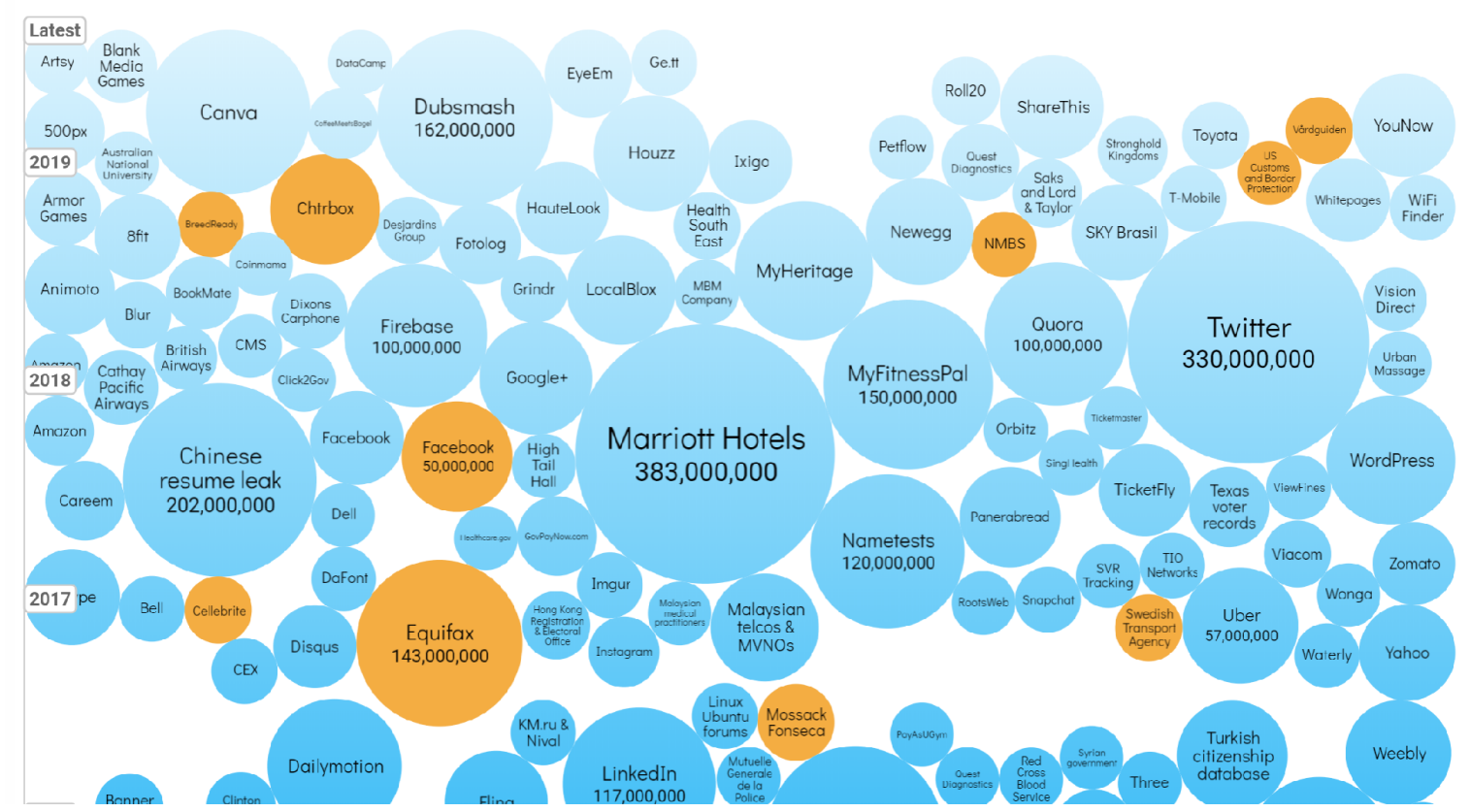

Figure 3.3: World's Biggest Data Breaches \& Hacks from Information is beautiful [23]

breach, eight participants said that they would know if the breached company notified them but they were not sure if there is an obligation to notify the victims. Six participants said that they would know by receiving an alert in their breached account, checking their bank transactions; 6 other participants believed that if the breach happens properly, they would never know that their personal information had been breached. Following the news, being a victim of blackmailing and deletion of their information is also a sign for them that their data had been breached (see Table 3.4). When asked who is responsible for data protection, all participants said users, 18 also considered the companies responsible, four mentioned governments too and one participant believed security admin, expert security groups, and developers are also responsible for protecting data.

\section{Causes of a data breach}

We asked about causes in an open-ended and in a Likert scale format. Participants generally mentioned the following items in their answers: negligence, poor password, poor security measures, and vulnerability. Participants used the word "vulnerability" in a different context as is common in computer security, they meant the vulnerability 
Table 3.4: How would you know if you have been affected in an Internet data breach?

\begin{tabular}{l|l|l}
\hline Code & $\begin{array}{l}\text { Number of } \\
\text { participants }\end{array}$ & Sample of data extracted \\
\hline \hline Company's notifications & 8 & $\begin{array}{l}\text { "I would hopefully be contacted } \\
\text { by the company who lost my in- } \\
\text { formation and be told what was } \\
\text { taken." -P2 }\end{array}$ \\
\hline Alerts to my accounts & 6 & $\begin{array}{l}\text { "when you are notified about your } \\
\text { activities (transactions, logging } \\
\text { in, etc) on internet through SMS } \\
\text { or email notification." -P19 }\end{array}$ \\
\hline Checking their bank transactions & 6 & $\begin{array}{l}\text { "This can be seen in many ways, } \\
\text { such as a sudden change in your } \\
\text { bank statement that looks bizarre } \\
\text { and unusual purchases have taken } \\
\text { place." -P15 }\end{array}$ \\
\hline Never know & 6 & $\begin{array}{l}\text { "You probably wouldn't know if } \\
\text { the attackers did a good job." - } \\
\text { P5. }\end{array}$ \\
\hline News & 1 & $\begin{array}{l}\text { "I would know I was affected in } \\
\text { an internet data breach because I } \\
\text { might hear about it through the } \\
\text { media." -P2 }\end{array}$ \\
\hline "Mlackmailing victim & 5 & $\begin{array}{l}\text { "My information on the phone } \\
\text { gets removed." -P8 } \\
\text { "Maybe noticing data being used } \\
\text { in a way you don't remember do- } \\
\text { ing? Or they'll tell you, if they're } \\
\text { hoping the breach will make you } \\
\text { do something for them." -P5 }\end{array}$ \\
\hline
\end{tabular}


Table 3.5: Participants extracted comments for causes of a data breach

\begin{tabular}{l|l|l}
\hline Causes & $\begin{array}{l}\text { Number of } \\
\text { participants }\end{array}$ & Sample of data extracted \\
\hline \hline Negligence & 4 & $\begin{array}{l}\text { "Carelessness, on the part of the de- } \\
\text { signer, on the user's part for not be- } \\
\text { having safely." -P1 }\end{array}$ \\
\hline Poor password & 3 & $\begin{array}{l}\text { "Easy to guess passwords, whereby } \\
\text { someone's name or last name is used } \\
\text { as passwords." -P9 }\end{array}$ \\
\hline Poor security measures & 4 & $\begin{array}{l}\text { "A company may cut corners and just } \\
\text { do the bare minimum to get certified." } \\
\text {-P18 }\end{array}$ \\
\hline Vulnerability & 4 & $\begin{array}{l}\text { "Vulnerability, which is having data } \\
\text { that is not encrypted. The hacker tar- } \\
\text { gets vulnerable users more easily" -P3. }\end{array}$ \\
\hline
\end{tabular}

of users rather than systems. 4 participants said the end-user carelessness and lack of knowledge is a cause. They also refereed to insecure design and coding of software as carelessness (see Table 3.5).

The box plot in Figure 3.4 shows a summary of the results of the causes. Participants strongly agreed that cyber-attacks and Phishing emails are the cause $(\mathrm{Mdn}=5 / 5$, all the represented numbers are median in our data). They agreed that human error, technology failure, unpatched software, negligence, and not updating software were also causes of a data breach (4/5). Peer-to-peer file transfer was moderately agreed upon as a cause of a data breach (3.5/5). Participants were unsure if data encryption and lack of data access monitoring are causes of a data breach.

\section{Consequences of a data breach}

When asked what can happen as a consequence of an Internet data breach, the answers were as follows. Fifteen participants said that financial loss is the main consequence and 10 participants considered the identity theft and invasion of privacy as a potential impact of data breaches. The following items were brought up by only one participant: fraud, loss of trust, national threat, political consequences, and cyberwar.

In the Likert type questions, everyone strongly agreed that credit card fraud, identity theft, and financial loss are the consequences of a data breach $(\mathrm{Mdn}=5 / 5)$. 


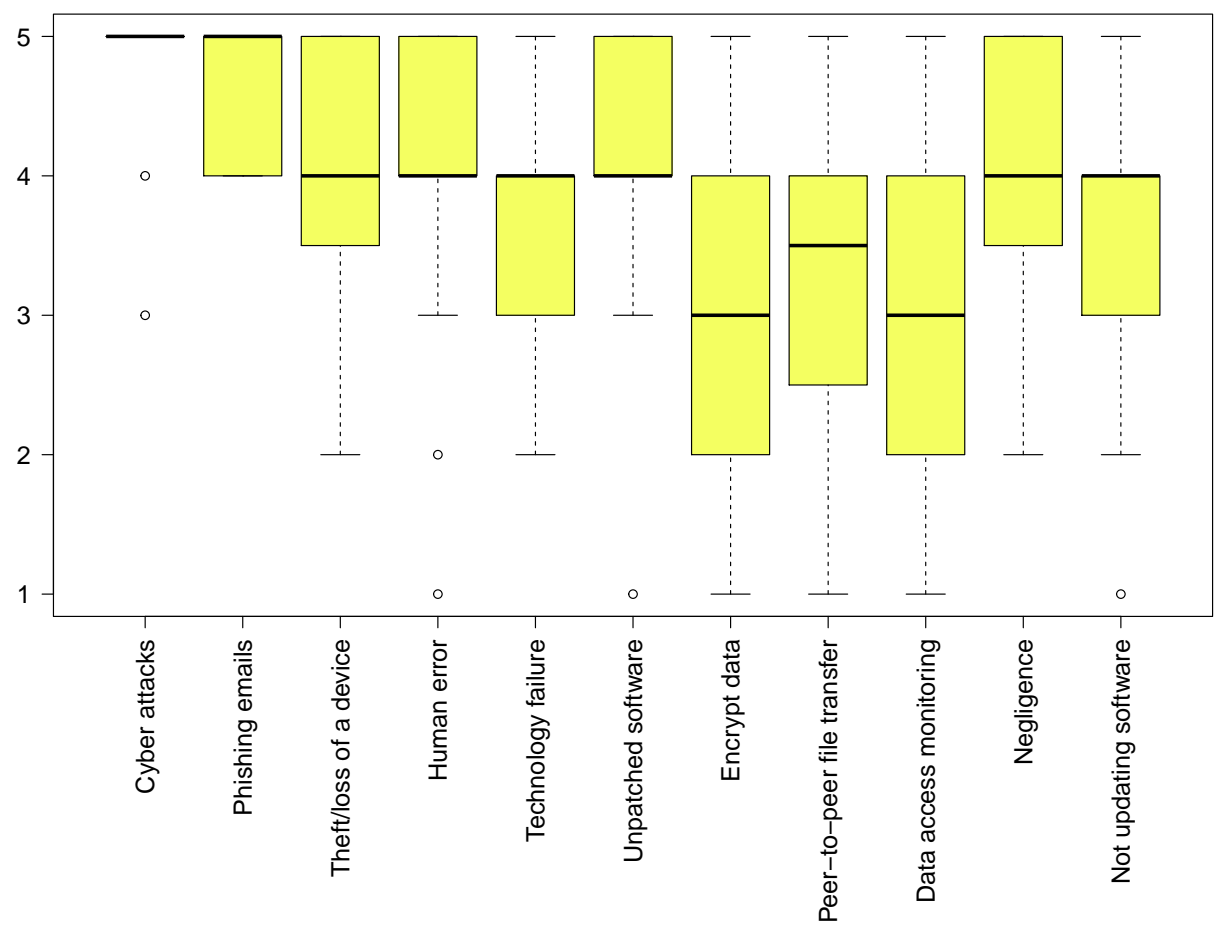

Figure 3.4: What causes an Internet data breach?

They agreed that phishing attack reputational damage, and remote access are also the consequences of a data breach $(4 / 5)$. They were unsure if social engineering is a consequence of a data breach $(3 / 5)$. This indicates that users are aware of those things, such as fraud, can occur because of breach of personal information (see Figure 3.5 .

\section{Motivation of a data breach}

In response to being asked what purpose is behind an Internet data breach, fifteen participants said that it happens because of financial gain, five said to harm the others, three participants also wrote that it also happens because of political purposes and stealing valuable information. Only one or two participants had a more broad perspective of what else can motivate an attacker and mentioned for fun, damaging corporation reputations, spying purposes, proving a weakness in a system, and showing off their technical skills to the companies.

In response to the Likert scale questions, participants strongly agreed that financial gain is the main reason $(\mathrm{Mdn}=5 / 5)$. They agreed that spying purposes, harming 


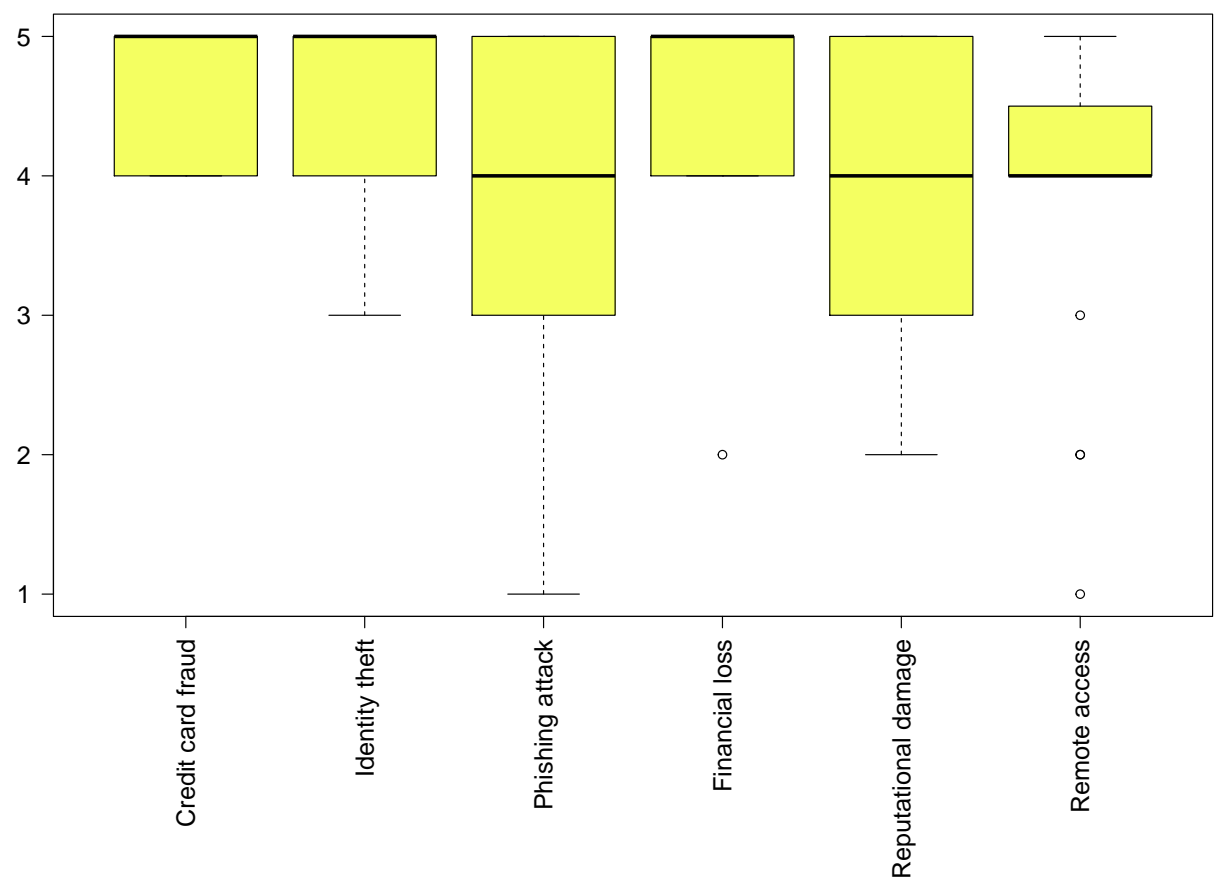

Figure 3.5: What are the consequences of Internet data breach?

others, stealing valuable intellectual property, damaging corporate reputation and fun are other reasons $(4 / 5)$. (see Figure 3.6

\section{Prevention methods}

When asked about what should companies do to protect their data from data breaches, fifteen participants said that companies should invest in security measures, seven said that educating users and employees can also be helpful. Five participants believed that restricting data access can prevent a data breach. Three participants mentioned that companies should monitor regularly their networks. Two participants said that companies have to notify the customers when a data breach happens and encrypt their data using strong encryption algorithm to prevent it. Hiring reliable employees and not collecting all data was considered by one participant as prevention methods of a data breach.

In Likert scale questions, most participants were sure $(\mathrm{Mdn}=4.5 / 5)$ that restricting user authorization, accessibility, regular audit, and assessment can prevent data breaches. Participants were also sure $(4 / 5)$ that it is very likely to prevent a data 


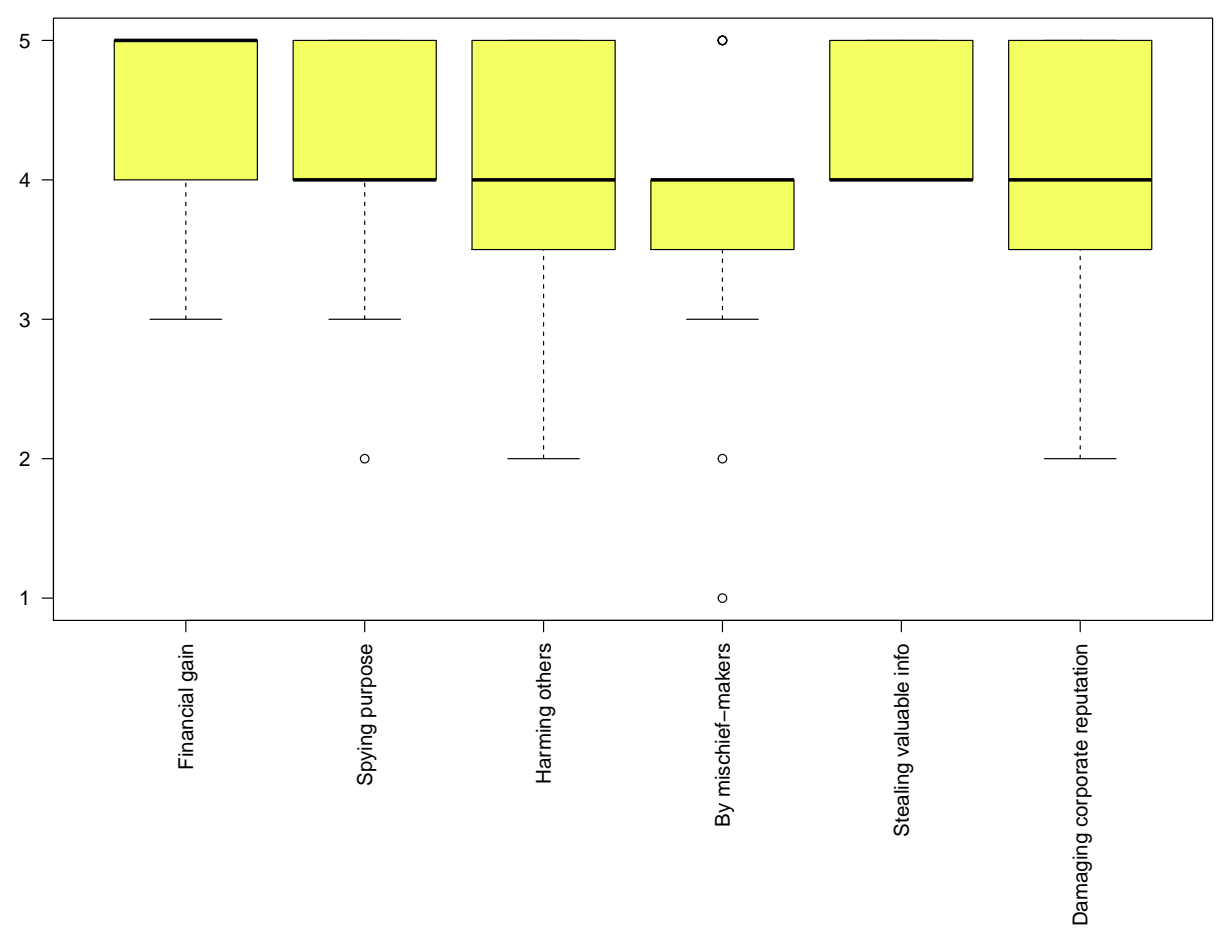

Figure 3.6: Why do data breaches happen?

breach using the following practices: issuing new legislation that keeps up with the threat landscape, installing and updating antiviruses regularly, using data encryption, restriction on data retention, and company policy for equipment use and data security. However, the lower quartile for legislation $(2 / 5)$ is suggesting that participants hold quite different opinions about this aspect (see Figure 3.7). Approximately fifty percent of the participants believed that legislation can not prevent a data breach or they were not sure if it is a preventive method.

In response to being asked what they can do if their personal data has been breached, fifteen participants said that they will change their passwords, seven said that they will contact the police. Three participants said that they will freeze their credit, and only one participant mentioned the existence of "identity theft protection service".

\subsubsection{Drawing Task}

Our goal in this part of the study had been to explore the user's perception of how a data breach happens. To do so, we gave participants the following elements: admin, 


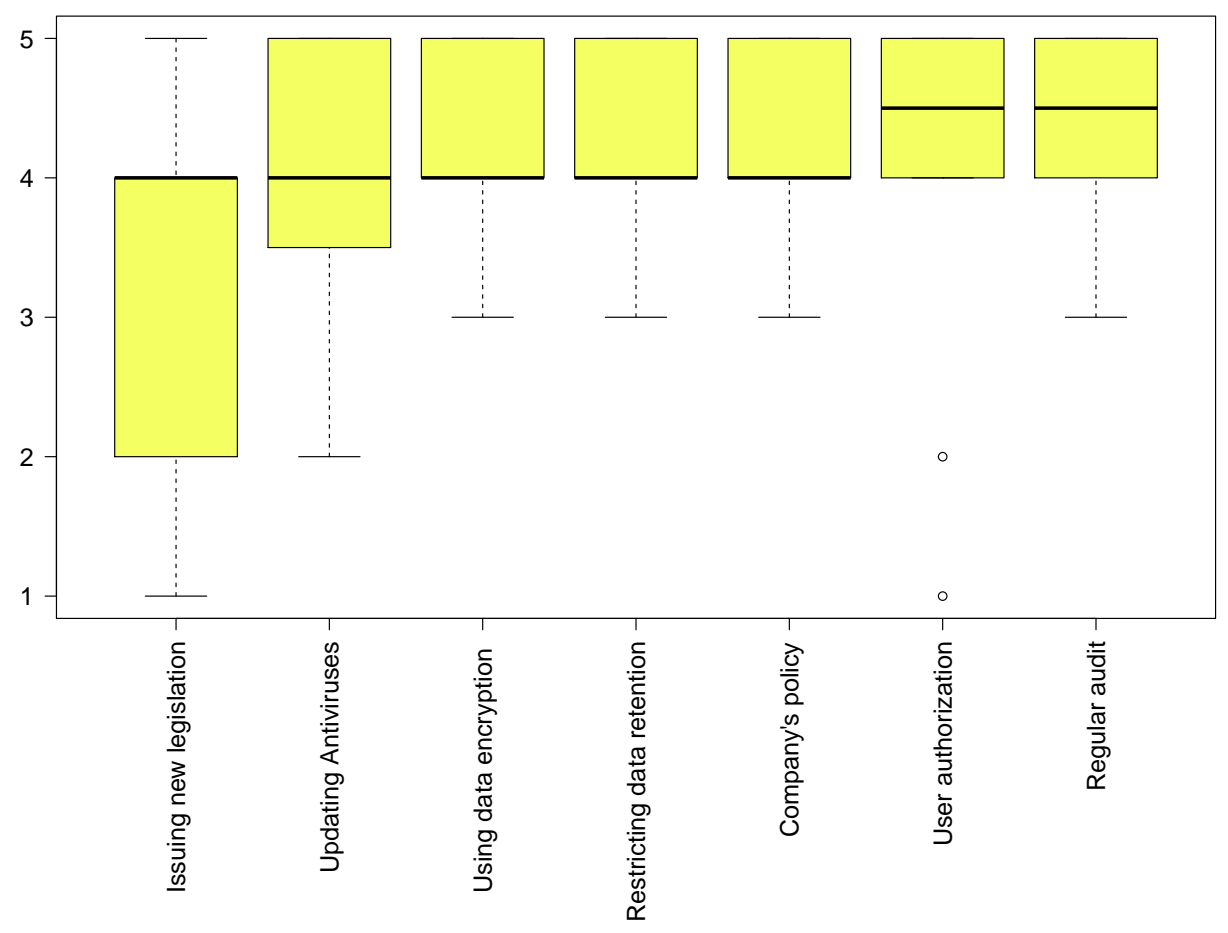

Figure 3.7: How likely is it to prevent a data breach via the following practices?

data, customer, hacker, access to data, vulnerability and asked them to define what are these elements' role in a data breach. We identified three major patterns in the participants' drawings: the first is that an attacker gains admin access, the second is that the attacker gains user access, and the third is backdoor access. The below sections describes these patterns in more detail.

\section{Admin access}

Participants depicted their understanding of how an attacker will gain access to the data as attacking the admin system directly and gain any admin level access to the data. They also believed that accessing the admin system will happen by either a capture attack on the admin password, or guessing the admins' password. Participants were not sure about the level of the admin access and one mentioned that admin maybe have access to only users' identity verification data, so when an attacker attacks the admin account, they will get access to that data and will be able to get access to the server as a normal user (see Figure 3.8. 


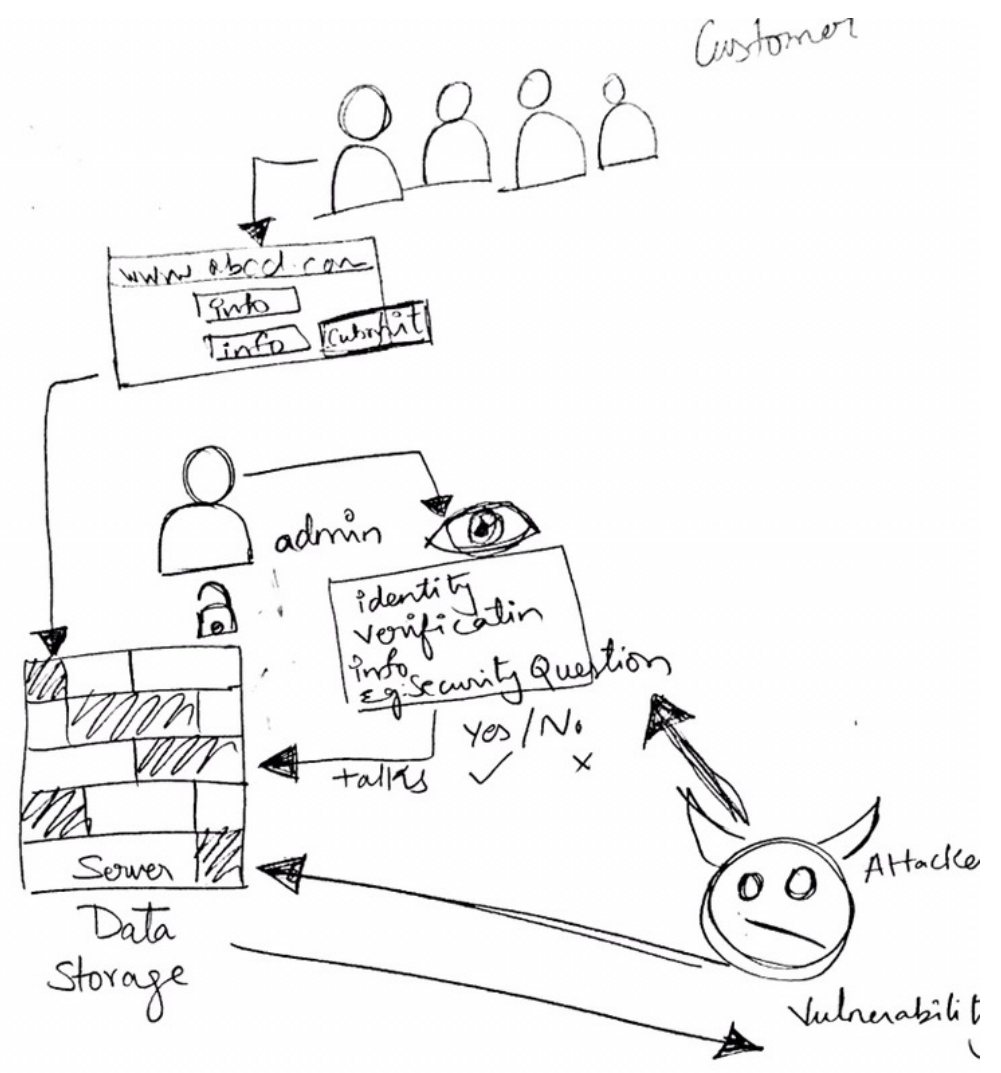

Figure 3.8: P19. Attacker hack the admin system to steal identity verification data. 


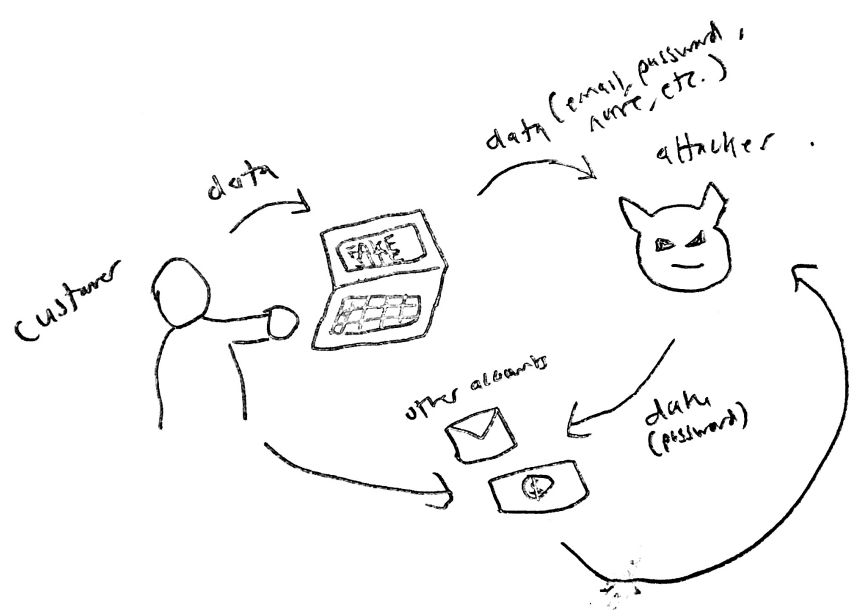

Figure 3.9: P14. Attacker hack the user system to steal personal data.

\section{User access}

This group of participants was focused on when attackers try to hack any individual's data. They also believed that it can happen by a password guessing or a password capturing attack. Attackers may attempt to take screen captures of the users personal computer to gather information over the course of an operation or input capture like keylogging to steal users' credit card information while they are doing online shopping in an apparently legitimate website (see Figure 3.9).

\section{Backdoor access}

In a few cases, participants' drawings of a data breach illustrate an existing backdoor that could be of value for the attackers. In this case, an attacker sees a protected sign in the front-end that give access to the secured data if you have the appropriate "key" (customer and admin). So, the attacker looks for the backdoor and "tries the knob". The backdoor can appear as a result of malware or by an intentional manufacturing decision. However, one participant tried to show that attacker can gain access to the back door by brute force attack, which is in contrast with the concept of backdoors 


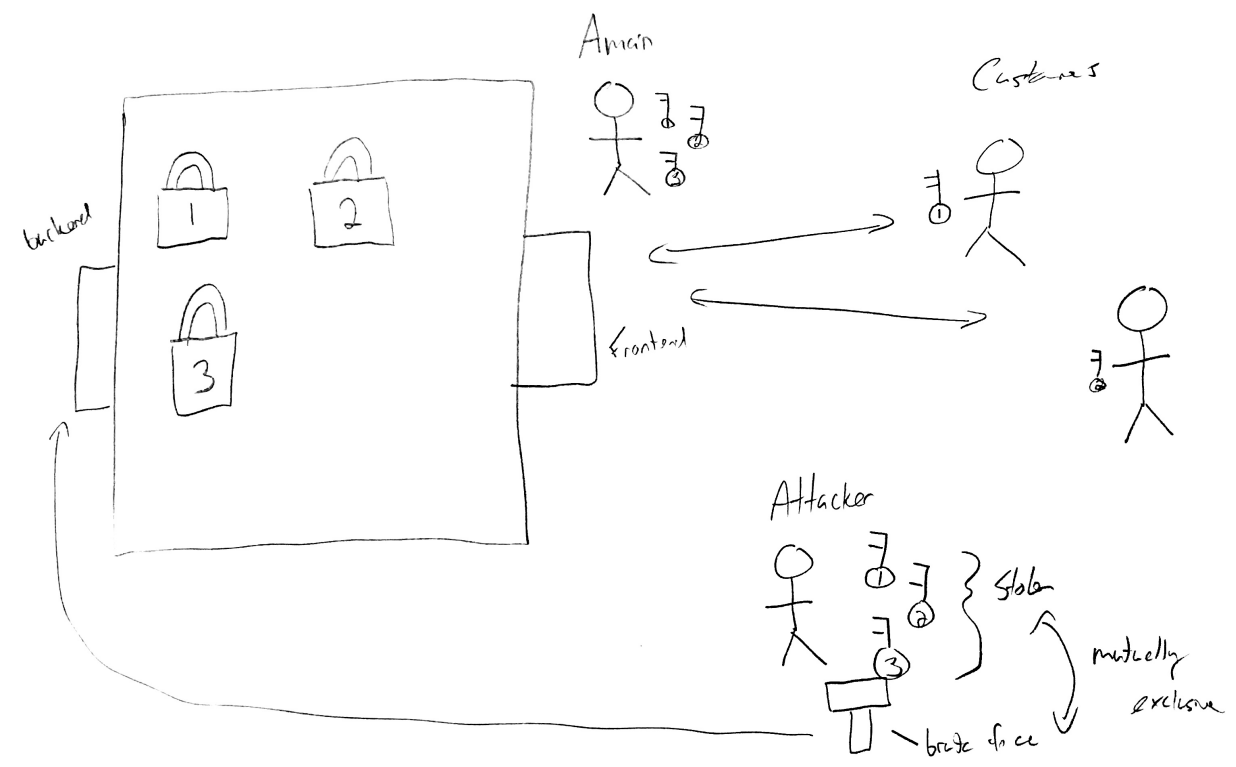

Figure 3.10: P5. Backdoor access.

that are put in place on purpose by manufacturers or cybercriminals to get into and out of a system (see Figure 3.10).

Based on the participants' drawing, it is not really clear if they understand what a vulnerability in a system is or not. Two participants (P2 \& P10) drew the vulnerability as an open door and one as a broken wall (P15) and the others did not include it in their drawings. Only one participant (P13) added more details about the attack's steps where the attacker scans the system for confidential information. The drawing of two participants (P8 \& P17) had some labels about the consequences of a data breach like loss of resources and invasion of privacy.

The main finding of our picture analysis is that most of the participants believe that attackers are interested in their personal data either stored in their devices or shared unintentionally with unauthorized party. For example P8 said: "A data breach happens when a stranger gets access to my private pages such as Instagram and etc. finds my Username and password then uploads pictures and information instead of me"; P20 also said: "A data breach happens When a third party is monitoring your conversations with a server and recording your data without your authorization.". Using elements like customer and data admin did not change their model of how their personal data is disclosed. 


\subsection{Discussion}

The themes we extracted from our participants drawing and other remarks they made have implications for the way a data breach is represented in their mind. We summarize our key insights and discuss the implication of our finding to help the general public to protect their personal information.

\subsubsection{Drawing and open questions analysis}

\section{Attack's target}

We found that the majority of our participants believe that attackers are interested to access their personal information through their social media account, emails, or data that they store in their personal devices like mobile phone or tablet. During the drawing task, most of the people illustrate data in their own possession and ignored the fact that their data can be stored in a company's database where they are using its services or even when they are not direct customers of a company like credit bureaus. Due to this perception, they believed that because users are exposing their data; they are responsible for data protection in the first place and then companies that they are sharing their information with. For example, when asked about data protection responsibility, P8 said: "The most responsible person is ourselves, then it goes to companies we share our information with".

\section{Uncertainty in knowledge}

Participants expressed a great deal of uncertainty about how an attacker gains access to data and their answer were mostly focused on either guessing password or capturing the users' credentials while they are using an illegitimate website, especially for online shopping. Another dimension of uncertainty in people's knowledge is whether or not the protective actions like encryption are strong enough to prevent data breaches. We did not ask directly about the strength of encryption algorithm, however, only two participants talked about encryption as a preventive method. Moreover, although they agreed in Likert scale questions that encryption is a preventive method; they noted that some of the breached companies had used data encryption that was broken by the criminal. So our findings is consistent with what was found by $\mathrm{Wu}$ at al. [87] 
regarding the confusion of participants about encryption strength. The end users need to understand the protective capabilities of encryption and expect companies to encrypt their data using strong encryption algorithms.

Participants' perception of vulnerabilities is not clear in our result. They used vulnerability in the context of users being more vulnerable to attack, because they do not have security knowledge, or a system is vulnerable due to lack of protection: "The hacker targets vulnerable users more easily", "when there is no protection against malware and/ or hackers, then the data would be left vulnerable and could potentially be accessed by the attacker". Moreover, only three participants illustrate vulnerability as a broken wall or open door in their drawing. This suggests there is an educational opportunity for users on the possible cause of external and internal data breaches.

Some participants mention that users need to know more clearly about the type of data which is stored in companies databases and how this data is protected. So, effort can be made to make the information flows more transparent; for example GDPR compliance [20], asking companies to state specifically the purpose of data collection, time period of data storage, who can access data, and notifying users of any automated decision making like profiling.

Our findings demonstrate participants' poor knowledge of what they can do when their data is breached. They did not mention any protection mechanism against the negative impact of data breaches, and it is not clear if they know this mechanism exists. They mentioned changing passwords, deleting accounts, or reporting the incident as a follow-up actions for after-breach: "Call the police or email/ report this incident to the company/ Admin or any regulatory body such as investigators. ", "talk to police, bank, ISP, depending on the extent", "Change my passwords, delete accounts and create new ones with a different email". This suggests there should be clear details in the breaches' notification regarding what actions should be taken by individual after a data breach, and what are the potential risks of not following the after breach instructions. Offering free protective action can also be a way to make sure that there is no barrier in following that.

We found that the wording of our drawing task prompt was not helpful enough in guiding the participants to draw how a massive data breach happens and we needed to be more specific. The reason that participants was focused on their personal devices 
to show a data breach could be attributed to our drawing task prompt.

\subsubsection{Questionnaire analysis}

The results of the questionnaire show that people were expressing good security behavior in securing their devices but the scores are lower in updating, proactive awareness and password generation. They correctly identified the users and employees negligence, poor passwords, poor security measures as causes of a data breach. They could predict that financial loss, identity theft, invasion of privacy are the negative impacts of a data breach. A variety of motivations were mentioned in open-ended questions, like showing off their technical skills, showing weakness in a system, for political purposes and many more. They tended to rate issuing legislation and updating antiviruses as less likely to prevent a data breach. Perhaps this responses is due to their unfamiliarity with new laws [20] that can force the companies to invest more in their security measures and also their belief that attackers are smart enough to find a way to penetrate in a system. This suggests there is an opportunity to give more awareness to users on the fact that a considerable number of massive data breaches could be prevented by simple security practices like having updated certificates and patching software promptly.

\subsection{Conclusion}

Users conceptualize security threats and they come up with mental models based on their individual experience. These models shaped their behavior and guided their security related decisions. In this chapter, we presented our finding drawn from 20 study sessions with participants about their perception of data breach.

Results from the questionnaire and the drawing tasks suggested that users have a basic model of how data breaches happen. They show limited detail in their drawing task and it also illustrates that they are not aware of the potential causes of a breach like unintended flaws in a system that can be prevented easily by patching it as soon as it is discovered. Our analysis also revealed the users' lack of knowledge in actions they can do when their data is breached. The surprising finding was users perception of the responsibility, in the way that since they are the owner of data they are accountable for a data breach. Most of them follow the news to read about 
data breach incidents. Media are influential in rising public concerns over a risk and in selecting which incident receive more attention [89]. Although it is difficult to determine the specific effects of the content of media on public perception of risk [89], their considerable role educating users and improving users mental models of data breaches and data protection can not be disregarded. 


\section{Chapter 4}

\section{Study 2: Crisis Communication Analysis}

In this chapter, we apply the image repair and narrative-semiotic methods of research to the analysis of Equifax and Marriott Hotels crisis communications to see how these incidents are reported in the company press releases and the news. We collected data from 88 stories (58 for the Equifax data breach and 30 for the Marriott Hotels data breach) related to these two security breach crises. We defined the following research question to guide our study:

- RQ. Are there differences between press releases, major newspapers and technical news when reporting data breaches?

\subsection{Methodology}

We focused on the Equifax [5] and the Marriott Hotels [6] data breaches for several reasons: first of all, both happened recently (i.e. the Equifax's data breach occurred on May 2017 and the Marriott Hotels incident happened in November 2018). Moreover, since both were massive data breaches involving the exposure of sensitive personal information, they are of great importance. The Equifax's data breach is resolved and the Marriott Hotels case is still under investigation.

Several sources were studied to collect necessary data to answer the research questions. The data presented in this chapter for Equifax is from press releases posted on the organization's official site, technical news sites like The Register (which is a British tech and science news service) and the HackRead website (that centers on technology, security, privacy, surveillance, cyber warfare, cybercrime and first-hand hacking news). We also selected reputable newspapers (i.e. New York Times, The Washington Post, The Guardian) and the United States Government Accountabil-

ity Office's report to congressional requesters, the Congress of United States letter to the Federal Trade Commission and the Office of Management and Budget and 


\begin{tabular}{|l||c|c|c|c|c|c|r|}
\hline \hline Timeline & PR & GAO & R & NYT & WP & G & Total \\
\hline September, 2017 & 2 & 0 & 9 & 6 & 1 & 6 & 24 \\
October, 2017 & 2 & 0 & 7 & 0 & 0 & 1 & 10 \\
November, 2017 & 0 & 0 & 2 & 0 & 0 & 0 & 2 \\
January, 2018 & 1 & 0 & 1 & 0 & 0 & 0 & 2 \\
March, 2018 & 0 & 0 & 2 & 0 & 0 & 1 & 3 \\
April, 2018 & 1 & 0 & 2 & 0 & 0 & 0 & 3 \\
May, 2018 & 0 & 0 & 1 & 0 & 1 & 0 & 2 \\
June, 2018 & 1 & 0 & 1 & 1 & 0 & 0 & 3 \\
August, 2018 & 0 & 2 & 0 & 0 & 0 & 0 & 2 \\
January, 2019 & 0 & 0 & 2 & 0 & 1 & 0 & 3 \\
February, 2019 & 0 & 0 & 2 & 0 & 1 & 0 & 3 \\
March, 2019 & 0 & 0 & 0 & 1 & 0 & 0 & 1 \\
\hline Total & 7 & 2 & 29 & 8 & 4 & 8 & $\mathbf{5 8}$ \\
\hline
\end{tabular}

Table 4.1: Equifax: number of stories per publication and timeline (PR= Press release, $\mathrm{GAO}=$ Government Accountability Office, $\mathrm{R}=$ The Register, $\mathrm{NYT}=$ New York Times, $\mathrm{WP}=$ The Washington Post, $\mathrm{G}=$ The Guardian)

\begin{tabular}{|l||c|c|c|c|c|c|c|c|c|c|}
\hline \hline Timeline & PR & LS & R & TP & SC & HR & F & NYT & WP & Total \\
\hline November 2018 & 1 & 1 & 1 & 1 & 2 & 1 & 0 & 1 & 1 & 9 \\
December 2018 & 1 & 1 & 1 & 0 & 0 & 2 & 2 & 1 & 3 & 11 \\
Jan 2019 & 1 & 1 & 1 & 1 & 1 & 0 & 1 & 1 & 3 & 10 \\
\hline Total & 3 & 3 & 3 & 2 & 3 & 3 & 3 & 3 & 7 & $\mathbf{3 0}$ \\
\hline
\end{tabular}

Table 4.2: Marriott Hotels: number of stories per publication and timeline $(\mathrm{PR}=\mathrm{m}$ Press Release, $\mathrm{LS}=$ Lawsuit, $\mathrm{R}=$ The Register, $\mathrm{TP}=$ Threat Post, $\mathrm{SC}=$ SCmagazine, $\mathrm{HR}=$ Hackread, $\mathrm{F}=$ Forbes, NYT $=$ New York Times, WP= The Washington Post)

the Equifax Minority Report describing the details of the cybersecurity attack. The analysis covers the period from September, 2017 until March, 2019 (see Table 4.1).

For the Marriott Hotels analysis, press releases posted on the organization's official site, technical news websites (i.e. The Register, HackRead, Threatpost, SC Media, Infosecurity Magazine), general News websites (i.e. New York Times, The Washington Post, The Guardian, $C B C$ News) and class action lawsuit documents against Marriott Hotels were taken as units of analysis. The analysis covers the period from November 30, 2018 until January, 2019 (see Table 4.2). 


\subsubsection{Coding}

We first analyzed the sources using image repair theory (see section 2.4) which is an approach for understanding companies communication in crisis situations like a data breach. This theory can be used by critics to critically evaluate notifications produced during crises. The image theory is a viable approach to use in understanding messages that were created during a crisis. This theory is helpful to find if the company is believed to be responsible and if the act is believed to be offensive[10]. Responsibility can appear in different aspects, a company can be blamed for their security practices, the acts that is permitted to happen by an attacker and the companies' poorly performed actions. Perception of responsibility and offensiveness of an action is more important than reality, so businesses try to use different strategies to affect the perception of responsibility. The coding sheet for image repair includes the following strategies as nodes: shift the blame (i.e. another person or organization is responsible for the act), defeasibility (i.e. the act happened due to lack of information or ability), accident (i.e. the act in question happened accidentally), bolstering (i.e. describing positive acts the company have done in the past to reduce the offensiveness of the act in question), minimization (i.e. to minimize the negative feeling associated with the act), compensation (i.e. the action taken to address the victim's needs; Equifax said that it would provide several services to all U.S. consumers free of charge for one year), corrective action (i.e. correcting the source of the problem) and mortification (i.e. releasing official apologies to public).

We used NVivo12, which is a qualitative analysis tool, to code our data. We first imported all the documents into the software, different folders for different sources. We then created our first group of nodes for image repair strategies; we created a case node to represent each strategy, and we gathered references by coding sources at the nodes. Then when we open any node, we would be able to see all the evidence in one place (See Figure 4.1). See Appendix B and C for more details about results.

We have then used the narrative-semiotic method of analysis (see section 2.4.1) to understand how the positioning of agents changes when the narrator changes. The storytelling strategies help to make sense of what the narrator has perceived and experienced and reveals conflicts and changes during a crisis [55]. The Narrativesemiotic approach is a method of analysis to find common pattern in stories, any 


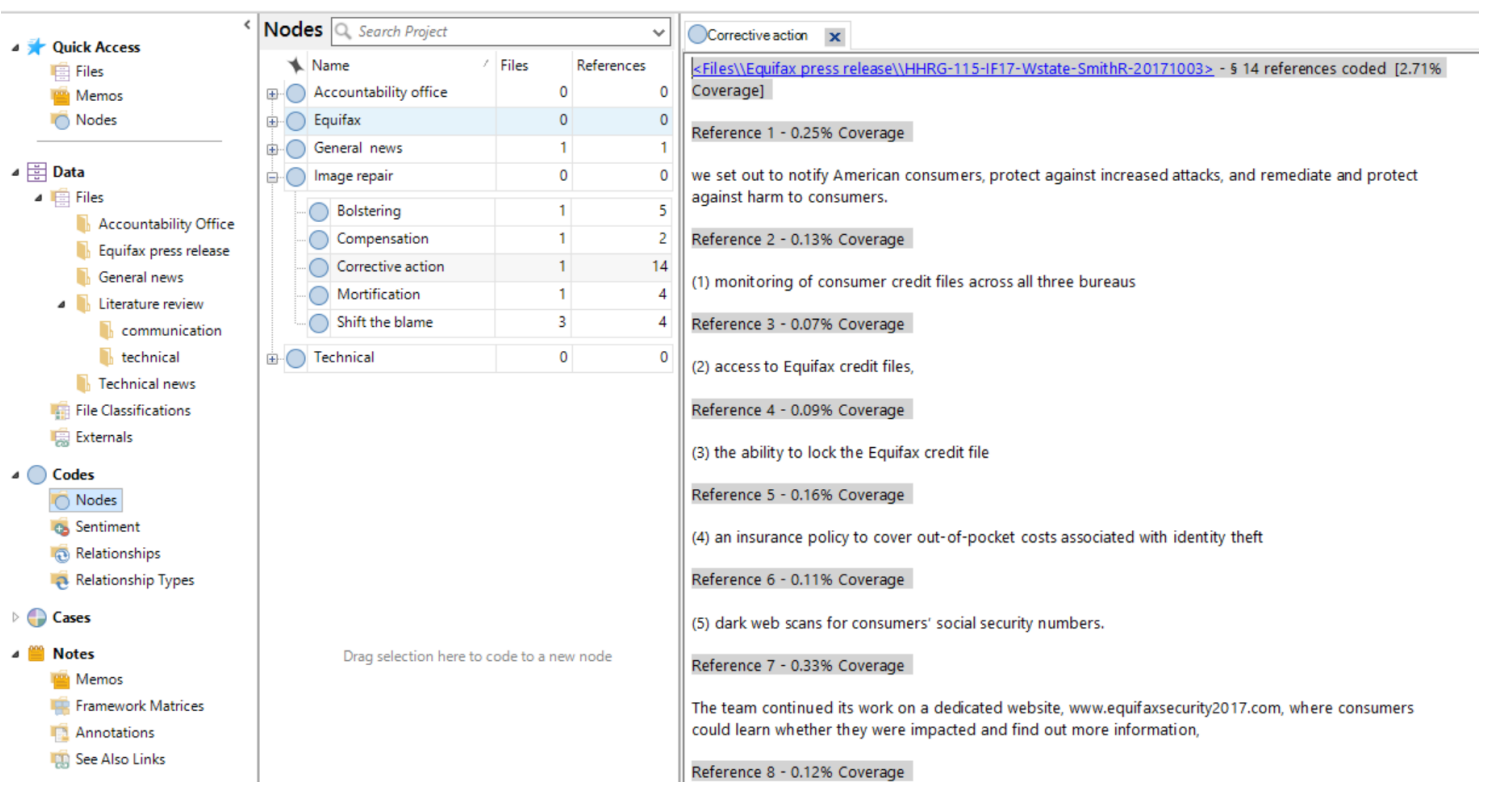

Figure 4.1: Image repair nodes sample

social interaction and reactions to different situations [65]. Based on news framing theory, media and organizations use different features in their messages to frame the crisis [90]. Narrative semiotics is a model, or methodological framework, that can show how these framings are different. This model is valuable for understanding a data breach situation, and the goals and motivations of different narrators of a data breach story. It can help researchers to study a failure of an organization and assess whether the decisions made by the organizations were appropriate or not [67]. We used this method to clarify how companies and news communicate with the general public to help us make sense of what people may have perceived and experienced.

Based on the actantial model (section 2.4.1), the six role categories were applied to analyze the documents that focus on crisis stories from different perspective. To find the patterns in different sources we used the following categories as our nodes in NVivo12: the sender, the subject, the object, the receiver, the helper, the opponent [66] (See Figure 4.2).

\subsubsection{Overview}

To begin, we present a short description of major events and actions in the Equifax and Marriott Hotels stories. 

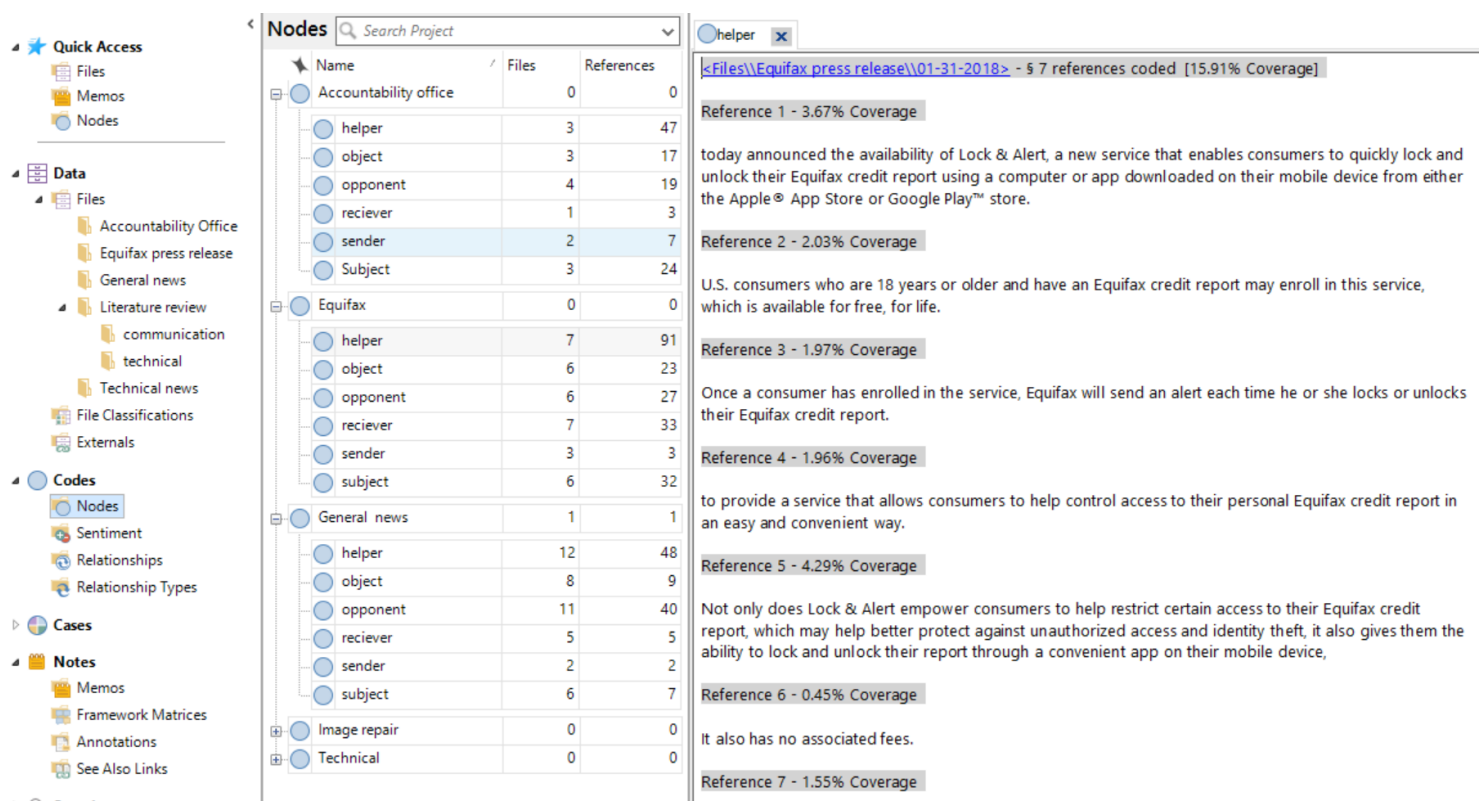

Figure 4.2: Narrative-semiotics nodes sample

\section{Equifax}

The information in this story was taken from press releases posted on the Equifax's official site [5] and the GAO report [77].

The Initial situation Equifax is a consumer credit reporting agency. Equifax collects and aggregates information on over 800 million individual consumers and more than 88 million businesses worldwide, and its database includes employee data contributed from more than 7,100 employers [5].

Complication On 7 September 2017, Equifax announced a cybersecurity incident impacting approximately 143 million U.S. consumers and unknown number in the UK and Canada. Hackers exploited a U.S. website application vulnerability to gain access to certain files in mid-May 2017. They stayed on the system until they were detected on July 2017.

The information accessed primarily includes names, Social Security numbers, birth dates, addresses and, in some instances, driver's license numbers. In addition, credit card numbers for approximately 209,000 U.S. consumers, and certain dispute documents with personal identifying information, for approximately 182,000 U.S. consumers, were accessed. As part of its investigation of this application vulnerability, 
Equifax also identified unauthorized access to limited personal information for certain UK and Canadian residents.

According to Equifax officials, beginning on May 13, 2017, attackers gained access to the online dispute portal (it maintained documents used to resolve consumer disputes) and used a number of techniques to disguise their activity. They extracted a portion of the PII (Personally Identifiable Information) residing on the systems.

After successfully accessing the information, the attackers exfiltrated the data in small increments, using standard encrypted web protocols to disguise the exchanges as normal network traffic. The attack lasted for about 76 days before it was discovered.

Action Taken to Address the Complication Equifax officials stated that, on July 29, 2017, approximately 2.5 months after the attackers began extracting sensitive information, security personnel conducting routine checks of the operating status and configuration of IT systems detected the intrusion on the online dispute portal. A misconfiguration due to an expired digital certificate was the reason why the intrusion was not noticed before. Equifax then blocked several Internet addresses from which the requests were being executed to try to stop the attack. The IT department discovered a vulnerability in the Apache Struts web application framework as the initial attack vector. The US-CERT had notified the company about this vulnerability before this incident. The Apache Foundation also had reported the vulnerability (CVE-2017-5638) 1 in early March 2017.

Equifax took the website offline and then took steps to identify the stolen data and the number of affected people by this incident. Once Equifax officials found out how the attackers were able to access to the company's databases, they took measures to address this problem and avoid it in future. For the challenging task of identifying the affected individuals, Equifax compared the affected database with company's internal databases that were not impacted by the data breach. On March 1, 2018, Equifax stated that approximately 2.4 million U.S. consumers whose names and partial driver's license information were exposed, had been identified.

\footnotetext{
1 "The Jakarta Multipart parser in Apache Struts 2 2.3.x before 2.3.32 and 2.5.x before 2.5.10.1 has incorrect exception handling and error-message generation during file-upload attempts, which allows remote attackers to execute arbitrary commands via a crafted Content-Type, Content-Disposition, or Content-Length HTTP header, as exploited in the wild in March 2017 with a Content-Type header containing a \#cmd= string" 84.
} 
After the investigation, the company notified all U.S. state attorneys general regarding the approximate number of potentially affected residents in each state and its plans for consumer remediation.

On September 7, 2017, Equifax stated in its press release that the company had set up a dedicated website to help individuals determine if their information might have been exposed in the breach. Additionally, Equifax reported that it would provide several services to all U.S. consumers, regardless of whether their information had been compromised, free of charge for one year.

The Resolution The GAO report reveals how Equifax failed to protect Americans' personal data. According to the GAO, "Equifax determined that several major factors had facilitated the attackers' ability to successfully gain access to its network and extract information from databases containing PII," and that "key factors that led to the breach were in the areas of identification, detection, segmentation, and data governance."

Finally, the GAO's report highlights the critical need for legislation to protect consumers whose data is not adequately safeguarded, such as Senator Warren's and Senator Mark Warner's bill to hold credit reporting agencies like Equifax liable for data breaches. Under this legislation, Equifax would have paid at least $\$ 1.5$ billion in penalties for the data breach.

\section{Marriott Hotels}

The information in this story was taken from press releases posted on the Marriott Hotels official site and technical news websites.

The Initial situation Marriott International is an American hospitality company that manages and franchises a broad portfolio of hotels and related lodging facilities [6].

Complication On September 8, 2018, Marriott received an alert from a security tool about an attempt to access their Starwood guest reservation database. Marriott security experts started to determine the situation and they found that the unauthorized access started as far back as 2014. The attackers had copied and encrypted 
information and they took actions towards removing it.

Unauthorized people had access to the Starwood guest reservation database and had stolen information of up to approximately 500 million guests. For 327 million people, Marriott says in its press release that the guests' exposed information includes some combination of name, mailing address, phone number, email address, passport number, Starwood Preferred Guest ("SPG") account information, date of birth, gender, arrival and departure information, reservation date, and communication preferences. For millions of others, their credit card numbers and card expiration dates were potentially compromised. For the remaining guests, the exposed information includes name and sometimes other data such as mailing address, email address, or other information.

Action Taken to Address the Complication On November 19, 2018, Marriott decrypted the information that had been encrypted by the attackers and found that the contents were from the Starwood guest reservation database.

On November $30^{\text {th }}$ 2018, Marriott International announce via Kroll.com (Kroll is a corporate investigations and risk consulting firm) that unauthorized people had access to the Starwood guest reservation database and had stolen information from up to approximately 500 million guests.

Marriott said that it had set up a dedicated website and call center to deal with guests and said it would try to reach affected customers on Friday (November $30^{\text {th }}$, 2018) to inform them of the breach. The site was having problems staying online shortly after the attack was announced.

The company said it was offering one year of free enrollment in a service called Web Watcher to people who live in the United States, Canada and Britain. Marriott described it as a service that keeps an eye on websites where thieves swap and sell personal information and then alerts people if anyone is selling their information.

Marriott said at the end of the announcement that it reported this incident to law enforcement and continues to support their investigation. They had already begun notifying regulatory authorities.

The Resolution The intrusion went unnoticed for four years by Starwood, which was bought by Marriott in 2016 for $\$ 13.6$ billion. There is not much detailed technical 
information about the Marriott breach, and it is still unsolved.

\subsection{Analysis}

In this section, we analyzed the documents of the Equifax and Marriott Hotels data breaches (companies press releases and news), and presented our findings.

\subsubsection{Equifax}

\section{Image Repair}

We evaluated the company's press release during the crisis using the image repair theory. This theory focuses on what are the message options when a company is faced with a crisis [10]. Using these strategies is intended to change the perception of a crisis. See Table 4.3 for the strategies used in press release and illustrations of them. Table 4.3 suggests that Equifax used the repair efforts to minimize the risk of the data breach. Since the company was believed to be responsible for the incident, the CEO blamed the entire thing on IT staff who had not installed an Apache Struts patch issued in the weeks before the hack, and on technology failures. Moreover, the company used other strategies like: bolstering, compensation and corrective actions to reduce the offensiveness of the data breach. As a final general strategy, the CEO apologized to victims.

Table 4.3: Image restoration strategies in Equifax press release [10].

\begin{tabular}{l|l|l}
\hline \multicolumn{3}{c}{ Image Repair Strategies } \\
\hline Strategy & $\begin{array}{l}\text { Key } \\
\text { istic }\end{array}$ & Character- \\
\hline \multicolumn{2}{r}{ Continued on next page } \\
\hline
\end{tabular}


Table 4.3 - continued from previous page

\begin{tabular}{|c|c|c|}
\hline \multicolumn{3}{|c|}{ Image Repair Strategies } \\
\hline Strategy & $\begin{array}{l}\text { Key Character- } \\
\text { istic }\end{array}$ & Illustration \\
\hline Shift the blame & $\begin{array}{l}\text { Another person } \\
\text { did the act }\end{array}$ & $\begin{array}{l}\text { The breach occurred because } \\
\text { of both human error and tech- } \\
\text { nology failures. } \\
\text { The human error was the in- } \\
\text { dividual who is responsible for } \\
\text { communicating in the organi- } \\
\text { zation to apply the patch, did } \\
\text { not do that. }\end{array}$ \\
\hline Bolstering & $\begin{array}{l}\text { Focus on posi- } \\
\text { tive feelings }\end{array}$ & $\begin{array}{l}\text { Equifax was founded } 118 \text { years } \\
\text { ago and now serves as one of } \\
\text { the largest sources of consumer } \\
\text { and commercial information in } \\
\text { the world. } \\
\text { We took data security and pri- } \\
\text { vacy extremely seriously, and } \\
\text { we devoted substantial re- } \\
\text { sources to it. } \\
\text { Equifax is doing everything in } \\
\text { its power to prevent a breach } \\
\text { like this from ever happening } \\
\text { again. }\end{array}$ \\
\hline
\end{tabular}


Table 4.3 - continued from previous page

\begin{tabular}{|c|c|c|}
\hline \multicolumn{3}{|c|}{ Image Repair Strategies } \\
\hline Strategy & $\begin{array}{l}\text { Key Character- } \\
\text { istic }\end{array}$ & Illustration \\
\hline Compensation & $\begin{array}{l}\text { Reimburse } \\
\text { victim }\end{array}$ & $\begin{array}{l}\text { A free credit file monitoring } \\
\text { and identity theft protection } \\
\text { package for all U.S. consumers. } \\
\text { That includes free: 1) credit } \\
\text { file monitoring by all three } \\
\text { credit bureaus; 2) Equifax } \\
\text { credit lock; 3) Equifax credit } \\
\text { reports; 4) identity theft in- } \\
\text { surance; and 5) Social Security } \\
\text { Number "dark web" scanning } \\
\text { for one year. }\end{array}$ \\
\hline Corrective action & $\begin{array}{l}\text { Plan to solve or } \\
\text { prevent problem }\end{array}$ & $\begin{array}{l}\text { We set out to notify Ameri- } \\
\text { can consumers, protect against } \\
\text { increased attacks, and remedi- } \\
\text { ate and protect against harm } \\
\text { to consumers. } \\
\text { In recent weeks, vulnerability } \\
\text { scanning and patch manage- } \\
\text { ment processes and procedures } \\
\text { were enhanced. }\end{array}$ \\
\hline
\end{tabular}

Continued on next page 
Table 4.3 - continued from previous page

\begin{tabular}{l|l|l}
\hline \multicolumn{2}{|c}{ Image Repair Strategies } \\
\hline Strategy & $\begin{array}{l}\text { Key Character- } \\
\text { istic }\end{array}$ & Illustration \\
\hline Mortification & Apologize & $\begin{array}{l}\text { I am here today to apologize } \\
\text { to the American people myself } \\
\text { and on behalf of the Board, } \\
\text { the management team, and the } \\
\text { company's employees. } \\
\text { To each and every person af- } \\
\text { fected by this breach, I am } \\
\text { deeply sorry that this oc- } \\
\text { curred. } \\
\text { I sincerely apologize. } \\
\text { I will close by saying again how } \\
\text { so sorry I am that this data } \\
\text { breach occurred. }\end{array}$ \\
\hline \hline
\end{tabular}

\section{Narrative-Semiotic}

The second level of analysis involves the Narrative-semiotic approach. Here we classified the agents in specific roles as they interact in the sequences of actions. Since a series of events can be told differently, the identification of the agents would change according to the role of the narrator.

The initial document that was considered for the narrative analysis was "Prepared testimony of Richard F. Smith before the U.S. House Committee on Energy and Commerce Subcommittee on Digital Commerce and Consumer Protection". We divided Equifax's story into a series of narrative episodes which refer to different stages of the narrative trajectory. The story starts with the talk of the CEO of Equifax as a narrator about the initial situation of the company and how Equifax offered several services to its customers. Consider the following extract from the aforementioned text: 


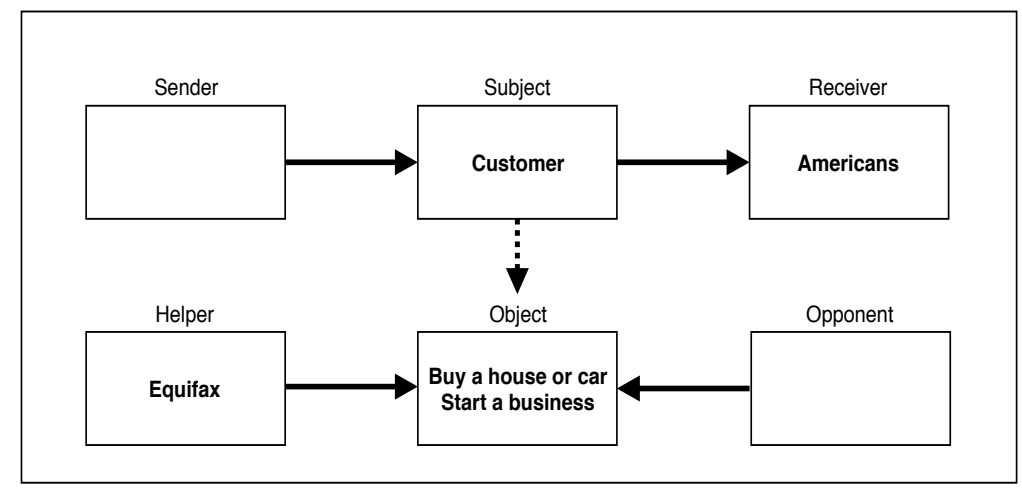

Figure 4.3: Actantial model of Equifax data breach extracted from press releases (narrative program 1)

From Prepared Testimony of Richard F. Smith:

Equifax was founded 118 years ago and now serves as one of the largest sources of consumer and commercial information in the world. That information helps people make business and personal financial decisions in a more timely and accurate way. Behind the scenes, we help millions of Americans access credit, whether to buy a house or a car, pay for college, or start a small business. During my time at Equifax, working together with our employees, customers, and others, we saw the company grow from approximately 4,000 employees to almost 10,000. Some of my proudest accomplishments are the efforts we undertook to build credit models that allowed and continue to allow many unbanked Americans outside the financial mainstream to access credit in ways they previously could not have. Throughout my tenure as CEO of Equifax, we took data security and privacy extremely seriously, and we devoted substantial resources to it.

In this extract, customers (general people or businesses) are described as subjects who want to buy a house or a car, or start a small business. Equifax stepped into the role of helper to give information and to help people make business and personal financial decisions, and millions of Americans are described as receivers. Figure 4.3 shows the actantial model inferred from this text.

In the second stage of Equifax's story, a complication happened due to an external threat (attackers) and Equifax's actions to defend against the intrusion are described in the actions taken to address the complication. Therefore, according to the story, the agent's categories change in the narrative schema. The story starts with the 
repair efforts of the company, as the CEO as a narrator confessed that they failed to protect American consumer data and apologized for the act of data breach. The narrator (Richard F. Smith - Equifax's retired CEO) continued to describe certain actions regarding how this incident happened. From Prepared Testimony of Richard F. SMith: Americans want to know how this happened and I am hopeful my testimony will help in that regard. As I will explain in greater detail below, the investigation continues, but it appears that the breach occurred because of both human error and technology failures. These mistakes - made in the same chain of security systems designed with redundancies - allowed criminals to access over 140 million Americans' data. Upon learning of suspicious activity, I and many others at Equifax worked with outside experts to understand what had occurred and do everything possible to make this right. Ultimately we realized we had been the victim of a massive theft, and we set out to notify American consumers, protect against increased attacks, and remediate and protect against harm to consumers. We developed a robust package of remedial protections for each and every American consumer - not just those affected by the breach - to protect their credit information. The relief package includes: (1) monitoring of consumer credit files across all three bureaus, (2) access to Equifax credit files, (3) the ability to lock the Equifax credit file, (4) an insurance policy to cover out-of-pocket costs associated with identity theft; and (5) dark web scans for consumers' social security numbers.

In this extract, Equifax is foregrounded as the main agent, occupying four positions. Equifax is described as a sender dictating to its employees and outside experts to make the suspicious activity right. Equifax also stepped into the role of receiver, as well as helper. Opponents in this part are criminals, human errors and technology failure. Figure 4.4 shows the actantial model inferred from this text.

Consider, for example, another part of the story where a narrator describes how the complication happened:

From Prepared Testimony of Richard F. SMith:

We now know that criminals executed a major cyberattack on Equifax, hacked into our data, and were able to access information for over 140 million American consumers. (...) Based on the investigation to date, it appears that the first date 


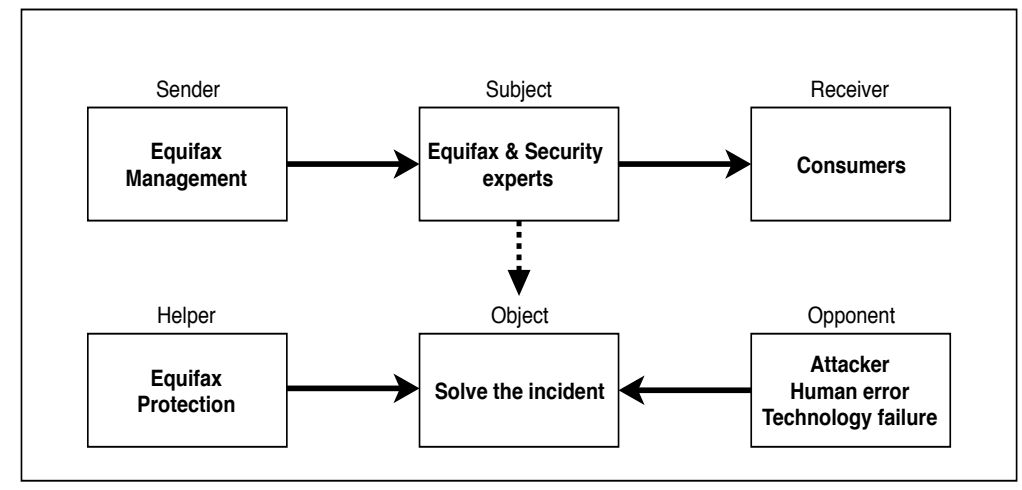

Figure 4.4: Actantial model of Equifax data breach extracted from press releases (narrative program 2)

the attacker(s) accessed sensitive information may have been on May 13, 2017. The company was not aware of that access at the time. Between May 13 and July 30 , there is evidence to suggest that the attacker(s) continued to access sensitive information, exploiting the same Apache Struts vulnerability. During that time, Equifax's security tools did not detect this illegal access.

On July 29, however, Equifax's security department observed suspicious network traffic associated with the consumer dispute website (where consumers could investigate and contest issues with their credit reports). In response, the security department investigated and immediately blocked the suspicious traffic that was identified. The department continued to monitor network traffic and observed additional suspicious activity on July 30, 2017. In response, they took the web application completely offline that day. The criminal hack was over, but the hard work to figure out the nature, scope, and impact of it was just beginning.

The narrator of this press release proposes an attacker as the subject, and accessing to the information is the object. The Apaches Struts vulnerability and the Equifax's security tools that couldn't detect the illegal access are both helpers that assist the attacker. The opponent category in this piece of story includes: the security department investigation, blocking the suspicious traffic, network monitoring, and taking the web application offline. The narrator (the company's CEO) emphasized the company's corrective actions to hinder the attacker in the narrative quest. The following extract focus on the helper role of CERT regarding notifying the companies on the vulnerability that could prevent the whole incident if acted promptly: "On March 8, 


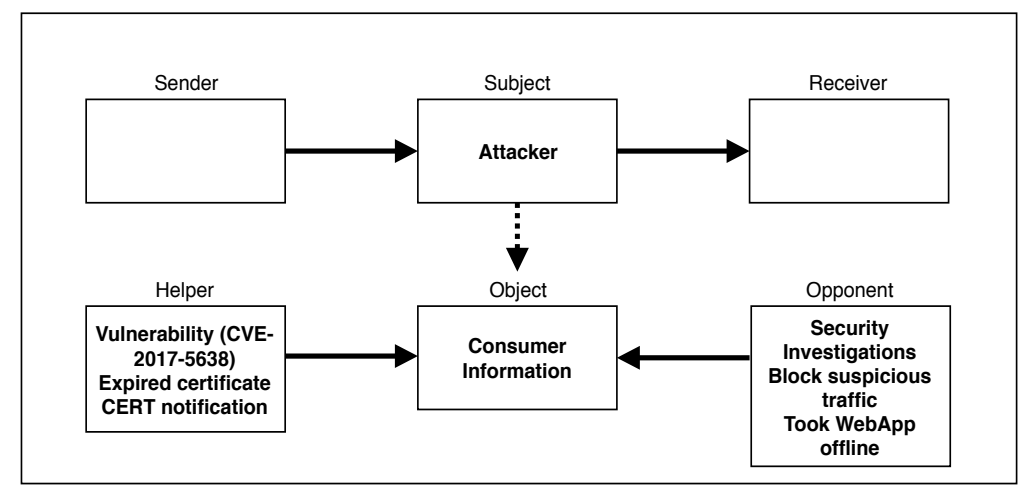

Figure 4.5: Actantial model of Equifax data breach extracted from press releases (narrative program 3)

2017, the U.S. Department of Homeland Security, Computer Emergency Readiness Team ("U.S. CERT") sent Equifax and many others a notice of the need to patch a particular vulnerability in certain versions of software used by other businesses.". Equifax had 5 days to patch the vulnerability before the first date when attackers accessed sensitive information. Since the attacker could exploit the vulnerability of the Apaches Struts, the notification of CERT was a helper to the attacker too (See Figure 4.5).

Figure 4.6 shows the actantial model of the Equifax's story and we can see how the substories are connected to each other. The customer is subject who want to buy something or start a business as object, and Equifax is its helper to achieve the goal. In the other part of the story Equifax stepped into the role of subject who wants to solve the data breach incident, Equifax protection activity is a helper here, and human error, computer failure and attackers are playing the role of opponent. In the last part of the story, attackers are subjects who want to find access to the personal information of Equifax's customers, human error and computer failure is a helper and Equifax protection activity is an opponent.

The secondary documents were the news provided in the technical websites, general news, and the GAO report. After coding these documents using narrative components, we found two narrative programs or mini-narratives that were common among these texts. In the first narrative, Equifax is the subject who wants to solve the incident. The helper category in this mini-narrative that was extracted from the news includes the following components: log files, and new regulations. 

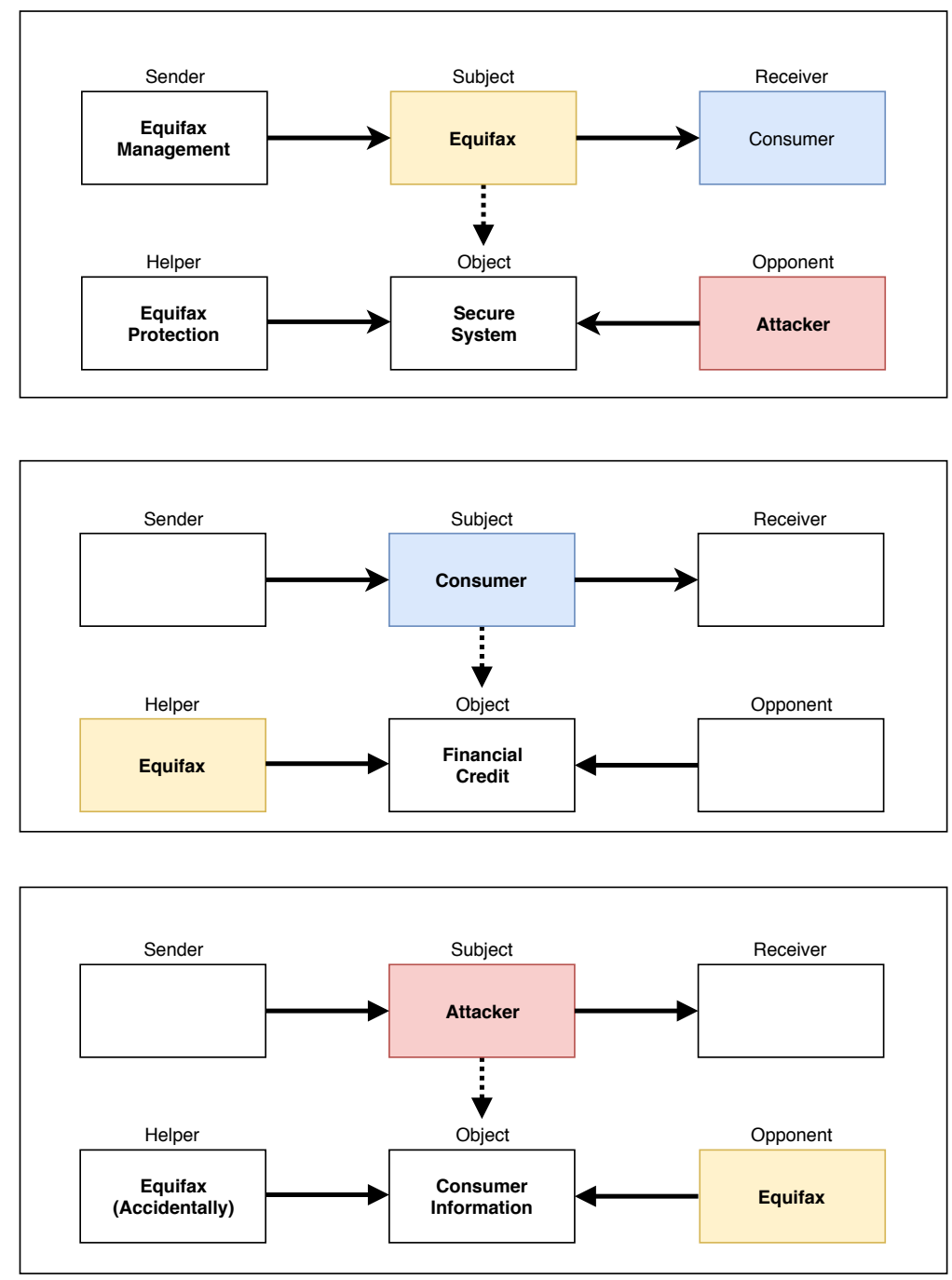

Figure 4.6: Actantial model of Equifax data breach 
Numerous data security failures such as the insecurity of Equifax's web setup, failing to patch the flaw promptly, the lack of restrictions on the frequency of database queries are the first element in the category of opponents. The second one is failure in notifying the data breach victims, the evidence that was extracted from different texts are as following:

FROM GAO EQUIFAX REPORT:

- Equifax executives - including its Chief Security Officer and Chief Executive Officer - kept the public in the dark for more than a month after they found out about the security intrusion.

- The attack lasted for about 76 days before it was discovered.

- Equifax and other big credit reporting agencies keep profiting off a business model that rewards their failure to protect personal information.

- In the three years before the Equifax data breach, the company spent only about $3 \%$ of its operating revenue on cybersecurity-less than the company spent on stock dividends.

FROM THE GUARDIAN:

- because of a process failure in 2016 that meant a limited amount of UK data was stored on the US system between 2011 and 2016.

- Congressman Frank Pallone said Equifax had an "ongoing lax attitude when it comes to protecting consumer data.

- An inadequate response to a data breach that included the personal information of up to 143 million Americans.

FROM ThE REGISTER:

- Equifax was breached in "mid-May" 2017, realized it in July and got around to telling the world in early September.

- Apache Struts was popped, but company had at least TWO MONTHS to fix it. 
- specifically, the lack of restrictions on the frequency of database queries allowed the attackers to execute approximately 9,000 such queries - many more than would be needed for normal operations.

- "As your company continues to issue incomplete, confusing and contradictory statements and hide information from Congress and the public, it is clear that five months after the breach was publicly announced, Equifax has yet to answer this simple question in full: what was the precise extent of the breach?" Senator Elizabeth Warren fumed in a missive late last week.

The third element in the opponent category is inadequate assistance in resolving the problem like no definitive action to hold Equifax accountable, betraying stakeholders by top Equifax executives, an overwhelmed call center, customer response site. Some examples of evidence founded in the documents are as following: FROM THE GUARDIAN:

- It is always a company's responsibility to identify UK victims and take steps to reduce any harm to consumers.

- Ying used confidential information to conclude that his company had suffered a massive data breach, and he dumped his stock before the news went public.

From The ReGister:

That was a hastily constructed WordPress bodge job, and victims were initially asked to agree to take any dispute to arbitration and forfeit the right to take part in any class-action lawsuit.

FROM GAO EQUIFAX REPORT:

- Equifax also failed to provide consumers full protection from new account identity theft.

- These consumer complaints included improper use of credit reports, incorrect information on credit reports, inadequate assistance in resolving problems, and problems with Equifax credit monitoring, fraud alerts, and security freezes in the wake of the breach.

Figure 4.7 illustrates the actantial model of these mini-narratives. 


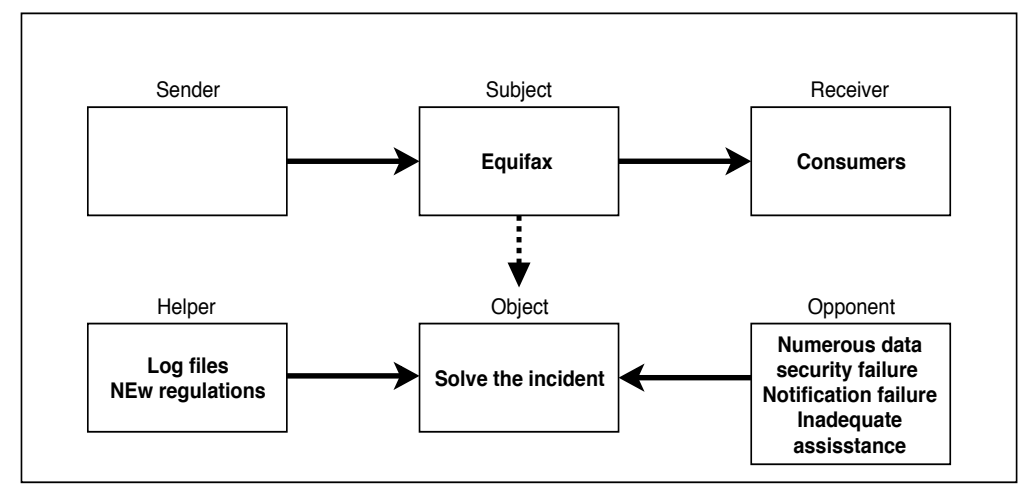

Figure 4.7: Actantial model of Equifax data breach extracted from news and GAO report

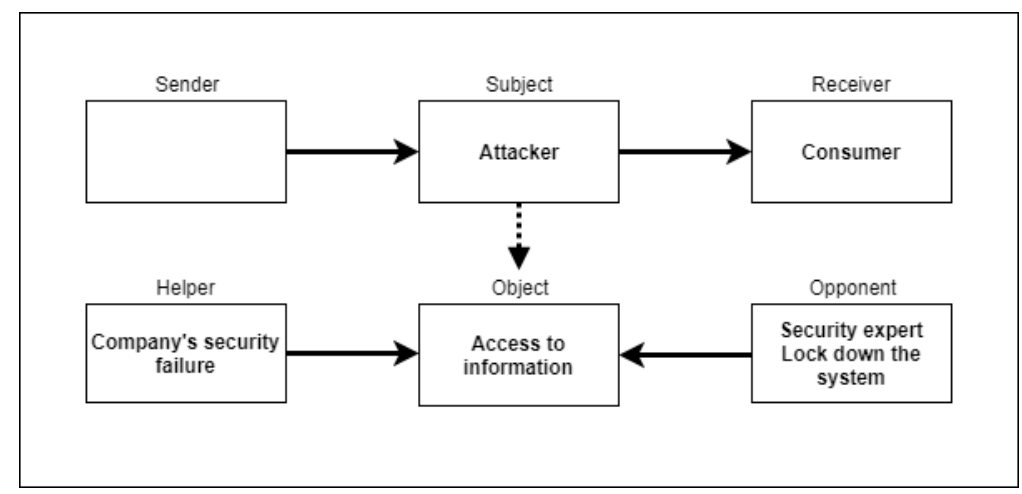

Figure 4.8: Actantial model of Equifax data breach extracted from news and GAO report (narrative program 2)

In the second narrative episode, the attacker is the subject, and getting access to the information is the object. The company's failure to patch the flaw, an expired certificate, doing the malicious activity without being noticed, and lack of restriction on the database are all helpers to the attacker. The opponent category includes locking down the system so that the attackers would not be able to misuse the vulnerability and hiring an expert security team. Figure 4.8 shows the actantial model of this mini-narrative.

Our analysis shows that the narrative programs extracted from company's press release is different from the ones extracted from news. More details will be discussed in a later section. 


\subsubsection{Marriott Hotels}

\section{Image Repair}

For the Marriott Hotels case study, we evaluated the press releases published on the company's website to see what strategies may have been used to change the perception of this incident. Table 4.4 suggests that Marriott Hotels also used the image repair approach to minimize the risk of a data breach and reduce the offensiveness of that. We could not find any evidence for mortification, and we believe that is because the data breach is recent and the company is not believed yet to be responsible for the crisis.

Table 4.4: Image repair strategies in the Marriott Hotels' press release [10].

\begin{tabular}{l|l|l}
\hline \multicolumn{2}{c}{ Image Rpair Strategies } \\
\hline Strategy & $\begin{array}{l}\text { Key Character- } \\
\text { istic }\end{array}$ & Illustration \\
\hline Shift the blame & $\begin{array}{l}\text { Another person } \\
\text { did the act }\end{array}$ & $\begin{array}{l}\text { An unauthorized party had } \\
\text { copied and encrypted informa- } \\
\text { tion, and took steps towards } \\
\text { removing it. }\end{array}$ \\
& Rejecting re- & $\begin{array}{l}\text { Unauthorized access had been } \\
\text { going on since 2014. } \\
\text { Simple denial }\end{array}$ \\
& & $\begin{array}{l}\text { Marriott bought Starwood ho- } \\
\text { tels in 2016. }\end{array}$ \\
\hline
\end{tabular}


Table 4.4 - continued from previous page

\begin{tabular}{|c|c|c|}
\hline \multicolumn{3}{|c|}{ Image Repair Strategies } \\
\hline Strategy & $\begin{array}{l}\text { Key Character- } \\
\text { istic }\end{array}$ & Illustration \\
\hline Bolstering & $\begin{array}{l}\text { Reminds of good } \\
\text { traits }\end{array}$ & $\begin{array}{l}\text { Marriott values our guests and } \\
\text { understands the importance of } \\
\text { protecting personal informa- } \\
\text { tion. } \\
\text { We will continue to work hard } \\
\text { to address our customers' con- } \\
\text { cerns and meet the standard } \\
\text { of excellence our customers de- } \\
\text { serve and expect from Mar- } \\
\text { riott. }\end{array}$ \\
\hline Minimization & $\begin{array}{l}\text { Claim that the } \\
\text { act was not seri- } \\
\text { ous }\end{array}$ & $\begin{array}{l}\text { information for fewer than } 383 \\
\text { million unique guests was in- } \\
\text { volved. } \\
\text { The information was limited } \\
\text { to name and sometimes other } \\
\text { data such as mailing address, } \\
\text { email address, or other infor- } \\
\text { mation. }\end{array}$ \\
\hline Compensation & $\begin{array}{l}\text { Reimburse } \\
\text { victim }\end{array}$ & $\begin{array}{l}\text { Marriott is providing guests } \\
\text { the opportunity to enroll in } \\
\text { WebWatcher free of charge for } \\
\text { one year. }\end{array}$ \\
\hline
\end{tabular}


Table 4.4 - continued from previous page

\begin{tabular}{l|l|l}
\hline \multicolumn{3}{|c}{ Image Repair Strategies } \\
\hline Strategy & $\begin{array}{l}\text { Key Character- } \\
\text { istic }\end{array}$ & Illustration \\
\hline Corrective action & $\begin{array}{l}\text { Plan to solve or } \\
\text { prevent problem }\end{array}$ & $\begin{array}{l}\text { We have taken measures to } \\
\text { investigate and address a data } \\
\text { security incident involving the } \\
\text { Starwood guest reservation } \\
\text { database. } \\
\text { We are supporting the efforts } \\
\text { of law enforcement and work- } \\
\text { ing with leading security ex- } \\
\text { perts to improve. } \\
\text { Marriott is also devoting the } \\
\text { resources necessary to phase } \\
\text { out Starwood systems and ac- } \\
\text { celerate the ongoing security } \\
\text { enhancements to our network. }\end{array}$ \\
\hline \hline
\end{tabular}

\section{Narrative-Semiotics}

Here, we did our second level of analysis which involves the Narrative-semiotic approach. We classified the agents in specific roles as they interact in the sequences of actions based on who is the narrator of the story.

We considered the company's press releases as an initial document for the narrative analysis. The actantial model extracted from the Marriott's press release was focused on the role of Marriott Hotels as sender dictating to the security team to solve the problem and as a subject who wants to solve the data breach crisis. Marriott's quick reaction to solve the incident, protective actions after the incident, like notifying the guests and the regulatory authorities and also decrypting the information to determine the content of stolen data, were considered as helpers. Some sample of the extracted evidence are as follows: 
FROM MARRIOTT PRESS RELEASE:

- Marriott quickly engaged leading security experts to help determine what occurred.

- Marriott was able to decrypt the information and determined that the contents were from the Starwood guest reservation database.

- Marriott reported this incident to law enforcement and continues to support their investigation. We have already begun notifying regulatory authorities.

- Marriott has established a dedicated call center to answer questions you may have about this incident.

- Marriott began sending emails on a rolling basis on November 30, 2018 to affected guests whose email addresses are in the Starwood guest reservation database.

- Marriott is providing guests the opportunity to enroll in WebWatcher free of charge for one year.

The opponent in this document is the unauthorized party who copied and encrypted information and took steps towards removing it (See Figure 4.9). Here is some sample of extracted evidences:

FROM MARRIOTT PRESS RELEASE:

- An unauthorized party had copied and encrypted information, and took steps towards removing it.

- There was unauthorized access to the database, which contained guest information relating to reservations at Starwood properties on or before September 10, 2018.

- An attempt to access the Starwood guest reservation database.

The secondary documents were the technical and general news and class action complaint against Marriott International. We extracted two narratives common 


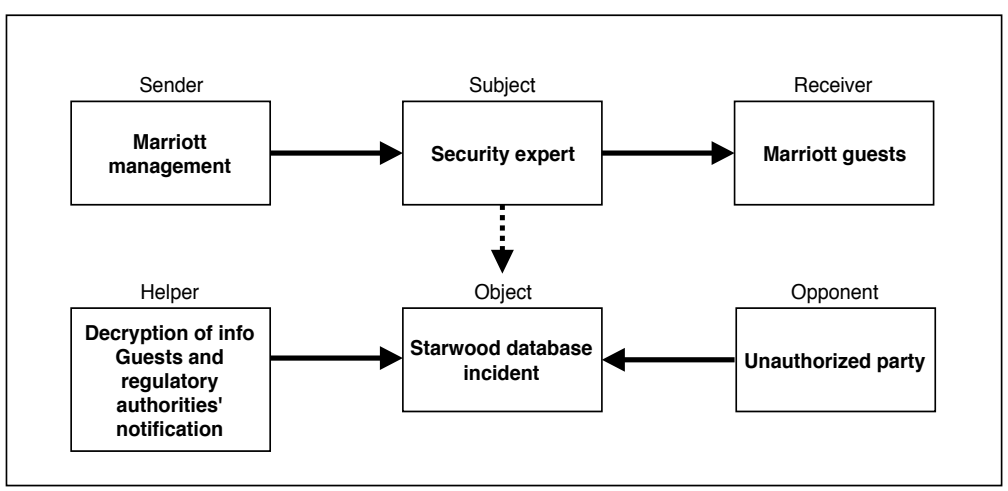

Figure 4.9: Actantial model of Marriott Hotels data breach extracted from company's press release

among these documents. The first narrative can be seen in Figure 4.10, where investigators (a Marriott security team) were the subject, and data protection is the object. The opponent category includes the poor data security practices such as failure to ensure the integrity of the company's servers, failure to safeguard consumer's sensitive information, not discovering the breach for four years, failure in the prompt notification, and significant breach of trust and confidence. Some sample of evidences extracted from different sources are as follows:

FROM NEW YORK TIMES:

- Its Starwood hotel unit did not encrypt the passport numbers for roughly five million guests.

- Marriott said for the first time that 5.25 million passport numbers were kept in the Starwood system in plain, unencrypted data files - meaning they were easily read by anyone inside the reservation system.

From The Washington Post:

Unencrypted passport numbers are valuable to state intelligence agencies because they can be used to compile detailed dossiers on people and their international movements.

From Class ACtion COMPlaint against Marriott International (SOUTHERn District of Florida):

- The data stolen is extremely valuable to identity thieves because it contains elements that can be used to falsely verify fraudulent accounts, like passport 
numbers, specific travel dates, gender, dates of birth, email addresses, phone numbers, and for millions of victims, payment card information.

- While Marriott claim to have encrypted payment card information, they took no similar steps to protect the even more valuable personal information like passport numbers and travel details that they collected.

- Marriott plainly were asleep at the wheel as this data was being exfiltrated from their systems for at least four years while they had no idea the breach was ongoing.

- Marriott negligently left its Starwood computer systems vulnerable to the breach.

- clearly Marriott and Starwood did not and do not take data protection seriously enough to protect their customers information from hackers, or to even know that for four long years, hackers have been stealing their customers sensitive data at will and without detection.

- Instead of promptly detecting and promptly notifying the hundreds of millions of consumers whose personal information was stolen, Marriott said nothing for at least three months between the time the breach was first detected and the time Marriott publicly announced it.

- When Marriott finally did act, it set up a separately hosted website to provide customers information and sent emails from easily spoofed accounts, failing to register for itself domains that cyber-thieves could use to further defraud Marriott customers.

- Although Marriott is offering free credit monitoring to some customers, the credit monitoring services do little to prevent wholesale identity theft.

FROM DARK READING:

- Four years. That's how long an attacker was in Starwood Hotels' databases.

- The delay between when a breach begins and when it's discovered - is an issue across cybersecurity 


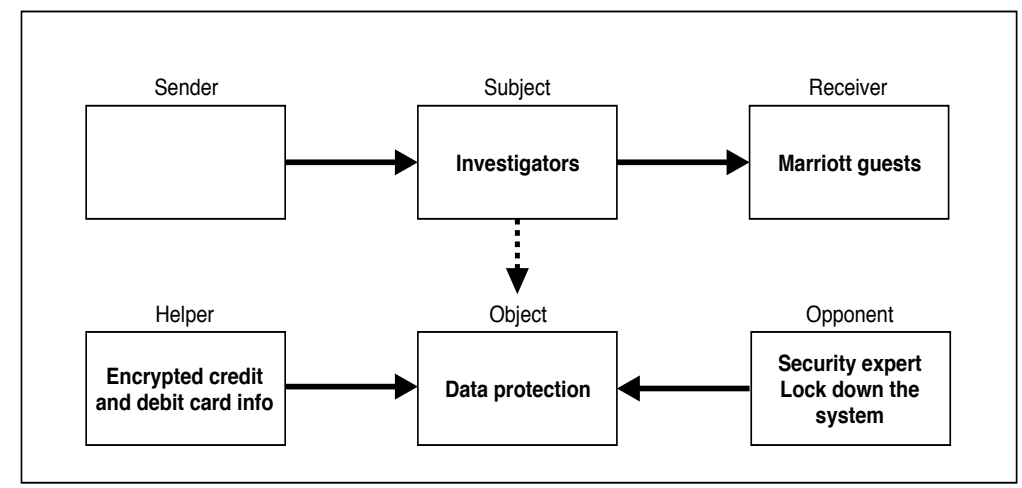

Figure 4.10: Actantial model of Marriott Hotels data breach extracted from news and class action complaint

Encrypted credit and debit card information was regarded as a helper: FROM NEW York TIMES:

The company also said that about 8.6 million credit and debit cards were "involved" in the incident, but those are all encrypted.

In the second narrative program (See Figure 4.11), the attacker is the subject and getting access to Starwood Hotels databases is the object. The company's inadequate cyber due diligence and delay in discovering the attack are helpers. Here are some samples of the evidence:

FROM SCMAGAZINE:

- Malicious actors spent more than four years inside Marriott's Starwood reservation system obtaining access to 500 million guest records that included names, payment card information and other PII, the hotel chain reported today.

- This is yet another example of why it is critical that companies perform cyber due diligence prior to an acquisition or investment. Understanding the cybersecurity posture of an investment is critical to assessing the value of the investment and considering reputational, financial, and legal harm that could befall the company.

Discovering the breach before the time when the cybercriminals attempted to remove data from the U.S. system is an opponent. Here is a sample of the extracted evidence: 


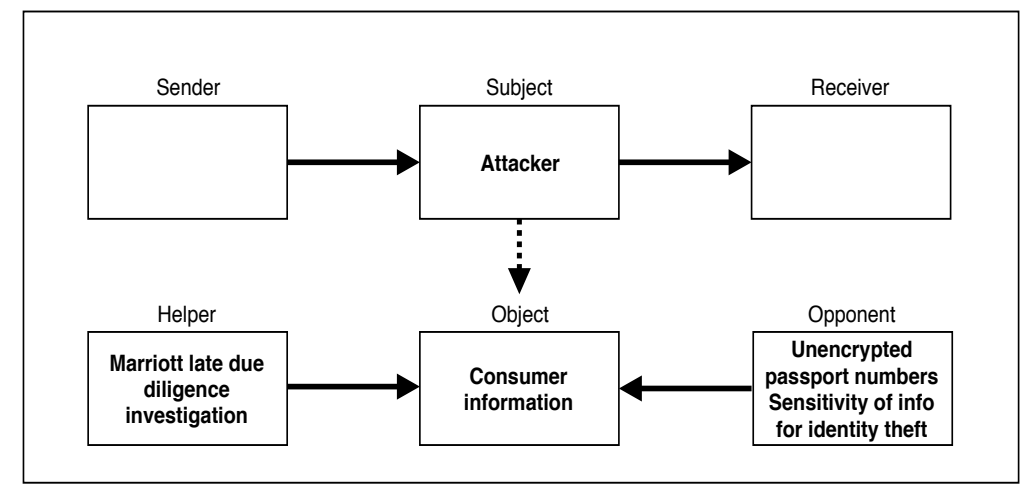

Figure 4.11: Actantial model of Marriott Hotels data breach extracted from news and class action complaint

FROM SCMAGAZINE:

The Marriott IT team only discovered the breach on September 8, 2018 when the cybercriminals attempted to remove data from the U.S. system.

The company has released only one notification regarding its massive breach of data, and it avoids addressing attackers by saying it has no evidence about who the attackers were. That might be because some news and expert started to talk about this breach as part of a Chinese intelligence gathering effort. So, we could not find any evidence in the press release to help us extract the second narrative program. Comparing the first narrative extracted from the news and the one extracted from the press release suggests that they are different. The company's action is the helper in one while it is opponent in the other and news criticized the company's security practices and attitudes towards the victims. More details will be discussed below.

\subsubsection{Discussion}

The primary goal of this study was to examine how the two big companies reported their data breaches. Using image repair strategy, we analyzed press releases posted on the company's official website. We found that Equifax and Marriott Hotels controlled their official announcement appearing on their press releases using communication tactics to reduce the reputational damage and financial losses. The significant difference is that Equifax apologized for the act and was held responsible, whereas Marriott Hotels did not confess that it was the company's fault, but this case is still ongoing. 

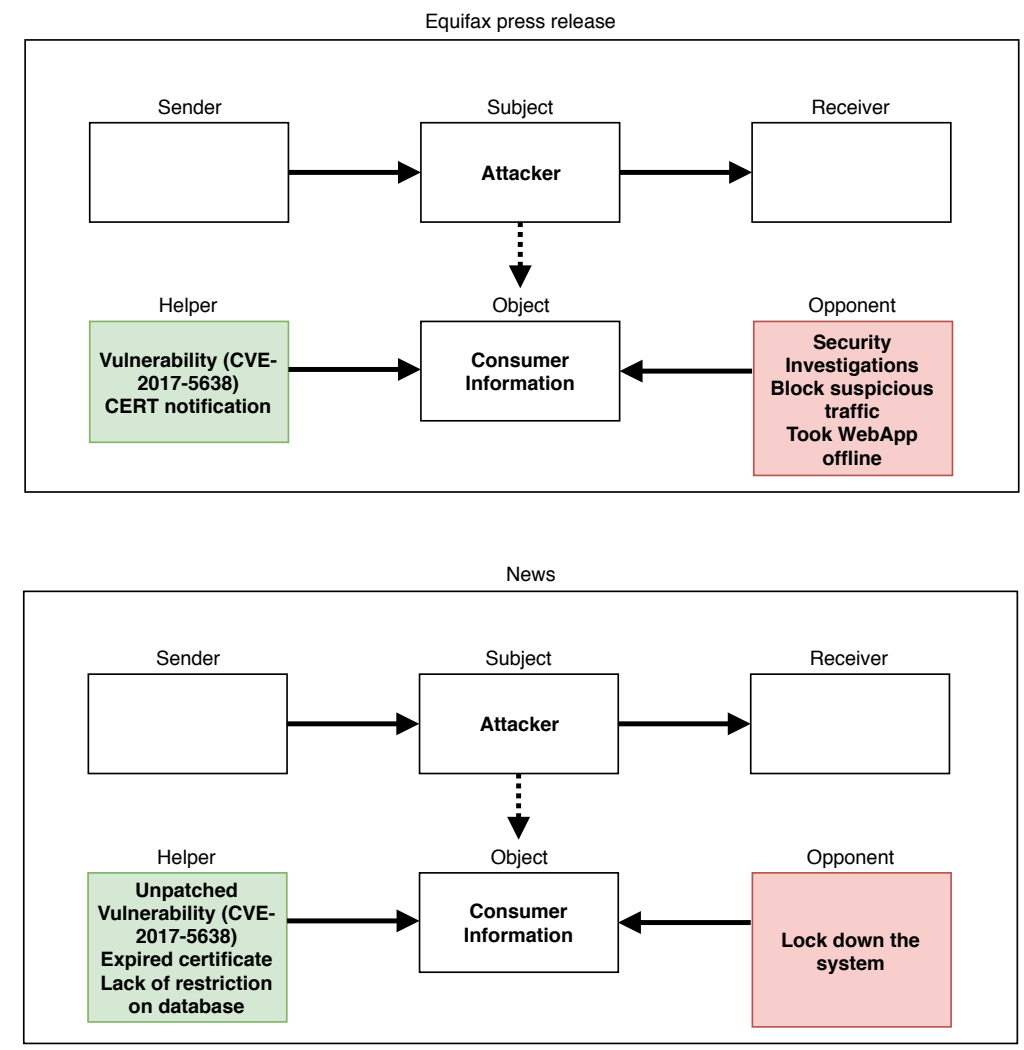

Figure 4.12: Equifax: comparing narrative program 2

Considering the fact that the way media frames a crisis can be different from an organization's press releases, this study detected the dissimilar approaches where press releases and major newspapers were narrating the same story. Most notably, all the agents were not positioned the same way, and we show the conflict in presenting the elements of the narrative.

In the Equifax case, the second narrative program extracted from news is somewhat similar to the Equifax press release, the only difference is that, in the Equifax press release, the expired certificate and lack of restriction on database's queries was not mentioned (see Figure 4.12).

The first narrative story is different from the one that was extracted from company's press releases, in the way that news and GAO report accounted the company's protection actions as an opponent in solving the data breach consequences. Moreover, the media blamed the whole company on the security failure whereas the company blamed the whole sorry mess on a single unnamed IT staff, and the company's lax attitude in crisis response was heavily criticized by the media. The news sees Equifax 

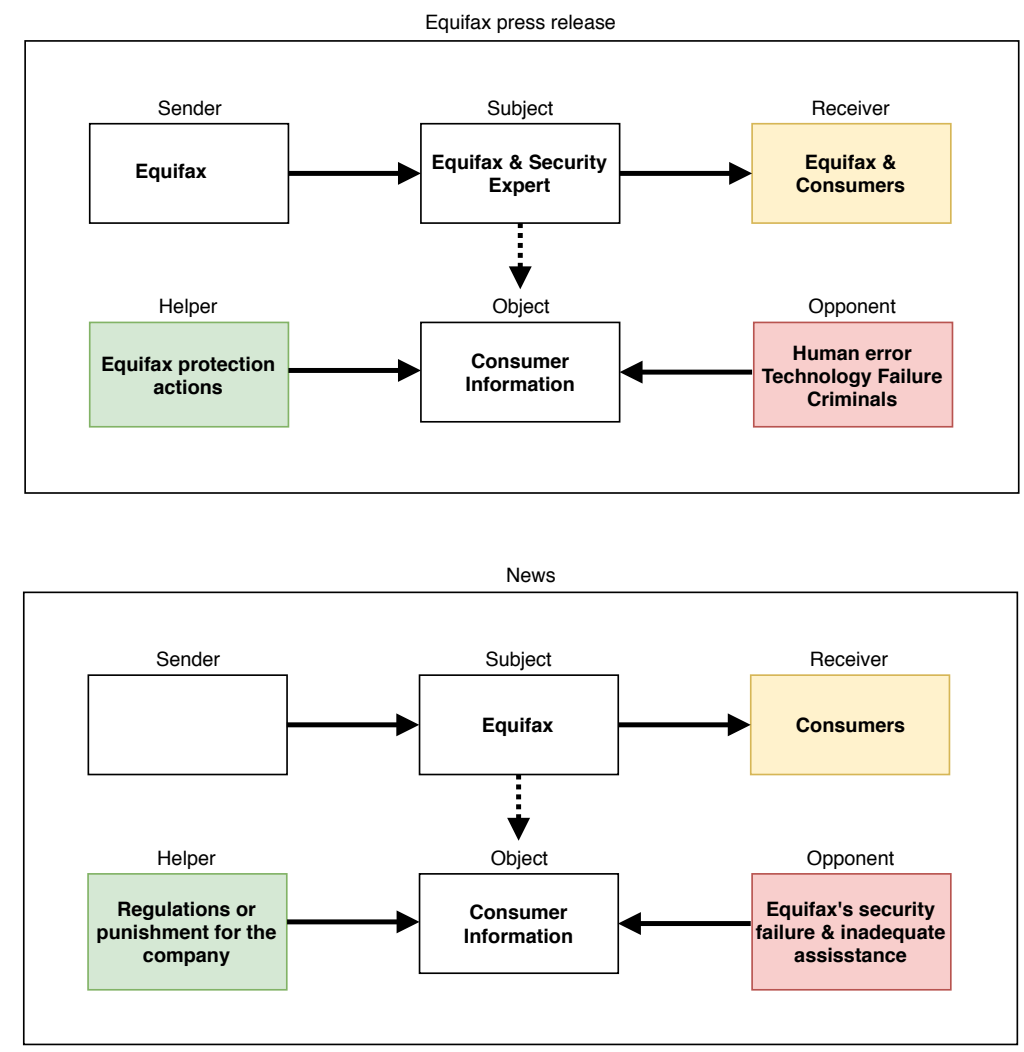

Figure 4.13: Equifax: comparing narrative program 1

responsible for this incident. However, Equifax stepped into the position of a receiver to claim to be a victim (see Figure 4.13) and apparently to reduce its responsibility [21]. .

In the Marriott Hotels case, the company tried to show all of their protective actions as helper to solve the problem, and the four years of attack was totally ignored in the press release, however the news reports were focused on this long dwell time and criticized Marriott for its negligence. The Marriott press release stated that: "Marriott values our guest and understand the importance of protecting personal information (...) We take our obligation to safeguard personal information very seriously." However, the fact that hackers had been stealing their customers sensitive data for four long years, without detection, is in total contrast with the company's statement. It seems to be a strategy to reduce the offensiveness of this incident. The media believed that Marriott unreasonably delayed notifying the victims, whereas the company reported that they notified the authorities and the consumers as soon as possible (see Figure 4.14). Since Marriott did not release any further notification 

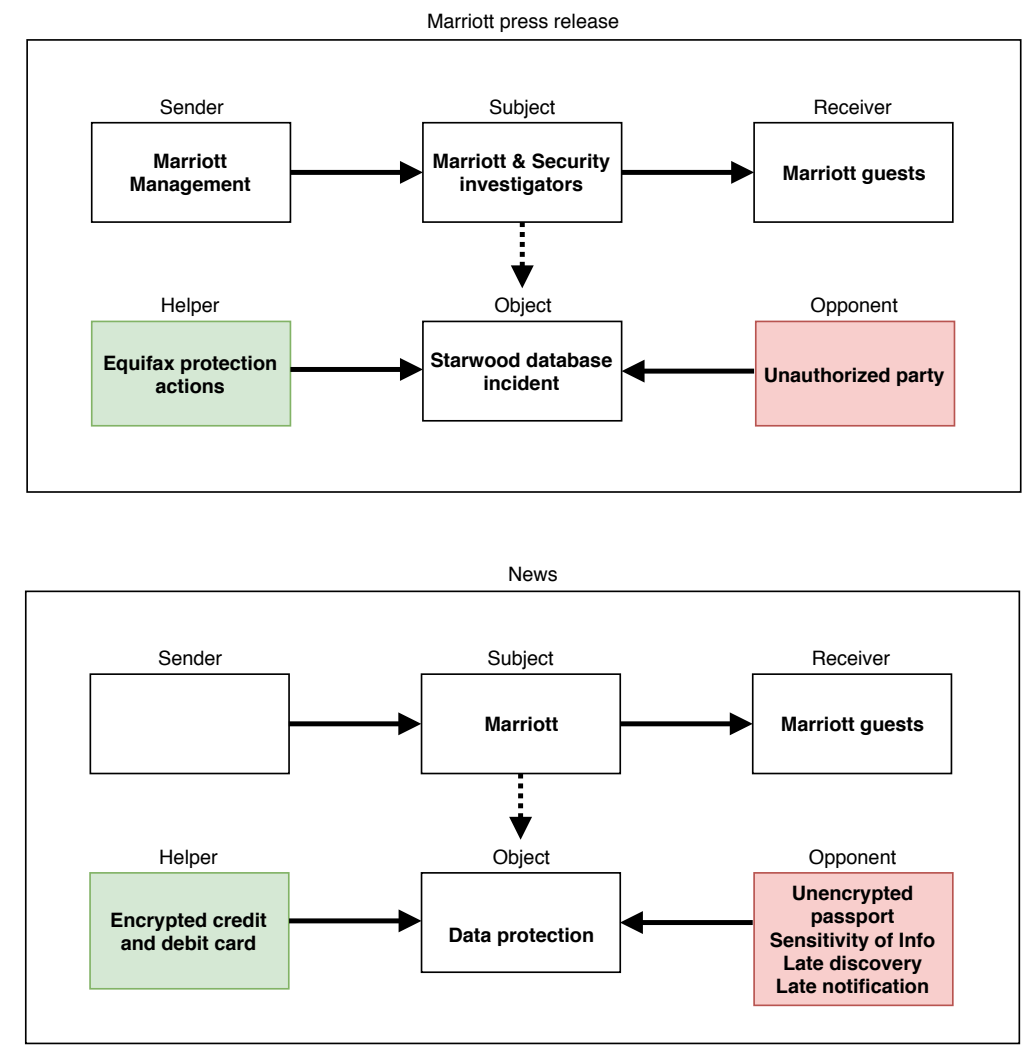

Figure 4.14: Marriott: comparing narrative program

regarding the details of how the attacker accessed the Starwood database, the news media could not focus on the company's attitude in securing data.

All in all, the two companies mapped all their actions to the helper category, while when the narrator changed, this mapping of the actions and agents changed to the opponent category. We show the misfocusing on actants when Equifax was narrating the story; it stepped into the role of the receiver (victim), perhaps to reduce its role responsibilities. In the Marriott case, since the case is open now and there is no legal report about the details of this massive breach, a lot of details are missing.

\subsubsection{Conclusion}

In conclusion, the result of this study suggests that there are dissimilar approaches in terms of the initial response strategies when dealing with a data breach crisis. It also suggests that a breached company tries to deflect the general public by focusing on their good traits and mentioning that the customers and security are of high value for them. However, the news narration of the stories shows that these companies fell 
short in security measures and after-breach actions.

In this chapter, we analyzed the Equifax and the Marriott Hotels data breach's notification to find the strategies that they might have used. We collected data from the companies' press releases posted on their official website, technical news websites and reputable newspapers' site. 58 stories were found for Equifax breach and 30 for the Marriott Hotels' breach. We found that these two companies seemed to use crisis communication strategies to reduce their reputational damage and financial loss. Our analysis also showed that there are differences between press releases, major newspaper and technical news when reporting the same data breach incident. The two companies mapped their after-breach actions into helper category; but the narrator of news reports mapped them into the opponent category. These changes in the actants categories might affects the readers' perception of these data breaches. 


\section{Chapter 5}

\section{Study 3: Quantitative Study on Internet Data Breaches}

\subsection{Introduction}

In this chapter, we explore more about our findings in study 1 and study 2 . In study 1, we explored the users' mental models of Internet data breaches. To this end, we conducted a user study on 20 participants to collect data about their understanding of an Internet data breaches. In study 2, we explored the crisis communication strategies used by Equifax and Marriott Hotels in their data breaches' notification; we also found that there are differences between press releases and news when reporting the same data breach incident. We suspect that this might influence users' understanding of a data breach. The result of these two studies also raised new questions that will be explored in this chapter.

Since there were some unclear results about the users' perception of the responsibility and how they understand a data breach (what are the mechanisms of attack, who are the targets, the regulations, the role of CERT and CVE), we conducted a user study on 100 participants to collect data about the users' understanding of data breaches, the accountability, and the influence of texts from different sources on users. We also asked users to first read data breach descriptions extracted from the Equifax and the Marriott Hotels data breaches and asked their opinion about victims, targets, the companies security measures, and after-breach actions based on the texts .

\subsubsection{Research questions}

Based on our findings in study $1 \& 2$, the following research questions and hypothesis are submitted for study 3 :

- RQ1: Do users know about vulnerabilities, CERT, CVE, and GDPR? Do they find it helpful?

- H1: Based on our findings in study 1, we expect users to show poor model of a 
data breach and might not know about vulnerabilities, CERTs, CVEs. We also think that since GDPR is a European regulation for data protection and our participants are recruited from US and Canada, they might not be aware of it.

- RQ2: How would they rate the accountability, the cause, consequences, motivations, and prevention methods?

- H2: Based on our findings in study 1, we think that users might rate the accountability of the end-user as high as the companies or maybe more.

- RQ3: How might the strategies used in the company's press releases influence the public understanding of data breaches?

- H3: Based on our findings in study 2, after reading the companies descriptions, we expect they perceive the company's actions and security measure helpful and also regarded the company as a victim of the data breach. However, reading the news description can lead to an opposite opinion.

\subsection{Methodology}

We recruited 100 participants through TurkPrime, which is an online crowdsourcing research platform that integrates with Amazon Mechanical Turk (MTurk) 61]. Participants were asked to complete a questionnaire, then read two short extracts and answer the follow-up questions about the incident descriptions. The survey took about 10 minutes on average to complete, and we reimbursed the participants $\$ 2$ for their time. This study was reviewed and cleared by the Carleton University Research Ethics Board (Clearance \#110780). We collected quantitative data through Likert type questions.

\subsubsection{Questionnaire}

We gave participants a group of questions, it would take around 10 minutes to complete. The survey was administered using LimeSurvey (a survey software) on our secure server. Participants read a consent form on the LimeSurvey, and when they accepted it, they proceeded to participate in the following: 
Table 5.1: Security awareness questions. Responses were reported on the following scale: Strongly disagree (1), Disagree (2), Neutral (3), Agree (4), and Strongly agree (5)

1. I have experienced a data security problem, like someone trying to secretly change my password.

2. $\quad$ I have experienced identity theft, like someone applying for credit card using my information.

3. A vulnerability is a weakness which can be exploited by an attacker to perform unauthorized actions within a computer system.

4. A backdoor is a mean to access a computer system or encrypted data that bypasses the system's customary security mechanisms.

5. There are security expert teams around the world that provide timely information about current security issues, vulnerabilities, and exploits.

6. The public announcement of security issues and vulnerabilities can cause a data breach.

7. It is helpful for companies to have a reference list of publicly known information-security vulnerabilities and exposures.

8. It is an opportunity for attackers to have access to a reference list of publicly known information-security vulnerabilities and exposures.

9. In some countries, there are regulations on data protection and privacy for all individuals.

1. Demographic and Security awareness questions: The demographic questionnaire included basic demographic questions same as study 1, and we added the security awareness questions that included some questions about security problems and their security knowledge about Vulnerabilities, CERT, CVE, GDPR (See Table 5.1). We gave the participants some positive statements where we described the aforementioned terms and asked them to indicate the extent to which they agreed or disagreed with those statements.

2. SeBIS: The SeBIS (Security Behavior Intentions Scale) questionnaire included 16-item Scale questions to measure the participants' behaviors towards choosing passwords, device securement, staying up-to-date, and proactive awareness (See Table 3.1), same as study 1.

3. Data breach questions: Participants answered questions about accountability, causes, consequences, motivations, and prevention methods of a data breach which was the same as study 1 . 
Table 5.2: Data breach description

\begin{tabular}{|c|c|c|}
\hline \multicolumn{2}{|c|}{ Instruction } & $\begin{array}{l}\text { Read the following extract and indicate the extent to which you agree } \\
\text { or disagree with the statements. }\end{array}$ \\
\hline \multicolumn{2}{|c|}{ Extract } & $\begin{array}{l}\text { "Throughout my tenure as CEO of the company, we took data security } \\
\text { and privacy extremely seriously, and we devoted substantial resources } \\
\text { to it. The company failed to prevent sensitive information from falling } \\
\text { into the hands of wrongdoers. The people affected by this are not } \\
\text { numbers in a database. They are my friends, my family, members of } \\
\text { my church, the members of my community, my neighbors. This breach } \\
\text { has impacted all of them. It has impacted all of us... We now know } \\
\text { that criminals executed a major cyberattack, hacked into our data, } \\
\text { and were able to access information for over } 140 \text { million American } \\
\text { consumers... Upon learning of suspicious activity, I and many others } \\
\text { at the company worked with outside experts to understand what had } \\
\text { occurred and do everything possible to make this right. Ultimately, } \\
\text { we realized we had been the victim of a massive theft, and we set out } \\
\text { to notify American consumers, protect against increased attacks, and } \\
\text { remediate and protect against harm to consumers. We developed a } \\
\text { robust package of remedial protections for each and every American } \\
\text { consumer - not just those affected by the breach - to protect their } \\
\text { credit information." }\end{array}$ \\
\hline "\# & \multicolumn{2}{|r|}{ Statement } \\
\hline 1. & \multicolumn{2}{|c|}{ The customers of the company are victims of the data breach. } \\
\hline 2. & \multicolumn{2}{|c|}{ The company is the victim of the data breach. } \\
\hline 3. & \multicolumn{2}{|r|}{ The company had a relaxed attitude about protecting customers data. } \\
\hline 4. & \multicolumn{2}{|c|}{ The company takes security measures seriously. } \\
\hline 5. & \multicolumn{2}{|c|}{ Attackers wanted to harm the company. } \\
\hline 6. & \multicolumn{2}{|c|}{ Attackers wanted to harm the customers. } \\
\hline 7. & \multicolumn{2}{|c|}{ The company is helping customers recover from the breach. } \\
\hline 8. & \multicolumn{2}{|r|}{ The company put customers at risk by neglecting data protection. } \\
\hline 9. & \multicolumn{2}{|r|}{ The company is accountable for problems resulting from the data breach. } \\
\hline 10. & \multicolumn{2}{|r|}{ The customers are accountable for problems resulting from the data breach. } \\
\hline
\end{tabular}


4. Data breach descriptions: In this part of the study, we provided three groups of data breach descriptions (2 from the Equifax data breach and 1 from the Marriott Hotels breach), one of these groups was randomly assigned to each participants to read. Each group contained a short text extracted from companies press release and one from news or GAO report. Participants first read the extract, and then they answered 10 Likert scale questions to gauge their opinion about the companies security measures, after breach actions, motivations and responsibility. The order of the texts appearing in each group was also randomized; some participants first read a text from the companies' press release and then a text from the news and some did the reverse order, see Table 5.2 for a sample of the data breach descriptions, and see Appendix for the complete questionnaire.

\subsubsection{Participants}

Participants were recruited using TurkPrime. We recruited 50 participants in the first round to make sure that we are getting reasonable results from the data breach description questions. Then we excluded the first 50 participants from the study using Worker IDs to prevent the same participants to complete the study multiple times [61, and we recruited 50 participants in the second round. We recruited 100 participants in total from Canada (3.84\%) and US (96.15\%); 33 female and 67 male. A summary of their demographics is shown in Table 5.3 .

\subsection{Data Analysis}

During the questionnaire, we asked the participants Likert-scale questions. Each of the questions were scored 1-5, where 1 stands for "Never", "Very low" or "Strongly disagree", 5 for "Always", "Very likely" or "Strongly agree", and 3 for "Sometimes" or "Neutral". We analyzed the results of questionnaire by calculating the median for the central tendency and spread of the distribution of our data. For the data breach description questions, we did a Wilcoxon test to find the differences in participants' responses in each pair of company and news questions. We also used the ANOVA test to see if the source (company and news), order of text within each group, and the group of data breach description affected the participants' responses. 
Table 5.3: Participants' demographics

\begin{tabular}{|c|c|c|c|c|c|c|}
\hline & \multicolumn{2}{|c|}{ Answer } & Percentage & & Answer & Percentage \\
\hline Age & \multicolumn{2}{|c|}{$\begin{array}{c}41-50 \\
51-60 \\
\text { Over } 60\end{array}$} & $\begin{array}{l}36 \% \\
42 \% \\
13 \% \\
3 \% \\
6 \% \\
\end{array}$ & Education & $\begin{array}{l}\text { Less than High school } \\
\text { High school degree or } \\
\text { equivalent } \\
\text { College/ Bachelor's degree } \\
\text { Trade or technical degree } \\
\text { Graduate degree }\end{array}$ & $\begin{array}{l}54 \% \\
5 \% \\
11 \% \\
\end{array}$ \\
\hline & & \multicolumn{4}{|c|}{ Answer } & Percentage \\
\hline \multicolumn{2}{|c|}{$\begin{array}{l}\text { Field of } \\
\text { study }\end{array}$} & & $\begin{array}{l}\text { mal science } \\
\text { ural science } \\
\text { ial science } \\
\text { sineering } \\
\text { s } \\
\text { dicine } \\
\text { ter }\end{array}$ & $\begin{array}{l}\text { computer sc } \\
\text { (Biology, Pl }\end{array}$ & $\begin{array}{l}\text { ience, Logic, Mathematics) } \\
\text { ysics, Chemistry...) }\end{array}$ & $\begin{array}{c}15 \% \\
10 \% \\
16 \% \\
9 \% \\
10 \% \\
3 \% \\
0 \% \\
37 \%\end{array}$ \\
\hline
\end{tabular}

\subsection{Results}

\subsubsection{SeBIS Questions Results}

The boxplots in Figure 5.1 show a summary of the results of SeBIS questionnaire. Participants reported that they lock their devices and use password/PIN to unlock them. They agreed $(M=4 / 5)$ that they use different password for different accounts, they use strong passwords and include special characters. They also reported a high proactive awareness on verifying the URL and checking security problems. They agreed about keeping software and anti-virus up-to-date, but they report a neutral behavior regarding installing updates right away.

\subsubsection{Security Awareness}

The boxplots in Figure 5.2 show a summary of the results of Security awareness questions. We asked participants if they have experienced any security problems, like someone trying to secretly change their password, the median of our data is $4 / 5$, but its boxplot is low-skewed (non-normal distribution). They reported that they had not experienced identity theft $(\mathrm{M}=2 / 5)$. They agreed with the statements representing vulnerability, backdoor (5/5), CERT, CVE, and GDPR(4/5). They also 


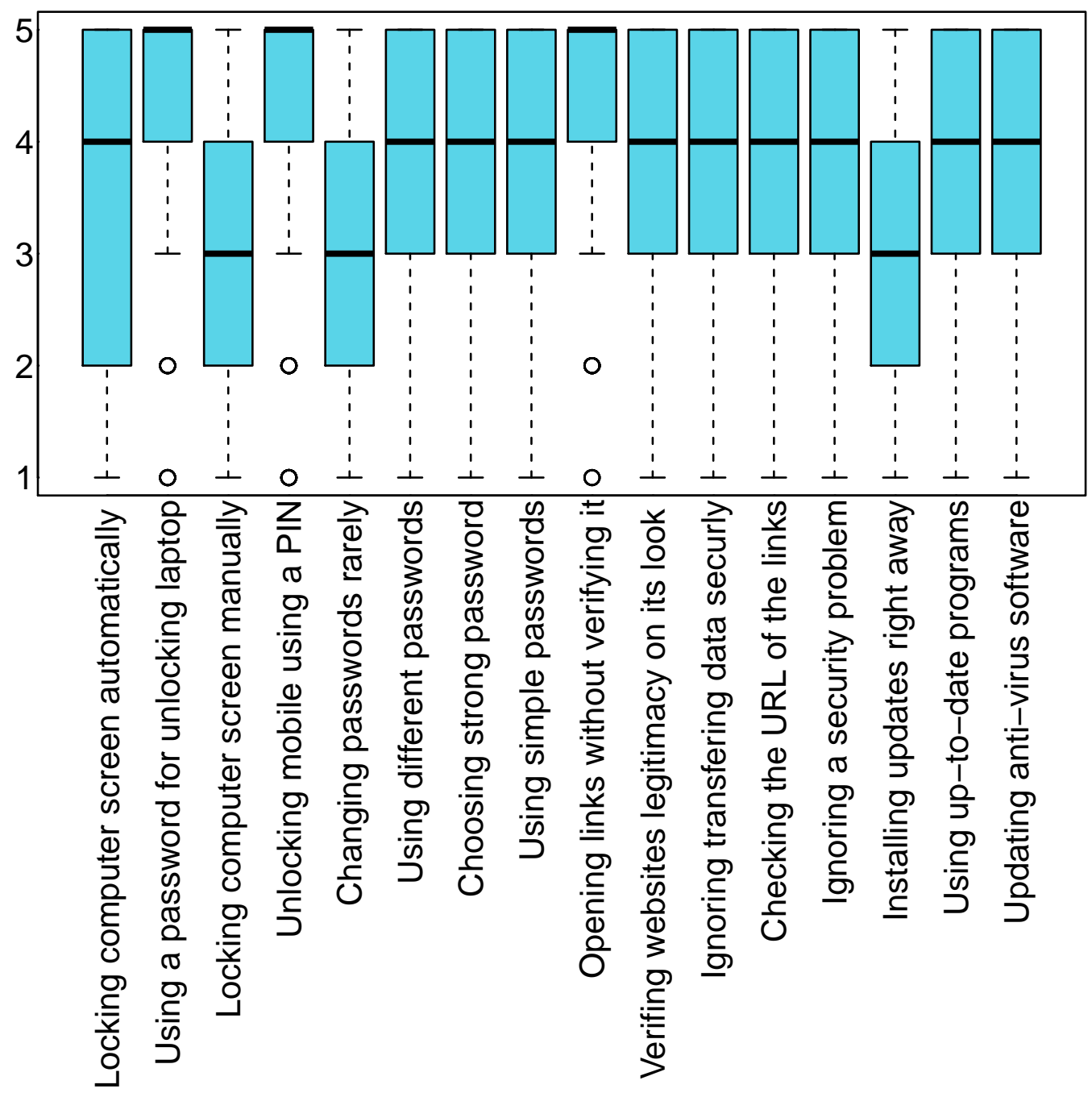

Figure 5.1: SeBIS questions 


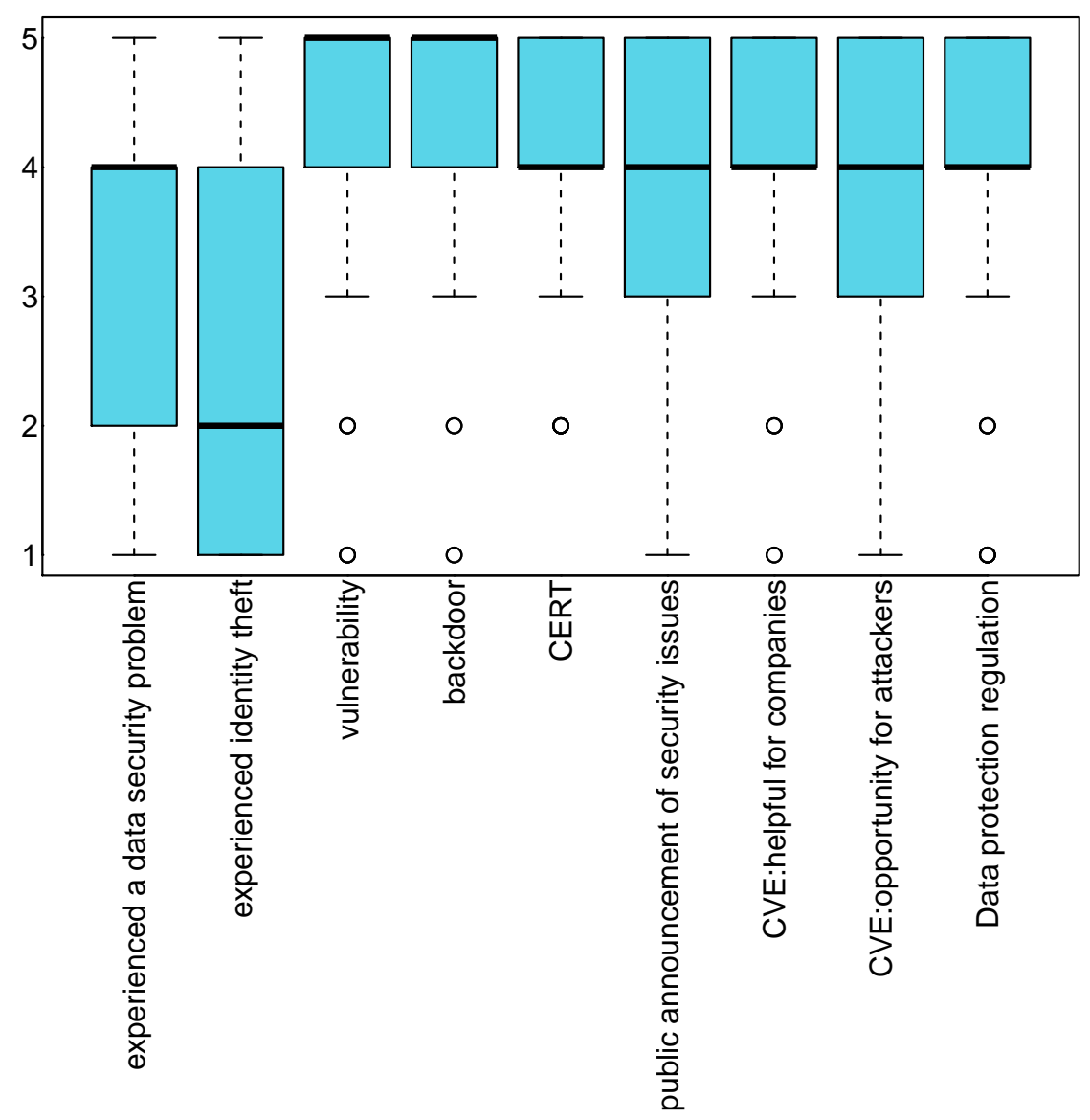

Figure 5.2: Security awareness

agreed $(4 / 5)$ that CVE is an opportunity for attackers as well as a help for companies to patch their systems' vulnerabilities.

\subsubsection{Data Breach Questions}

When we asked participants how they would rate the accountability of different people for data protection in an enterprise data breach, they rated neither high nor low for the responsibility of customers or end-users. They believed that chief information security officers (CISO) and data security operations personnel are highly responsible for a data breach, and they rated high the responsibility of CEOs, government, and CERT (see Figure 5.3).

The boxplot in Figure 5.4 shows a summary of the results of causes. Participants strongly agreed that cyber-attacks, phishing emails, unencrypted data, social 


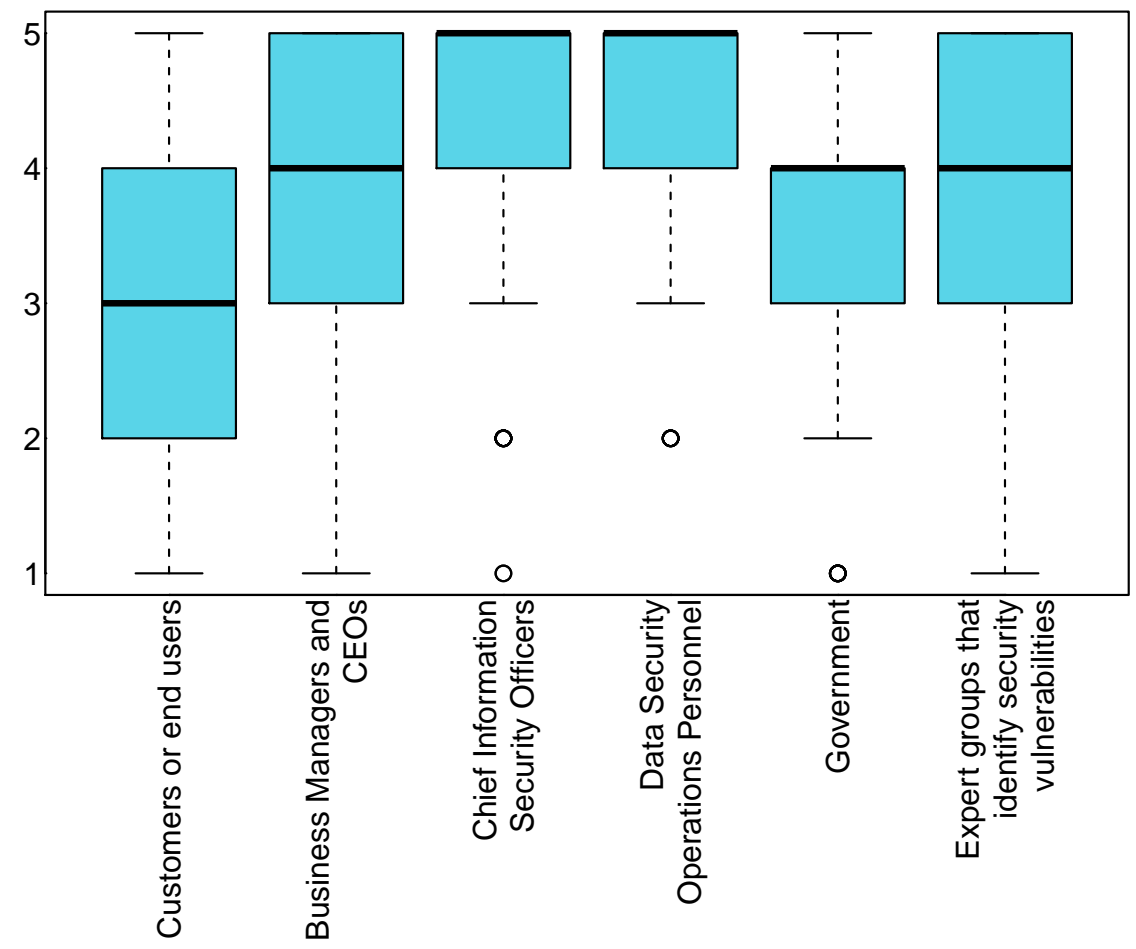

Figure 5.3: How would you rate the accountability of the following people for data protection in an enterprise data breach? 


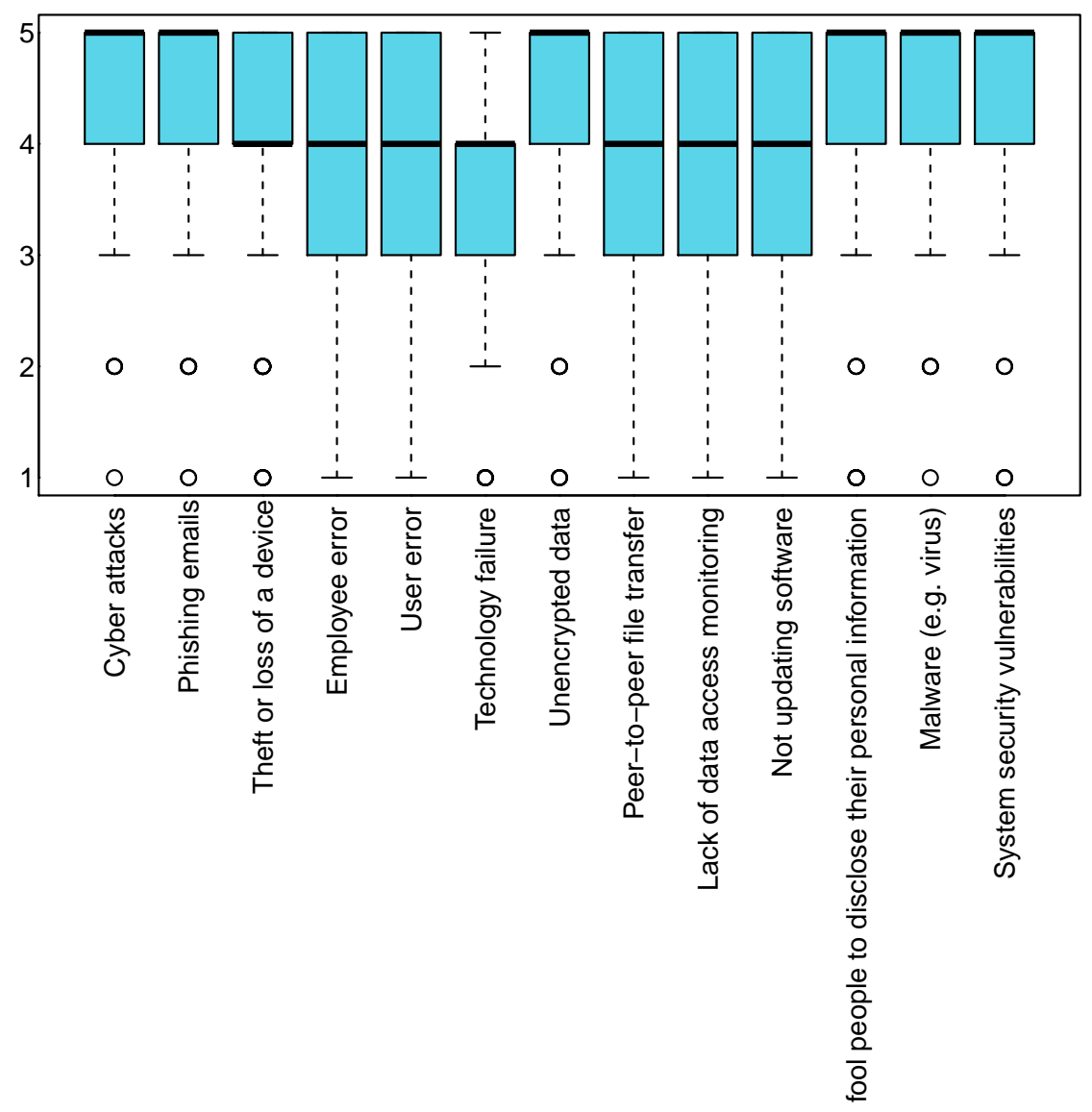

Figure 5.4: Which of the following are causes of data breach?

engineering, malware, and security vulnerabilities are the causes of a data breach $(5 / 5)$. They agreed that employee error, user error, technology failure, peer-to-peer file transfer, lack of data access monitoring, and not updating software were also the causes(4/5). When asked what can happen as a consequence of an Internet data breach, everyone agreed that invasion of privacy is the main consequence of a data breach $(5 / 5)$. They agreed that credit card fraud, identity theft, financial loss are also the consequences $(4 / 5)$. They were not quite sure if blackmail and personal safety are consequences of a data breach (see Figure 5.5.

In response to being asked what purpose is behind a data breach, participants strongly agreed that financial gain and stealing valuable intellectual property are the main reasons $(M=5 / 5)$. They agreed $(4 / 5)$ a data breach can happen to harm others, to damage the corporate reputation, for fun, for spying purposes, or political purposes 


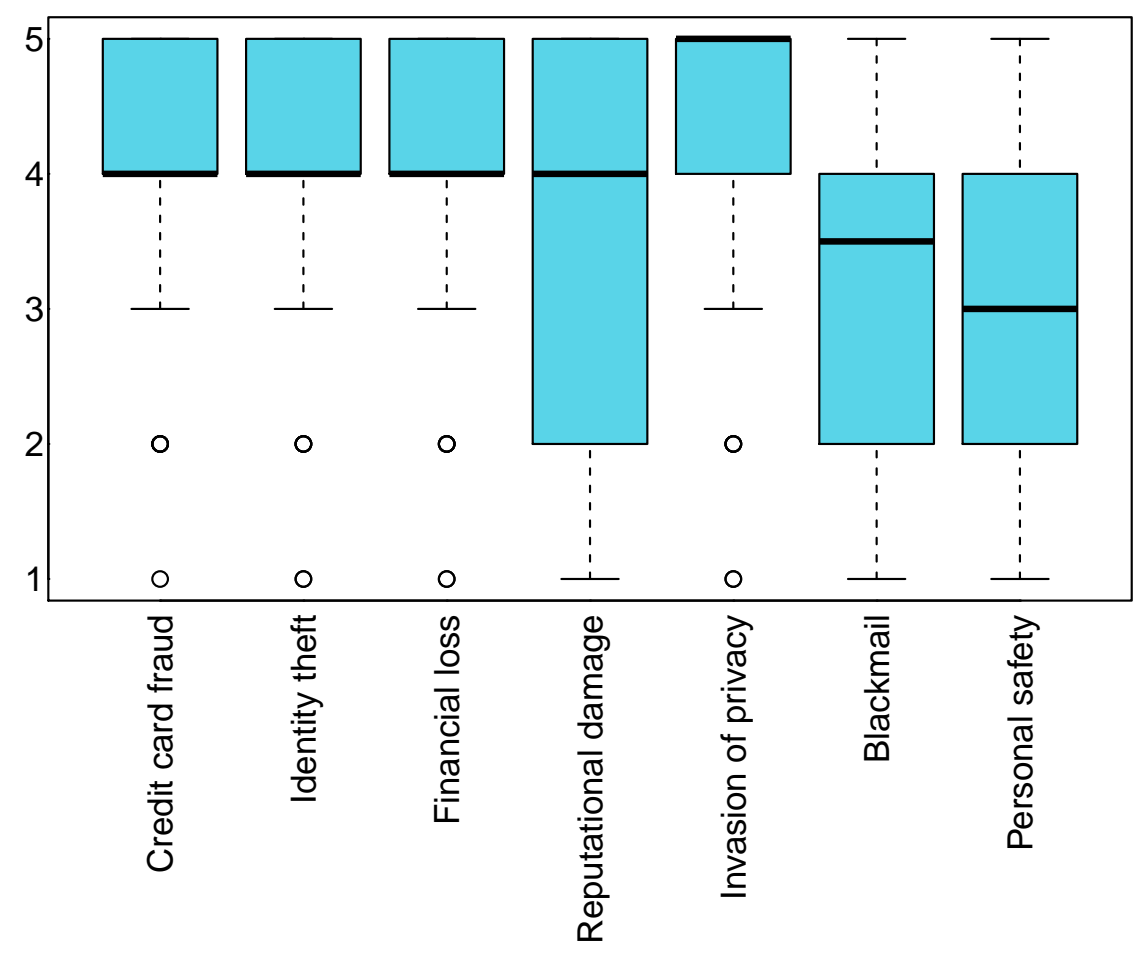

Figure 5.5: What are the consequences of a data breach? 


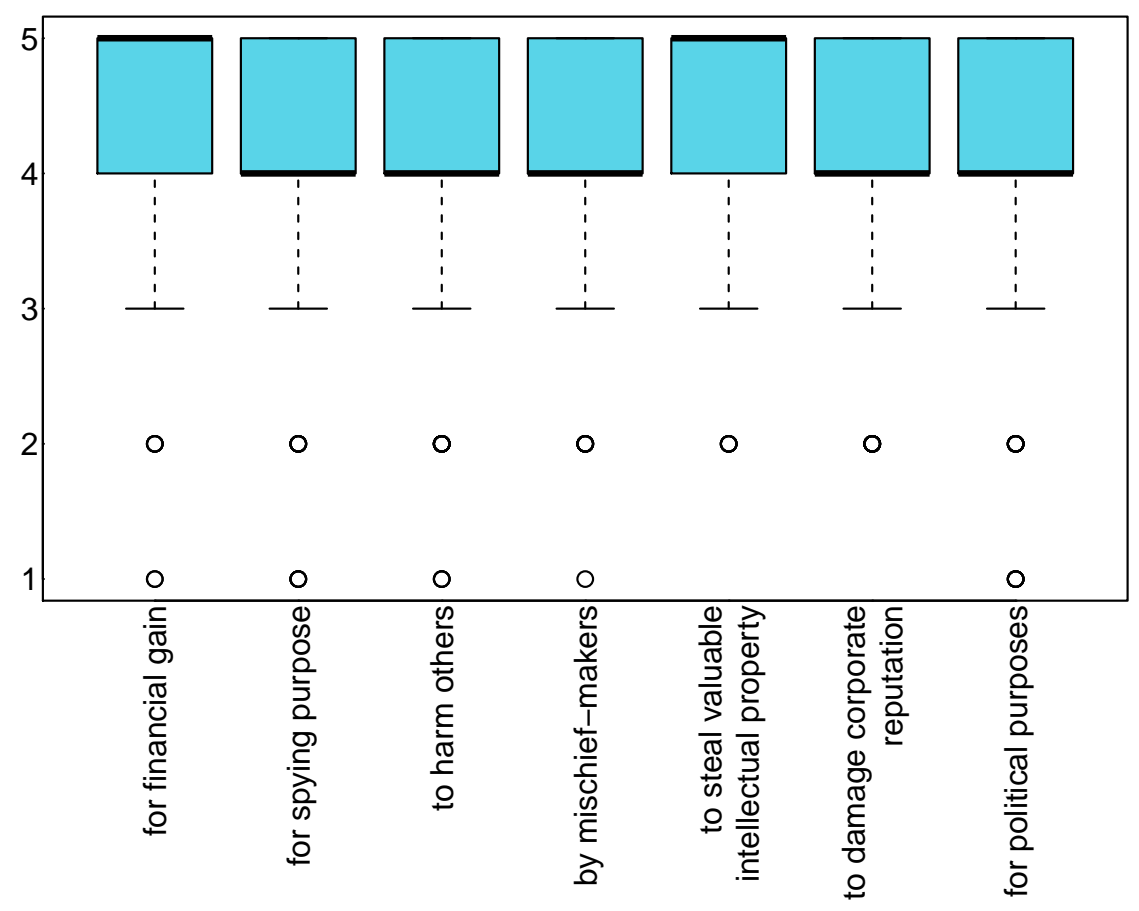

Figure 5.6: Why do data breaches happen?

(see Figure 5.6). When asked about the prevention methods, most participants were sure $(5 / 5)$ that using encryption, and multi-factor authentication can prevent a data breach. Participants were also sure $(4 / 5)$ that it is very likely to prevent a data breach by the following practices: keeping the software and hardware updated, fixing or improving all the software, restricting data retention, more strict policy, restricting user authorization and accessibility, regular audit and assessment, using intrusion detection systems. Participants were unsure if issuing new legislation can prevent a data breach (see Figure 5.7).

\subsubsection{Data Breach Description}

The box plots in Figure 5.8 shows a summary of the results of the data breach descriptions. After reading the companies press release and the news, in response to being asked who is the victim in an Internet data breach, participants strongly agreed that customers of the company are the victims $(M=5 / 5)$. However, the result for these two texts was quite different when asked if the company is the victim of the data breach. After reading the company's description of the breach, participants 


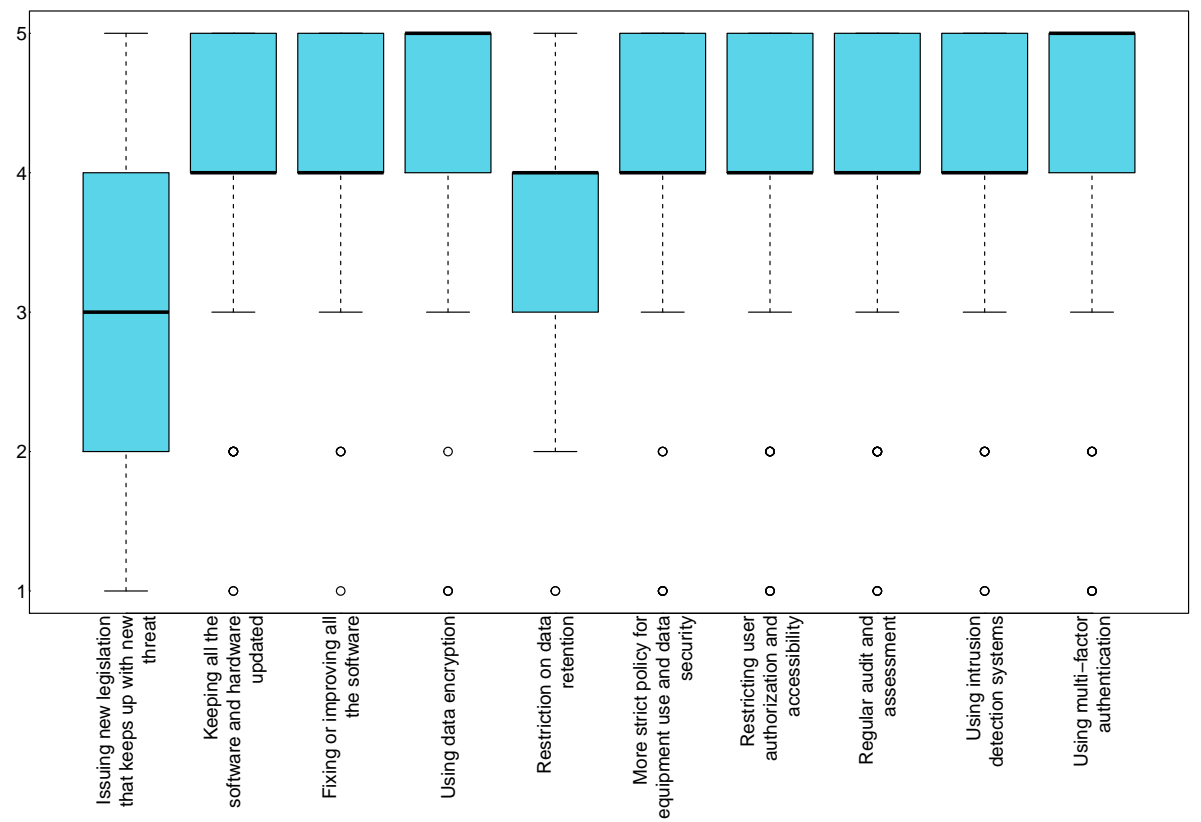

Figure 5.7: How likely would the following practices prevent a data breach?

agreed $(4.5 / 5)$ that the company is also a victim, but reading the news shows a slight different result, and they again agreed that the company could also be a victim $(4 / 5)$, the data are not normal and are skewed to the neither agreed nor disagreed.

When asked about the company's attitude regarding data protection, a considerable difference can be seen between the two texts. After reading the company's description, participants disagreed $(2 / 5)$ that the company had a relaxed attitude about protecting customers data, they also agreed that the company took security measures seriously $(4 / 5)$. However, reading the news had different results, participants agreed $(4 / 5)$ that the company had a relaxed attitude and they disagreed that the company took security measures seriously $(2 / 5)$. In response to being asked what was the attackers' purpose, we got quite similar results. They agreed that attackers wanted to harm customers as well as the company $(4 / 5)$.

After reading the company's descriptions, most participants strongly agreed (5/5) that the company was helping customers to recover from the breach. They somewhat agreed $(\mathrm{M}=4 / 5$, non normal distribution, data are skewed to disagreement) that the company put customers at risk by neglecting data protection. After reading the news, they strongly agreed that the company put the customer at risk $(5 / 5)$, and they disagreed that the company is helpful in after breach actions $(2 / 5)$. 
Table 5.4: Wilcoxon test on data breach descriptions (company vs news)

\begin{tabular}{|l|l|}
\hline Statement & P-value \\
\hline 1. The customers of the company are victims of the data breach. & $\mathrm{NS}$ \\
\hline 2. The company is the victim of the data breach. & $\mathrm{p}<0.001$ \\
\hline 3. The company had a relaxed attitude about protecting customers data. & $\mathrm{p}<0.001$ \\
\hline 4. The company takes security measures seriously. & $\mathrm{p}<0.001$ \\
\hline 5. Attackers wanted to harm the company. & $\mathrm{NS}$ \\
\hline 6. Attackers wanted to harm the customers. & $\mathrm{NS}$ \\
\hline 7. The company is helping customers recover from the breach. & $\mathrm{p}<0.001$ \\
\hline 8. The company put customers at risk by neglecting data protection. & $\mathrm{p}<0.001$ \\
\hline $\begin{array}{l}\text { 9. The company is accountable for problems resulting from the data } \\
\text { breach. }\end{array}$ & $\mathrm{NS}$ \\
\hline $\begin{array}{l}\text { 10. The customers are accountable for problems resulting from the data } \\
\text { breach. }\end{array}$ & $\mathrm{NS}$ \\
\hline
\end{tabular}

In response to being asked about the accountability, the results of reading both the company and news' text were quite similar. Most participants believed that the company is accountable for problems resulting from the data breach $(5 / 5)$, and the customers are not responsible $(2 / 5)$.

In our hypothesis, we claimed that there will be a different results when reading the company's and the news description of a data breach. To test our hypothesis, we did a Wilcoxon test to find in which pair of questions we can see a significant difference (Table 5.4). We can see that the company's and the news description make a significant difference in participants responses about victimization, company's security measures, its attitude in data protection, and helpfulness in after-breach actions (It is also marked as a red box in Figure 5.8).

We had three groups of data breach descriptions, one of these groups assigned randomly to each participants. Within each group, there was a short text from companies news releases and one from news (two sources), the order of texts appearing in each group was also randomized. We used an ANOVA test to see if the group, source, and the order affect the participants responses. As we can see in Table 5.5. the sources (company or news) make the differences and order of showing the text to participants does not affect their responses to the follow-up questions. However, the group of text affects the participants responses in whether the customers afterbreach action are helpful or not. We think it is due to the level of details about the 


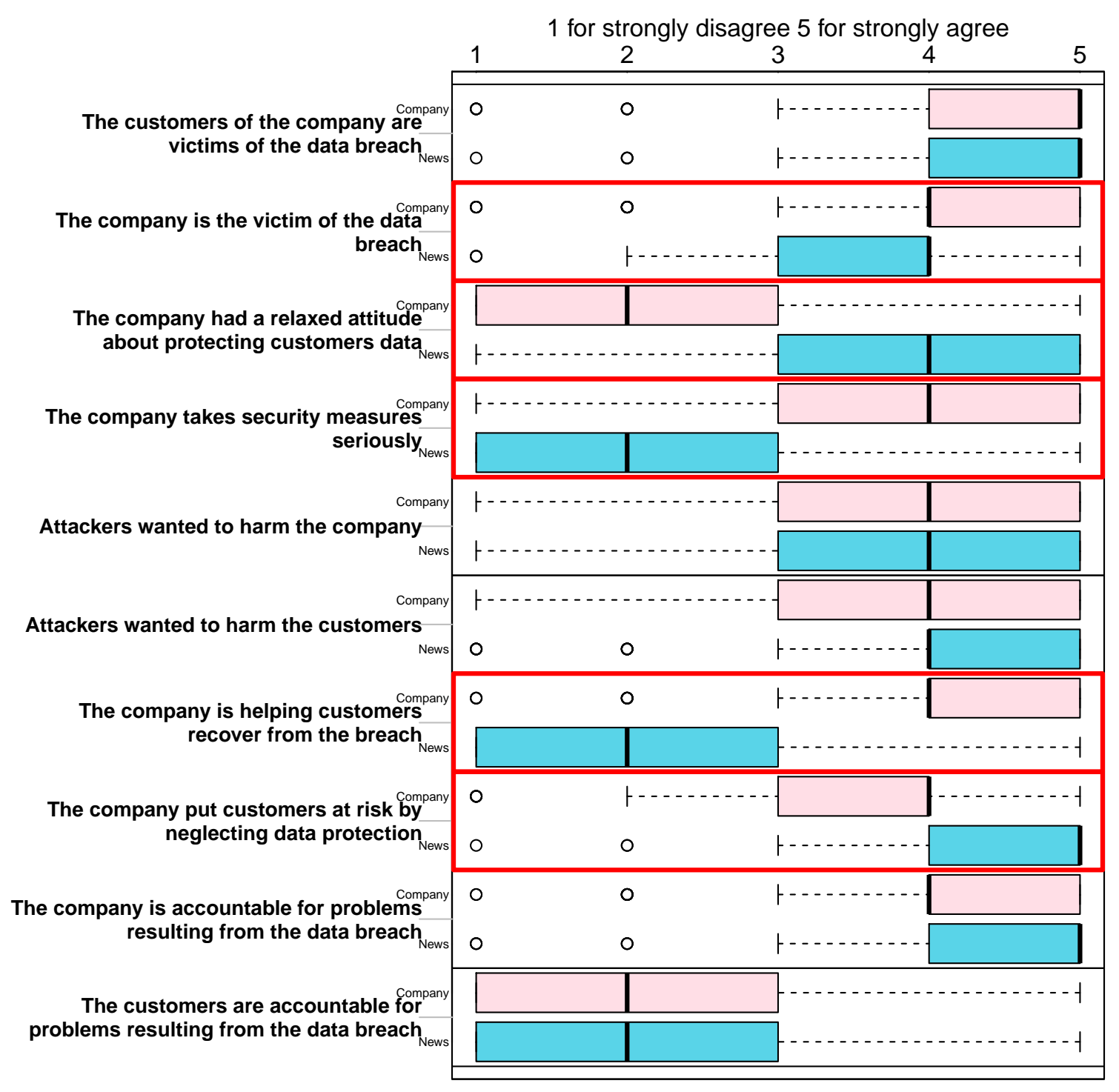

Figure 5.8: Data breach description (pink $=$ Company, blue $=$ News, red boxes $=$ Where the results are significantly different between Company and News) 
Table 5.5: ANOVA test on the data breach description

\begin{tabular}{|l|l|l|l|}
\hline Statement & \multicolumn{3}{|l|}{ P-value } \\
\hline $\begin{array}{l}\text { 1. The customers of the company are victims of } \\
\text { the data breach. }\end{array}$ & NS & NS & NS \\
\hline 2. The company is the victim of the data breach. & NS & $\mathrm{p}<0.001$ & NS \\
\hline $\begin{array}{l}\text { 3. The company had a relaxed attitude about pro- } \\
\text { tecting customers data. }\end{array}$ & $\mathrm{NS}$ & $\mathrm{p}<0.001$ & $\mathrm{NS}$ \\
\hline 4. The company takes security measures seriously. & $\mathrm{NS}$ & $\mathrm{p}<0.001$ & $\mathrm{NS}$ \\
\hline 5. Attackers wanted to harm the company. & $\mathrm{NS}$ & $\mathrm{NS}$ & $\mathrm{NS}$ \\
\hline 6. Attackers wanted to harm the customers. & $\mathrm{NS}$ & $\mathrm{NS}$ & $\mathrm{NS}$ \\
\hline $\begin{array}{l}\text { 7. The company is helping customers recover from } \\
\text { the breach. }\end{array}$ & $\mathrm{p}<0.001$ & $\mathrm{p}<0.001$ & $\mathrm{NS}$ \\
\hline $\begin{array}{l}\text { 8. The company put customers at risk by neglect- } \\
\text { ing data protection. }\end{array}$ & $\mathrm{NS}$ & $\mathrm{p}<0.001$ & $\mathrm{NS}$ \\
\hline $\begin{array}{l}\text { 9. The company is accountable for problems re- } \\
\text { sulting from the data breach. }\end{array}$ & $\mathrm{NS}$ & $\mathrm{NS}$ & $\mathrm{NS}$ \\
\hline $\begin{array}{l}\text { 10. The customers are accountable for problems } \\
\text { resulting from the data breach. }\end{array}$ & $\mathrm{NS}$ & $\mathrm{NS}$ & $\mathrm{NS}$ \\
\hline
\end{tabular}

company's after-breach actions in each group.

\subsection{Discussion}

The primary goal of this study was to explore more in details the results in study 1 that were not clear enough, and we wanted to test the generality of our results. We were also interested in finding out if the strategies used in companies after breach notification and the news about the breaches influenced people's understanding of the incident.

The first research question in this study asked what the users level of awareness in security is. The results are not consistent with our previous findings from study 1 in that the participants reported a good knowledge of vulnerability, backdoor, CERT, GDPR, CVE. As we discussed in study 1, a few people illustrated vulnerability in their drawing task as an open door or a broken wall and they mostly refer it to the vulnerable users. However, in study 3 , they rated very high when asking about their security awareness. 
The second research question in this study asked how they would rate the accountability, the causes, consequences, motivations, and prevention methods. The results of the accountability were different from study 1; it shows that participants rated the accountability of the chief information security officers and data security operations personnel very high in a data breach. The business managers and CEOs, CERT, and government are accountable in the second place, and they were not sure if the customers or end-user are accountable or not. Whereas in study 1, participants believed that they are responsible for data protection. The results of other questions were quite similar, and participants reported answers consistent with knowledge of breaches when identifying causes, consequences, motivations, and prevention methods. Based on the average time of survey and number of questions, we do not think that they had a chance to find the answers online.

We speculate that these inconsistencies between the results of study 1 and study 3 are because Mechanical Turk workers know more about security and behave more securely. They need to be more vigilant; it is recommended to secure their computer with the latest operating system security updates and virus protection software, update their browser and plug-ins with the latest versions, and use caution when directed to other websites or asked to download software. However, the histograms in Figure 5.9 and Figure 5.10 shows the differences in the participants security behavior. We conducted a one sided t-test, and we found that they are significantly different $(\mathrm{t}(28.1)=-2.19$, $\mathrm{p}$-value $=0.018)$. Moreover, we explored the effect of their level of education, and field of study on their awareness test score. However, we could not find any visible pattern to explain the reasons why the participants of study 3 have a more articulated model of Internet data breaches than the participants of study 1.

The third question in this study asked how the strategies used in the company press releases might influence the public understanding of data breaches. The result of the data breach description shows that the differences between press releases, major newspaper, and technical news when reporting the same data breach incident affect the readers' perception of the incident. After reading the text extracted from the companies press release, participants tended to rate higher the companies after breach action and security measures. They also thought that the companies were helping their customers and did not put them at any risk. However, we got different 


\section{Study1 :Histogram of SeBIS}

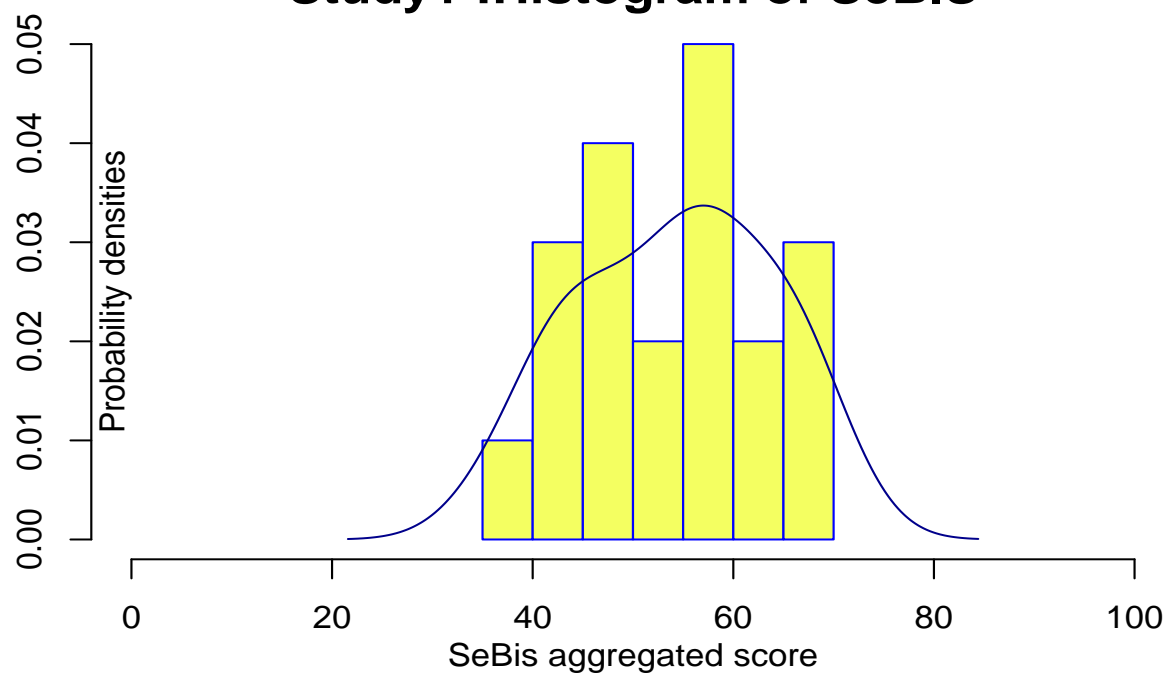

Figure 5.9: SeBIS aggregated score, Mean=54.2, $\mathrm{Sd}=9.7$

\section{Study3 :Histogram of SeBIS}

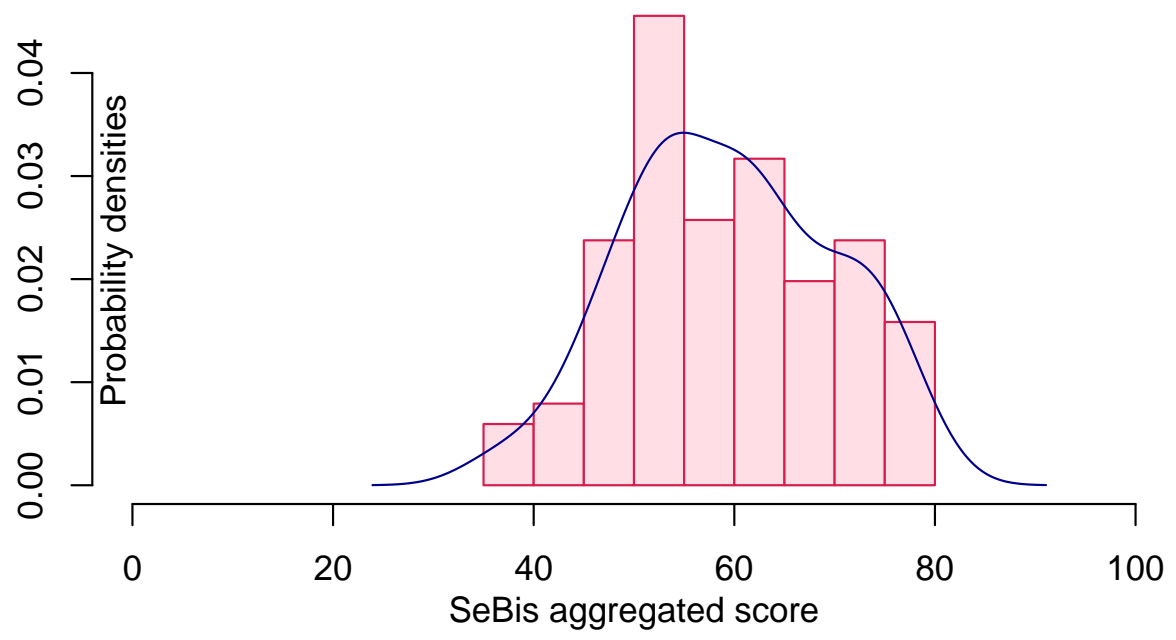

Figure 5.10: SeBIS aggregated score, Mean=59.47, $\mathrm{Sd}=10.3$ 
results when participants read the news side reporting the same incident; participants disagreed that companies took the security seriously and their after breach protective actions were not acceptable to help the customers. The companies were regarded as a victim after reading the companies description; however, the news approach in narrating the data breach resulted in a different perception of participants.

\subsection{Conclusion}

In this chapter, we presented our findings drawn from 100 participants with participants about their perception of a data breach, and the effect of different documents extracted from different sources on their perception of them.

Our results from this study suggested that users recruited from TurkPrime have more knowledge of data breaches, accountability for data protection, and security awareness. This could be due to the need to have more advanced awareness of security problem to be safe while having an online payment. Another reason could be the limitation of online survey comparing to in lab sessions that leads into less accurate data in this study. Our analysis also revealed that the dissimilar approach detected in study 2 when narrating the same story from a different point of view (companies and news) has a considerable influence on the general public's perception of a data breach incident. 


\section{Chapter 6}

\section{Conclusions}

This thesis is about exploring users' understanding of data breaches. Data breaches are a serious threat to companies as well as individuals. As computer attacks are becoming increasingly common, concern increases for people on how to stay safe online. In this thesis, we explored human factors and technologies related to data breaches. We first studied users knowledge of data breaches using questionnaires, open questions, and drawing tasks. We explored how they conceptualize the process of a data breach and how they think the attackers can access their data online. We then analyzed the documents for data breach notifications and news. We examined crisis communication strategies and evaluated framed crisis situations which are constructed by the company press releases. In our third study, we presented a quantitative study of how 100 participants from Canada and United States perceived the responsibility for data protection, causes, consequences, motivations, and preventive methods of a data breach. We also examined the way different descriptions on data breaches affect their perception and mental model of a data breach.

\subsection{Main Findings}

\subsubsection{Study 1: Exploring Mental Models of Internet Data Breaches}

From our first study, we found that participants had a basic model of Internet data breaches. They have a simple model of how an attacker can access to their data. Their mental model of data access coincides with the category of network attacks against confidentiality [80]. They understood that an attacker can capture their credentials using screen capturing or input capturing. Participants had a flawed model of what a vulnerability is, and they refer to the user who can be vulnerable due to the lack of awareness and knowledge, only a few of them has an understanding of vulnerability in a system. Participants responses for after-breach necessary actions made it evident 
that they are concerned about checking their bank transactions and changing their passwords. Although identity crime (identity theft, scam, fraud,...) is one of the most important potential consequences of a data breach, our participants did not know if there is any way to monitor or stop it. The use of people's social security numbers can cause different forms of identity theft, so the users must know what to look for and what to do if they are a victim [2]. Our research highlights the users perception of accountability for data protection; our participants believed that everyone is responsible for data protection, especially the end-users.

The results from this study show an intrinsic exigence for education of users on the possible causes of unintentional disclosure of information, and keeping them aware of prevention methods for the external attacks. Our research findings also point to an opportunity to improve users awareness on available privacy and security tools and features, so that they would be able to expect good security measures from the companies which they are sharing their information. They also should know how to reduces risk of a data breach, like how to place a credit freeze, and how to respond to a breach depending on the type of information that was compromised.

\subsubsection{Study 2: Crisis Communication Analysis}

The primary goal of this study was to examine how online news and corporate press releases reported the two largest data breach crises (Equifax and Marriott Hotels data breaches). We explored the corporate communication strategies used in their news releases. We found that Equifax and Marriott Hotels used a full range of response strategies including denial, bolstering, shift the blame, minimization, and corrective actions to reduce their reputational damage and financial loss. Using the NarrativeSemiotic method [64], this study then detected the dissimilar approach by which media and the breached companies framed the crises. The companies tried to frame the importance of security measures as severely for the company and emphasize their after-breach actions as helpful. Whereas, the news framed damage of the crisis as more severe and emphasized the poor management of the crisis by the companies, they also magnified the negative outcomes (e.g. news mentioned negative connotations like: "Equifax's Maddening Unaccountability", "Marriott's Starwood hotels mega-hack: Half a BILLION guests' deets exposed over 4 years", "Marriott Breach 
Exposes Far More Than Just Data", "Seriously, Equifax? This Is a Breach No One Should Get Away With") which is consistent with previous findings in that the news and corporate reframed the crisis differently [49, 11, 73].

\subsubsection{Study 3: Quantitative study on Internet Data Breaches}

In this study, we found that users have a more articulated models of a data breach than was showing in study 1 . They are aware of causes, consequences, motivations, and prevention methods of a data breach. They had a quite different opinion about responsibility for data protection; they were unsure if an end user is responsible for data protection in an enterprise data breach, but they rated high responsibility for the company, government, and CERT groups. We also detected that the strategies used in the corporate news releases and the media has a noticeable influence on the public understanding of data breaches. Participants responses after reading the companies description about the data breach was in line with the companies intention in showing that they are concerned about security measures and did their best to prevent the data breach. However, they responded differently after reading the news description of the data breach and this suggest the their responses was influenced by the news framing of the incident and deflection of the companies.

\subsubsection{Combining Insight and implications}

Our results suggested that participants' mental models of an Internet data breach can be basic with less detail, or more articulated. People rated high the likert type questions regarding the Internet data breach process, causes, consequences, and prevention methods. However when we asked them to describe the aforementioned elements in a data breach, they shows a lack of knowledge and uncertainty. Our studies also showed that people are not familiar with follow-up steps after a data breach, and how they can recover from identity crimes; they also regard vulnerability as lack of the users knowledge. It seems that they are not quite sure what the role of CERTs is because they agreed that CERTs are also responsible for data protection.

One pattern that emerged from our studies was the influence of breach news on the users' perception of victimization, attitudes in data protection, and responsibility. Under the GDPR regulations, it is businesses responsibility to keep personal data 
safe and protected against the unauthorized access. We illustrated two case studies in which companies failed to use appropriate technical or organisational measures that led to a massive loss of customers' personal data. These two companies were more focused on repairing their reputation and reducing their financial loss rather than protecting their customers and being transparent in describing what they know about the breach in their after-breach communications.

These results show an exigence for giving vital information about how to reduce the risk of a data breach and what steps the victims of a data breach should take to protect themselves and how to recover from the consequences. We argue that companies should explain in detail in their after-breach notifications some information about appropriate follow-up steps, and how to recover from the consequences of a data breach. In addition to awareness, users might expect the companies to offer free and usable protection mechanisms to remove any potential barrier to following protection mechanisms.

Organizations should be transparent about their data collection and data processing. They should state the purpose of data collection, storage time, and other parties who have access to data. In this case organizations should only collect data that are needed and customers will be aware of the parties who have access to their data.

There should be a technical regulations documented for companies that lay down minimum security measures and secure data processing. It may also be a good idea to include markings or labels describing the security processes, such have been proposed for website privacy policies [44]. So that if a company's security measures do not fulfill the requirements of the technical regulation, it will be considered accountable for a data breach and government should be responsible for further penalties and punishments. We suggest that users should be helped to developed awareness of security practices and regulations in order to determine where the accountability lies.

\subsection{Limitations}

Our work has certain limitations. First, our sample size for our first study can not support conclusions about the general population; when we decided to explore the generality of the results in the study 3, we ended up with slightly different results. Moreover, we did not explore some patterns that were found in the first study, such as 
whether users know about protective mechanism, and how they define a data breach. In our first study, we clearly mentioned in our recruitment poster that the study is about Internet data breaches, so participants might search about it before coming to the study session. In our online survey, they might also have a chance to search the concepts online.

Second, the nature of our two studies exploring mental models of data breaches were different. We suspect the questionnaire did not probe as deeply as the in-lab study; for example in the first study users shows less knowledge in general about data breaches, but in our survey study, participants reported a good understanding of the data breach terms like backdoor, vulnerability, and other elements like cause, and motivations. They rated high in most of our Likert type questions, and that might be because it was all the security terms that they might not be familiar with, so they just agreed in all of them without really understanding those terms. The collected data was also self-reported and qualitative in nature so our findings are subject to interpretation, and other researchers may come to different conclusion. All these points will affect the internal validity of our studies.

Our sample in study 1 consisted entirely of residents of the Canada, and in study 3 they were mostly residents of the United States, and results may be affected by their culture and may also be influenced by people's perceptions and behaviors. Our sample in study 1 and 3 skewed to younger people with higher level of education, and it is possible that this had an effect on our findings, and it might not reflect the general population. These points affect therefore the external validity of our studies.

\subsection{Contributions}

Our main contributions from this thesis are as follows:

- We identified a user's basic model of data breaches from literature.

- We conducted a study and found the results for the users' mental model of Internet data breaches.

- We conducted a text analysis to identify the strategies used in companies after breach notifications and their differences with the news. 
- We conducted a third study to find the generality of the users' mental model and also found the different factors in after-breach notifications that affect users' understanding of a data breach incident.

\subsection{Future Work}

Future work should investigate more of the factors that influence users perception of an Internet data breach. Also, a study should be conducted to create an expert mental model. Once created, it should be reviewed by technical expert, then it can be used as a baseline, in order to compare with the models' of general people and ensure its accuracy and find the knowledge gaps [70]. Another potential step can be excluding participants with some level of expertise in security, this level of expertise can be due to their work's nature or their background, to identify a more accurate model of general public. 


\section{Bibliography}

[1] Cisco. Network Attack Taxonomy. http://www.networkblue.net/cisco/ Security. Accessed: 2019-04-1.

[2] Identity theft resource center. https://www.idtheftcenter.org/ data-breaches/. Accessed: 2019-04-1.

[3] Symantec white paper - anatomy of a data breach. https://www.eval. symantec.com. Accessed: 2019-06-02.

[4] Varonis Gobal Data Risk Report 2018. https://www.varonis.com/ 2018-data-risk-report/. Accessed: 2019-05-20.

[5] Equifax's website, 2018. https://www.consumer.equifax.ca/ about-equifax/, Accessed: 2019-04-05.

[6] Marriott's website, 2018. http://www.marriott.com/default.mi, Accessed: 2019-04-05.

[7] Dominique Alessandri. Attack-Class-Based Analysis of Intrusion Detection Systems. PhD thesis, University of Newcastle upon Tyne, UK, University of Newcastle upon Tyne, 5 2004. PhD thesis.

[8] Farzaneh Asgharpour, Debin Liu, and L. Jean Camp. Mental models of security risks. In Sven Dietrich and Rachna Dhamija, editors, Financial Cryptography and Data Security, pages 367-377, Berlin, Heidelberg, 2007. Springer Berlin Heidelberg.

[9] Stefan Axelsson. Intrusion detection systems: A survey and taxonomy. Technical report, Chalmers University of Technology, 2000.

[10] William L. Benoit. Image repair discourse and crisis communication. Public Relations Review, 23(2):177 - 186, 1997.

[11] Shannon A. Bowen and Yue Zheng. Auto recall crisis, framing, and ethical response: Toyota's missteps. Public Relations Review, 41(1):40 - 49, 2015.

[12] Cristian Bravo-Lillo, Lorrie Faith Cranor, Julie Downs, and Saranga Komanduri. Bridging the gap in computer security warnings: A mental model approach. IEEE Security and Privacy, 9(2):18-26, mar 2011.

[13] Markus M. Breunig, Hans-Peter Kriegel, Raymond T. Ng, and Jörg Sander. Lof: Identifying density-based local outliers. SIGMOD Rec., 29(2):93-104, May 2000. 
[14] CA technologies. Insider threat report, 2018. https://www.ca.com/content/ $\mathrm{dam} / \mathrm{ca} / \mathrm{us} / \mathrm{files} /$ ebook/insider-threat-report.pdf, Accessed: 2019-07-13.

[15] L. J. Camp. Mental models of privacy and security. IEEE Technology and Society Magazine, 28(3):37-46, Fall 2009.

[16] Curtis A. Carver. Adaptive Agent-Based Intrusion Response. PhD thesis, Departement of Computer Science, Texas A\&M University, College Station, TX, 2001.

[17] Michelene T. H. Chi. Laboratory Methods for Assessing Experts' and Novices' Knowledge, page 167-184. Cambridge Handbooks in Psychology. Cambridge University Press, 2006.

[18] Michelene T. H. Chi, Paul J. Feltovich, and Robert Glaser. Categorization and representation of physics problems by experts and novices*. Cognitive Science, $5(2): 121-152,1981$.

[19] Federal Trade Commission. Data breach response: A guide for business, 2019. https://www.ftc.gov/tips-advice/business-center/guidance/ data-breach-response-guide-business, Accessed: 2019-06-09.

[20] GDPR compliance. Everything you need to know about GDPR compliance. https://gdpr.eu/compliance/, Accessed: 2019-08-02.

[21] W.T. Coombs and S.J. Holladay. The Handbook of Crisis Communication. Handbooks in Communication and Media. Wiley, 2012.

[22] Cassandra Cross, Megan Parker, and Daniel Sansom. Media discourses surrounding 'non-ideal' victims: The case of the Ashley Madison data breach. International Review of Victimology, 25(1):53-69, 2019.

[23] Paul Barton Stephanie Tomasevic Duncan Geere David McCandless, Tom Evans. World's biggest data breaches and hacks. isources: databreaches.net, IDTheftCentre and media reports.

[24] George Dionisopoulos and S.L. Vibbert. CBS vs. Mobil Oil: Charges of creative bookkeeping in 1979. Oratorical Encounters, pages 241-251, 011988.

[25] Serge Egelman and Eyal Peer. Scaling the security wall: Developing a security behavior intentions scale (SeBIS). In Proceedings of the 33rd Annual ACM Conference on Human Factors in Computing Systems, CHI '15, pages 2873-2882, New York, NY, USA, 2015. ACM.

[26] Uwe Flick. The SAGE Handbook of Qualitative Data Analysis. Research methods in education. SAGE Publications, 2014.

[27] The European Union Agency for Cybersecurity. ENISA Threat Landscape Report 2018. ETL 2018. 
[28] Batya Friedman, David Hurley, Daniel C. Howe, Helen Nissenbaum, and Edward Felten. Users' conceptions of risks and harms on the web: A comparative study. In CHI '02 Extended Abstracts on Human Factors in Computing Systems, CHI EA '02, pages 614-615, New York, NY, USA, 2002. ACM.

[29] Namey E. Guest G., MacQueen K. M. Introduction to Applied Thematic Analysis. Applied Thematic Analysis. SAGE Publications, 2012.

[30] Simon Hansman and Ray Hunt. A taxonomy of network and computer attacks. Comput. Secur., 24(1):31-43, Feb 2005.

[31] Louis Hébert. The actantial mode, 2006. http://www.signosemio.com/ greimas/actantial-model.asp, Accessed: 2019-01-07.

[32] Andreas Wespi Hervé Debar, Marc Dacier. Towards a taxonomy of intrusiondetection systems. Computer Networks, 31(8):805-822, 4 1999. An optional note.

[33] Joahnna Marie Hipolito. Anatomy of a data breach, 2011. https: //www.trendmicro.com/vinfo/us/threat-encyclopedia/web-attack/ 110/anatomy-of-a-data-breach, Accessed: 2019-06-07.

[34] G.E. Hynes. Managerial Communication: Strategies and Applications. SAGE Publications, 2015.

[35] Alexander Jenkins, Murugan Anandarajan, and Rob D'Ovidio. 'All that Glitters is not Gold': The Role of Impression Management in Data Breach Notification. Western Journal of Communication, 78(3):337-357, 2014.

[36] Carlos Jensen and Colin Potts. Privacy policies as decision-making tools: An evaluation of online privacy notices. In Proceedings of the SIGCHI Conference on Human Factors in Computing Systems, CHI '04, pages 471-478, New York, NY, USA, 2004. ACM.

[37] Johnson-Laird. Mental models., pages 469-499. The MIT Press, Cambridge, MA, US, 1989.

[38] David Jonassen and Young Hoan Cho. Externalizing mental models with mindtools. Understanding Models for Learning and Instruction, pages 145-159, 01 2008.

[39] David Jonassen and Young Hoan Cho. Externalizing Mental Models with Mindtools, pages 145-159. Springer US, Boston, MA, 2008.

[40] David H. Jonassen. On the role of concepts in learning and instructional design. Educational Technology Research and Development, 54(2):177, May 2006.

[41] D.H. Jonassen. Computers as Mindtools for Schools: Engaging Critical Thinking. Merrill, 2000. 
[42] Russell K. Schutt Joseph Check. Qualitative data analysis. Research methods in education. SAGE Publications, 2012.

[43] Ruogu Kang, Laura Dabbish, Nathaniel Fruchter, and Sara Kiesler. "My Data Just Goes Everywhere:" User Mental Models of the Internet and Implications for Privacy and Security. In Eleventh Symposium On Usable Privacy and Security (SOUPS 2015), pages 39-52, Ottawa, 2015. USENIX Association.

[44] Patrick Gage Kelley, Joanna Bresee, Lorrie Faith Cranor, and Robert W. Reeder. A "nutrition label" for privacy. In Proceedings of the 5th Symposium on Usable Privacy and Security, SOUPS '09, pages 4:1-4:12, New York, NY, USA, 2009. ACM.

[45] C. Kelly. Data security: A new concern for PR practitioners. Public Relations Quarterly, (50):25-26, 2005.

[46] Kristopher Kendall. A database of computer attacks for the evaluation of intrusion detection systems. Master's thesis, Massachussets Institute of Technology, The address of the publisher, 51999.

[47] Nitesh Kumar Jayant Shekhar Khaleel Ahmad, Shikha Verma. Classification of internet security attacks. In Proceedings of the 5th National Conference; INDIACom-2011, New Delhi, 2011.

[48] Georgia Killcrece. Steps for Creating National CSIRTs . Technical report, Carnegie Mellon University Pittsburgh PA, 082004.

[49] Bokyung Kim, Kristine Johnson, and Sun-Young Park. Lessons from the five data breaches: Analyzing framed crisis response strategies and crisis severity. Cogent Business \&s Management, 4(1), 2017.

[50] Nahyun Kim and Suman Lee. Cybersecurity breach and crisis response: An analysis of organizations' official statements in the United States and South Korea. International Journal of Business Communication, 0(0):2329488418777037, 0.

[51] Houle KJ, Weaver GM, N Long, and R Thomas. Trends in denial of service attack technology. CERT, 012001.

[52] Edwin M. Knorr and Raymond T. Ng. Algorithms for mining distance-based outliers in large datasets. In Proceedings of the 24rd International Conference on Very Large Data Bases, VLDB '98, pages 392-403, San Francisco, CA, USA, 1998. Morgan Kaufmann Publishers Inc.

[53] F. Liu L. Cheng and D. D. Yao. Enterprise data breach: Causes, challenges, prevention, and future directions. Data Mining Knowl. Discovery, 7(5):e1211, December 2018. 
[54] David L Sturges. Communicating through crisis: A strategy for organizational survival. Management Communication Quarterly - MANAG COMMUN Q, 7:297-316, 021994.

[55] Roy Langer and Signe Thorup. Building trust in times of crisis: Storytelling and change communication in an airline company. Corporate Communications: An International Journal, 11, 102006.

[56] Aleksandar Lazarevic, Levent Ertöz, Vipin Kumar, Aysel Ozgur, and Jaideep Srivastava. A comparative study of anomaly detection schemes in network intrusion detection. In SDM, 2003.

[57] Aleksandar Lazarevic and Jaideep Kumar, Vipinand Srivastava. Intrusion Detection: A Survey, pages 19-78. Springer US, Boston, MA, 2005.

[58] L.L. Leape. Apology for errors: Whose responsibility? rontiers of Health Services Management, 28(3):3-12, 2012.

[59] Suman Lee and Surin Chung. Corporate apology and crisis communication: The effect of responsibility admittance and sympathetic expression on public's anger relief. Public Relations Review, 38(5):932 - 934, 2012.

[60] Breach Index Level. Data privacy and new regulations take center stage. 2018 first half review.

[61] Abberbock T. Litman L., Robinson J. Turkprime.com: A versatile crowdsourcing data acquisition platform for the behavioral sciences. In Behavior Research Methods, pages 1-10. Springerlink.com, 2016.

[62] Mary Ellen Guffey; Dana Loewy. Business communication : process and product. Stamford, CT : Cengage Learning, 2015.

[63] Nate Lord. A TIMELINE OF THE ASHLEY MADISON HACK, 2017. https://digitalguardian.com/blog/timeline-ashley-madison-hack, Accessed: 2019-08-02.

[64] S. Marsen. Communication Studies. Palgrave Foundations Series. Macmillan Education UK, 2006.

[65] S. Marsen. "Lock the Doors": Toward a Narrative-Semiotic Approach to Organizational Crisis. Journal of Business and Technical Communication, 28(3):301$326,2014$.

[66] S. Marsen. A narrative-semiotic approach to management communication: The case of the Columbia space shuttle accident. SAGE Research Methods Cases, 2015 .

[67] Sky Marsen. A narrative-semiotic approach to management communication: The case of the columbia space shuttle accident. SAGE Publications, Sep 2015. 
[68] Edwen McAskill and Alex Hern. Edward Snowden: "The people are still powerless, but now they're aware", 2018. https://www.theguardian.com/us-news/ 2018/jun/04/edward-snowden-people-still-powerless-but-aware, Accessed: 2019-08-02.

[69] Erin McCann. Hackers exploit Heartbleed to swipe data of 4.5 million, 2014. https://www.healthcareitnews.com/news/ hackers-exploit-heartbleed-swipe-data-45-million, Accessed: 201908-02.

[70] M.G. Morgan, Fischhoff, B. Fischhoff, A. Bostrom, and C.J. Atman. Risk Communication: A Mental Models Approach. Cambridge University Press, 2002.

[71] Philip N. and Johnson-Laird. Mental models: Towards a cognitive science of language, inference, and consciousness. Cambridge University Press, 1990.

[72] Jakob Nielsen. Mental Models. https://www.nngroup.com/articles/ mental-models/, Accessed: 2019-04-10.

[73] Jos Nijkrake, Jordy F. Gosselt, and Jan M. Gutteling. Competing frames and tone in corporate communication versus media coverage during a crisis. Public Relations Review, 41(1):80 - 88, 2015.

[74] Global Forum on Cyber Expertise. Global good practices-national computer security incident response teams. Global Conference on CyberSpace 2017.

[75] S. Pontarelli, G. Bianchi, and S. Teofili. Traffic-aware design of a high-speed fpga network intrusion detection system. IEEE Transactions on Computers, 62(11):2322-2334, Nov 2013.

[76] Fahimeh Raja, Kirstie Hawkey, and Konstantin Beznosov. Revealing hidden context: Improving mental models of personal firewall users. In Proceedings of the 5th Symposium on Usable Privacy and Security, SOUPS '09, pages 1:1-1:12, New York, NY, USA, 2009. ACM.

[77] GAO Report. Data Protection: Actions Taken by Equifax and Federal Agencies in Response to the 2017 Breach GAO-18-559. 2018.

[78] GAO Report. What the next congress should do to prevent a recurrence of the equifax data breach. 2018.

[79] Derek A. Smith. 7 steps of a cyber attack and what you can do to, 2017. https://www.beyondtrust.com/blog/entry/ 7-steps-cyber-attack-can-protect-windows-privileged-accounts, Accessed: 2019-07-31.

[80] Irshad Sumra, Halabi Bin Hasbullah, and Jamalul-Lail Ab Manan. Attacks on security goals (confidentiality, integrity, availability) in vanet: A survey. volume 306, 062014 . 
[81] Symantec. ISTR: Internet Security Threat Report, 2019. https://resource. elq.symantec.com/LP=6819?inid=symc_threat-report_istr_to_leadgen_ form_LP-6819_ISTR-2019-report-main\&cid=70138000001Qv0PAAS, Accessed: 2019-07-12.

[82] ITSMF UK. ITIL Foundation Handbook. The Stationery Office, Norwich, 3rd edition, 2012.

[83] Jennifer R. Veltsos. An analysis of data breach notifications as negative news. Business Communication Quarterly, 75(2):192-207, 2012.

[84] Common Vulnerabilities and Exposures. About cve. Global Conference on CyberSpace 2017.

[85] Rick Wash. Folk models of home computer security. In Proceedings of the Sixth Symposium on Usable Privacy and Security, SOUPS '10, pages 11:1-11:16, New York, NY, USA, 2010. ACM.

[86] Rick Wash and Emilee Rader. Influencing mental models of security: A research agenda. In Proceedings of the 2011 New Security Paradigms Workshop, NSPW '11, pages 57-66, New York, NY, USA, 2011. ACM.

[87] Justin Wu and Daniel Zappala. When is a tree really a truck? exploring mental models of encryption. In Fourteenth Symposium on Usable Privacy and Security (SOUPS 2018), pages 395-409, Baltimore, MD, 2018. USENIX Association.

[88] Z. Wu, Y. Ou, and Y. Liu. A taxonomy of network and computer attacks based on responses. In 2011 International Conference of Information Technology, Computer Engineering and Management Sciences, volume 1, pages 26-29, Sep. 2011.

[89] J X. Kasperson, Roger E. Kasperson, Nick Pidgeon, and Paul Slovic. The social amplification of risk: Assessing fifteen years of research and theory. The social amplification of risk, 1, 012003.

[90] A.J. Zaremba. Crisis Communication: Theory and Practice. Taylor \& Francis Group, 2015.

[91] Yixin Zou, Abraham H. Mhaidli, Austin McCall, and Florian Schaub. "I've Got Nothing to Lose": Consumers' Risk Perceptions and Protective Actions after the Equifax Data Breach. In Fourteenth Symposium on Usable Privacy and Security (SOUPS 2018), pages 197-216, Baltimore, MD, 2018. USENIX Association. 


\section{Appendix A}

Study 1: Recruitment Poster, Consent Form, Questionnaire 


\title{
Carleton
}

U N I VER S T Y

Canada's Capital University

\section{Participate in a study on "Exploring mental models of Internet data breaches"!}

To participate in this study, you must be:

\author{
$\checkmark$ At least 18 years old \\ $\checkmark$ Comfortable in the English language
}

This is a 60 to 90 minutes study. You will be asked to do a drawing task and answer some questions.

Participants will be compensated with $\$ 20$ cash.

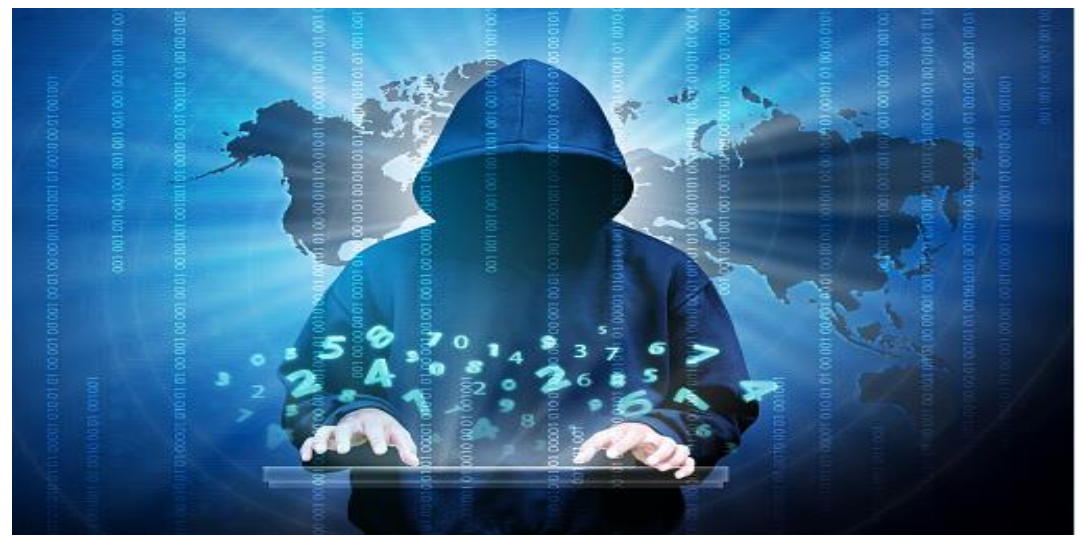

Please contact the researcher, Zahra Hassanzadeh, for more details on this study at (zahra.hassanzadeh@ carleton.ca). (Faculty supervisor: Dr. Robert Biddle ) [CUREBB, Clearance \#110780] 


\section{A.2 Research Consent Form}

\section{Carleton U N I V E R I T Y \\ Canada's Capital University}

\section{Name and Contact Information of Researchers:}

Zahra Hassanzadeh, Carleton University, School of Computer Science

Email: zahrahassanzadeh@cmail.carleton.ca

Supervisor and Contact Information:

Dr. Robert Biddle, Carleton University, School of Computer Science

Email: Robert.Biddle@carleton.ca

\section{Project Title}

Exploring Mental Models of Internet data breaches

\section{Project Sponsor and Funder (if any)}

NSERC Discovery Grant RGPIN-2016-06149 "New Directions in Usable Security"

\section{Carleton University Project Clearance}

CUREB-B Clearance \#: 110780

Date of Clearance: May 13, 2019

\section{Invitation}

You are invited to take part in a research project. The information in this form is intended to help you understand what we are asking of you so that you can decide whether you agree to participate in this study. Your participation in this study is voluntary, and a decision not to participate will not be used against you in any way. As you read this form, and decide whether to participate, please ask all the questions you might have, take whatever time you need, and consult with others as you wish.

\section{What is the purpose of the study?}

In this project, we will conduct a user study to explore users' mental model of Internet data breaches. A data breach happens when an unauthorised person accesses private or confidential information.

\section{What will I be asked to do?}

If you agree to take part in the study, we will ask you to fill out one demographic questionnaire, then you will be asked to draw a simple little picture of your perception of how a data breach happens, you will have as much time as you need. Afterwards, we will ask you to answer some open questions followed by a few closed questions. The whole session will take about 60 to 90 minutes. 


\section{Risks and Inconveniences}

We do not anticipate any risks to participating in this study.

\section{Possible Benefits}

You may not receive any direct benefit from your participation in this study. However, your participation may allow researchers to better understand users' perception of Internet data breaches and try to find ways to enhance your intuitive mental models, to better understand privacy and security issues in Internet.

\section{Compensation/Incentives}

You will be paid $\$ 20$ for your participation in this study.

\section{No waiver of your rights}

By signing this form, you are not waiving any rights or releasing the researchers from any liability.

\section{Withdrawing from the study}

You may decline to answer any question and you may withdraw from the interview at any point without providing justification. Withdrawal from the study is also allowed when re-checking with participants at the end of the study. If you choose to withdraw, your data will be destroyed. Withdrawal from the study is not possible after completing the study session as identifiers will not be kept.

\section{Confidentiality}

We will treat your personal information as confidential. None of your personal information will be accessed, nor will you be asked to disclose personally identifiable information. Research records may be accessed by the Carleton University Research Ethics Board in order to ensure continuing ethics compliance.

The results of this study will be used for a Master thesis and it may be published or presented at an academic conference or meeting later but the data will be presented so that it will not be possible to identify any participants.

You will be assigned a code so that your identity will not be associated with the data you have provided. Our questionnaires are hosted on Limesurvey installed on our research lab's server. This server is secure: it has daily anti-malware checks, is password-protected, and is located in a secure server room at 2118 HCI Building. Your data will be stored and protected by Carleton University's HotSoft research lab in the HCI Building on the Carleton campus but may be disclosed via a court order or data breach. All data, including coded information, will be kept in a password-protected computer. Only researchers directly involved in the research will have access to the study data. This data may be used for future research on a related topic. With your permission, the interview will be audio-recorded to make it easier to accurately record your thoughts and opinion. If you do not accept to be audio-recorded, we will take notes during the session. 


\section{Ethics review}

This project was reviewed and cleared by the Carleton University Research Ethics Board. If you have any ethical concerns with the study, please contact Dr. Bernadette Campbell, Chair, Carleton University Research Ethics Board-B (by phone at 613-520-2600 ext. 4085 or by email at ethics@ carleton.ca). For all other questions about the study, please contact the researcher.

\section{Statement of consent - print and sign name}

I voluntarily agree to participate in this study.

I agree to be audio recorded.

My anonymized identifier (e.g. P1, P2) can be used in

Publications/ presentations.

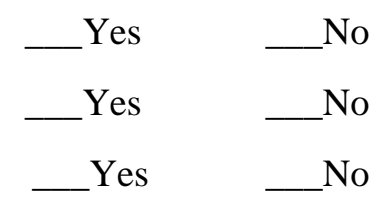

Signature of participant

Date

\section{Research team member who interacted with the subject}

I have explained the study to the participant and answered any and all of their questions. The participant appeared to understand and agree.

Signature of researcher

Date 


\section{Hello, and welcome to this survey.}

\section{Section A: Demographics Questionnaire}

Participants Demographic Survey

A1. What is your participant number?

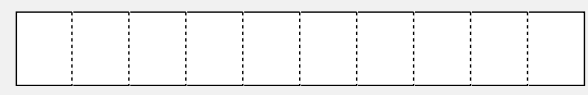

A2. Please state your age:

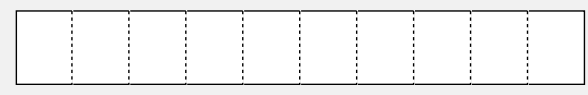

A3. Please state your gender:

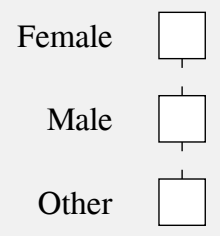

A4. What is your highest/current academic level?

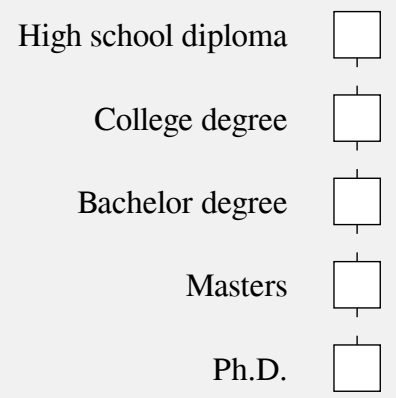

A5. What is/was your major field of study? Please state all if more than one.

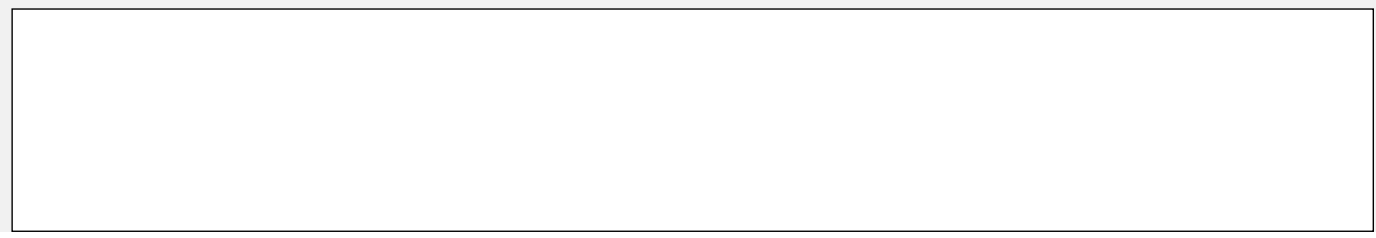

A6. To your knowledge, have you ever experienced any data security problem, like someone trying to secretly change your password?

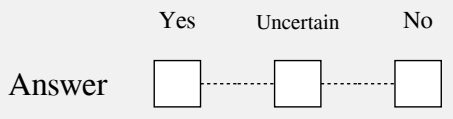


A7. If you answered yes, please describe your experience.

\section{A8. To your knowledge, have you ever experienced identity theft, like someone applying for credit card using your information?}

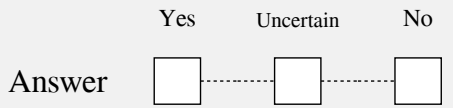

A9. If you answered yes, please describe your experience.

\section{Section B: SeBIS}

Security Behavior Intentions Scales

\section{B1. Security Behavior Intentions Scales}

I set my computer screen to automatically lock if I don't use it for a prolonged period of time.

I use a password/passcode to unlock my laptop or tablet.

I manually lock my computer screen when I step away from it.

I use a PIN or passcode to unlock my mobile phone.

I do not change my passwords, unless I have to.

I use different passwords for different accounts that I have.

When I create a new online account, I try to use a password that goes beyond the site's minimum requirements.

I do not include special characters in my password if it's not required.

When someone sends me a link, I open it without first verifying where it goes.

I know what website I'm visiting based on its look and feel, rather than by looking at the URL bar.
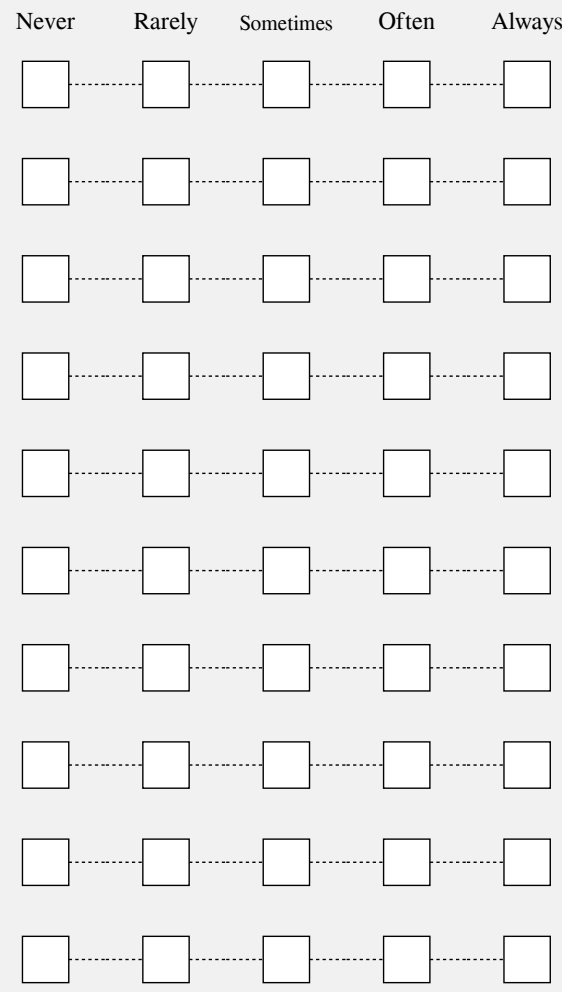


\section{|||||||||||||||||||||||||||}

I submit information to websites without first verifying that it will be sent securely (e.g., SSL, "https://", a lock icon).

When browsing websites, I mouseover links to see where they go, before clicking them.
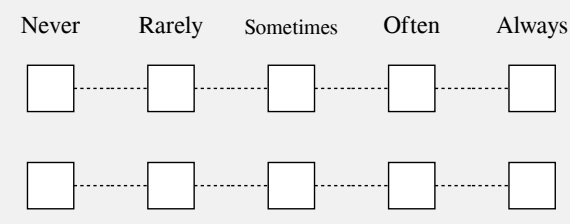

If I discover a security problem, I continue what I was doing because I assume someone else will fix it.

When I'm prompted about a software update, I install it right away.

I try to make sure that the programs I use are up-to-date.

I verify that my anti-virus software has been regularly updating

itself.
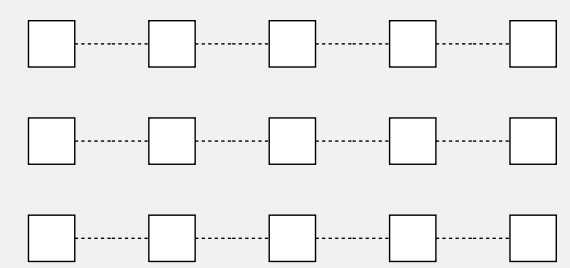

\section{Section C: Drawing Task}

The purpose of this exercise is to help us understand what you have in mind. This is not a test of artistic ability, and there are no 'correct' ways of drawing this picture.

C1. Using a paper and pencil, please draw your understanding of how a data breach happens. In other words, try to draw what is happening when it is said that personal information was exposed in a hack. (Elements you may need for this drawing: Admin, Data, Customer, Attacker, Access to data, Vulnerability)

\section{Section D: Open Questions}

D1. What is considered an Internet data breach, as you understand it?

D2. Have you ever heard of an Internet data breach?

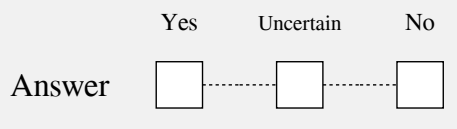

D3. If you answered yes, which one do you remember? where did you read about that? 
D4. What causes an Internet data breach?

D5. What happens in an Internet data breach?

D6. Why does Internet data breach happen? In other words, what purpose is behind an Internet data breach?

D7. How would you know if you have been affected in an Internet data breach?

D8. What can you do if your data has been breached?

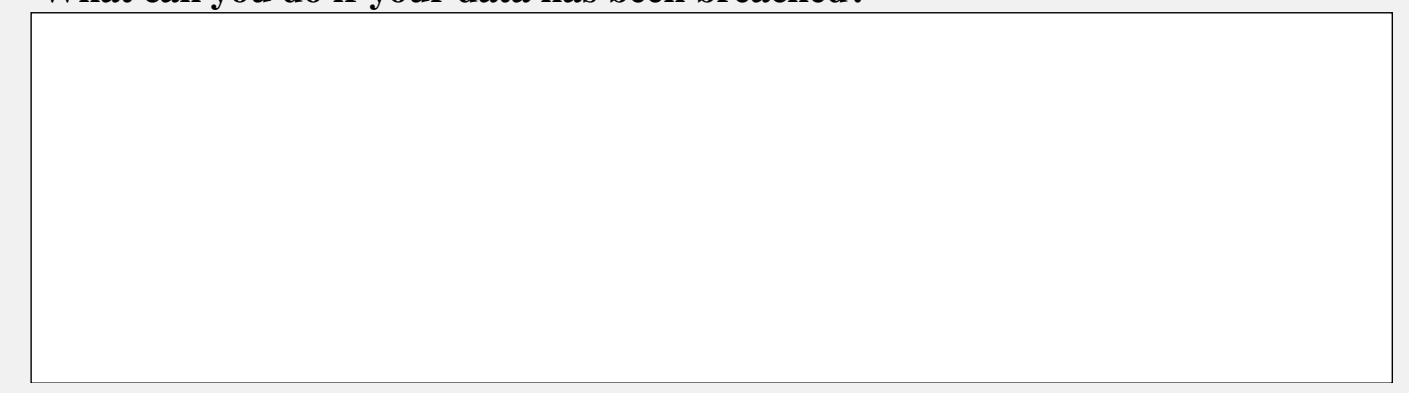


D9. What should companies do to protect your data from data breaches?

D10. How can Internet data breaches be prevented?

D11. What are the consequences of Internet data breach?

D12. Who is responsible for data protection?

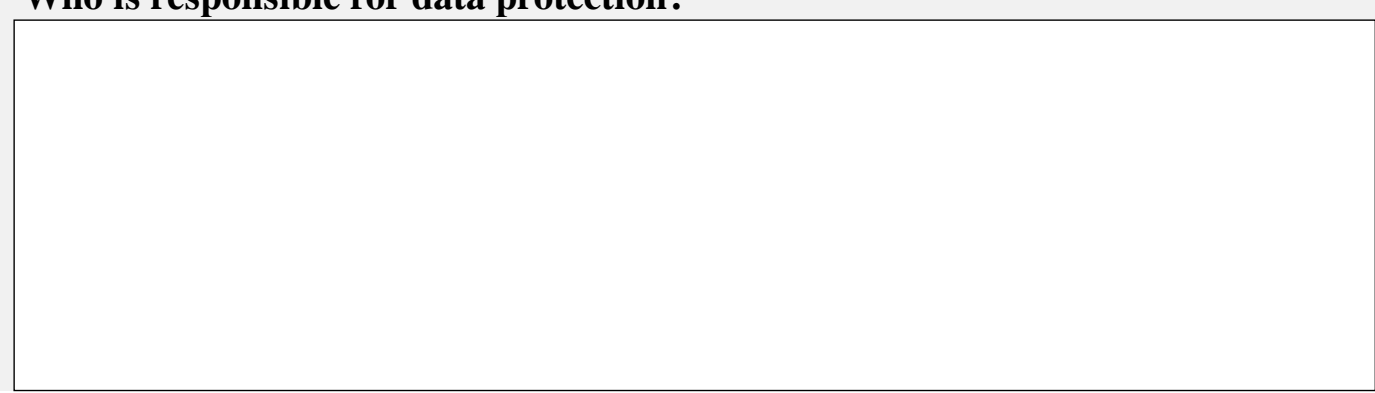

\section{Section E: Closed Questions}

E1. Which of the following are causes of data breach?

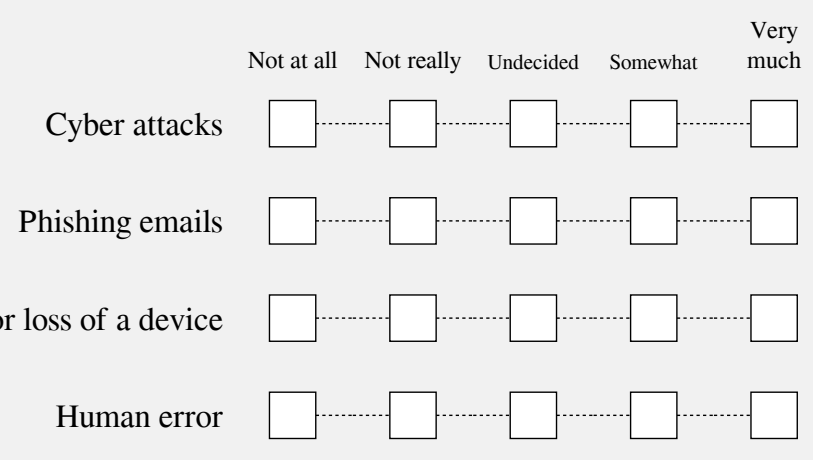




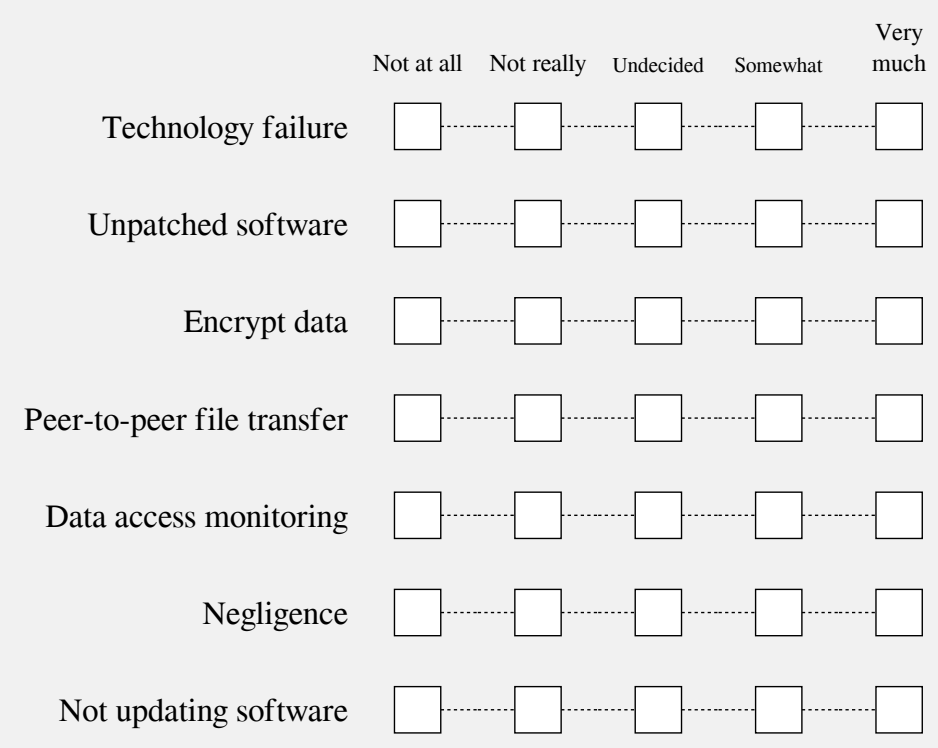

E2. How likely is that the following items happen as a consequence of an Internet data breach?

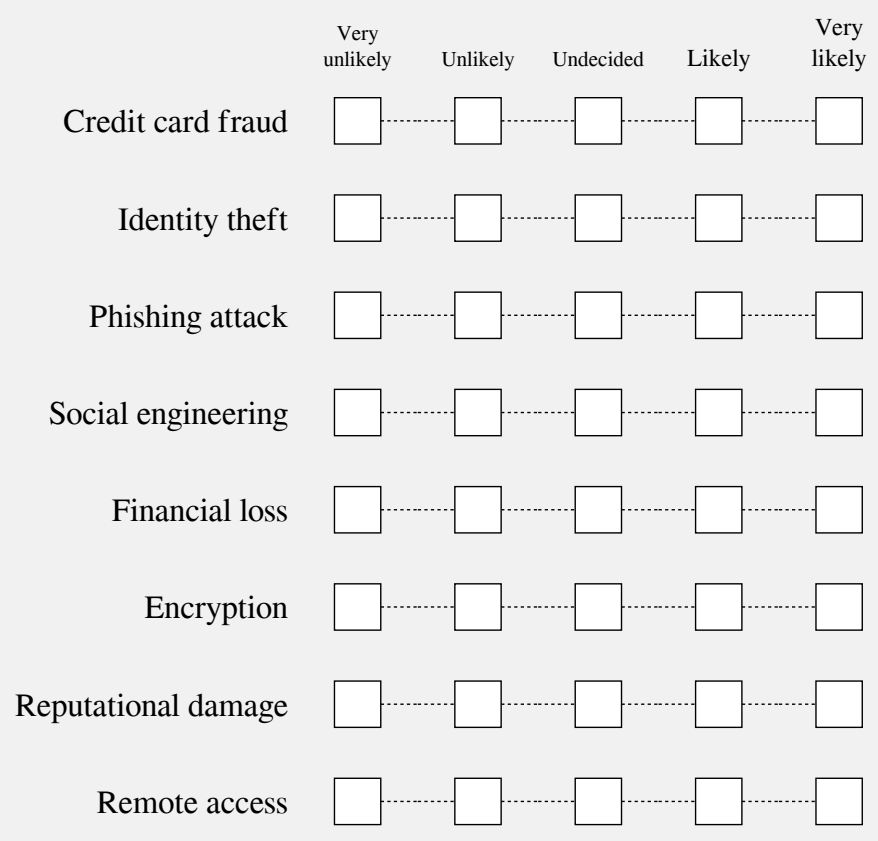

\section{E3. Data breaches happen ...}

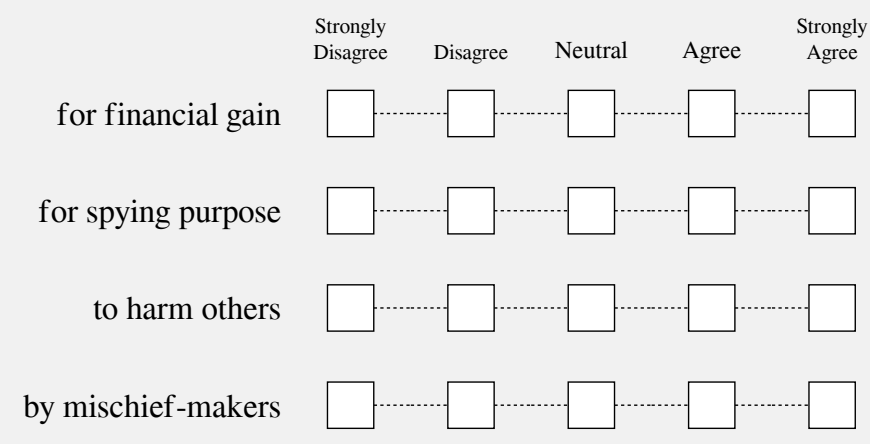

to steal valuable intellectual property 


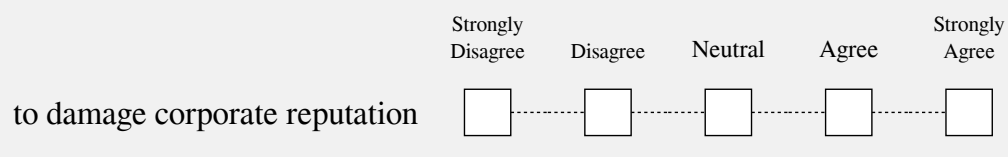

E4. How likely is it to prevent a data breach via the following practices?

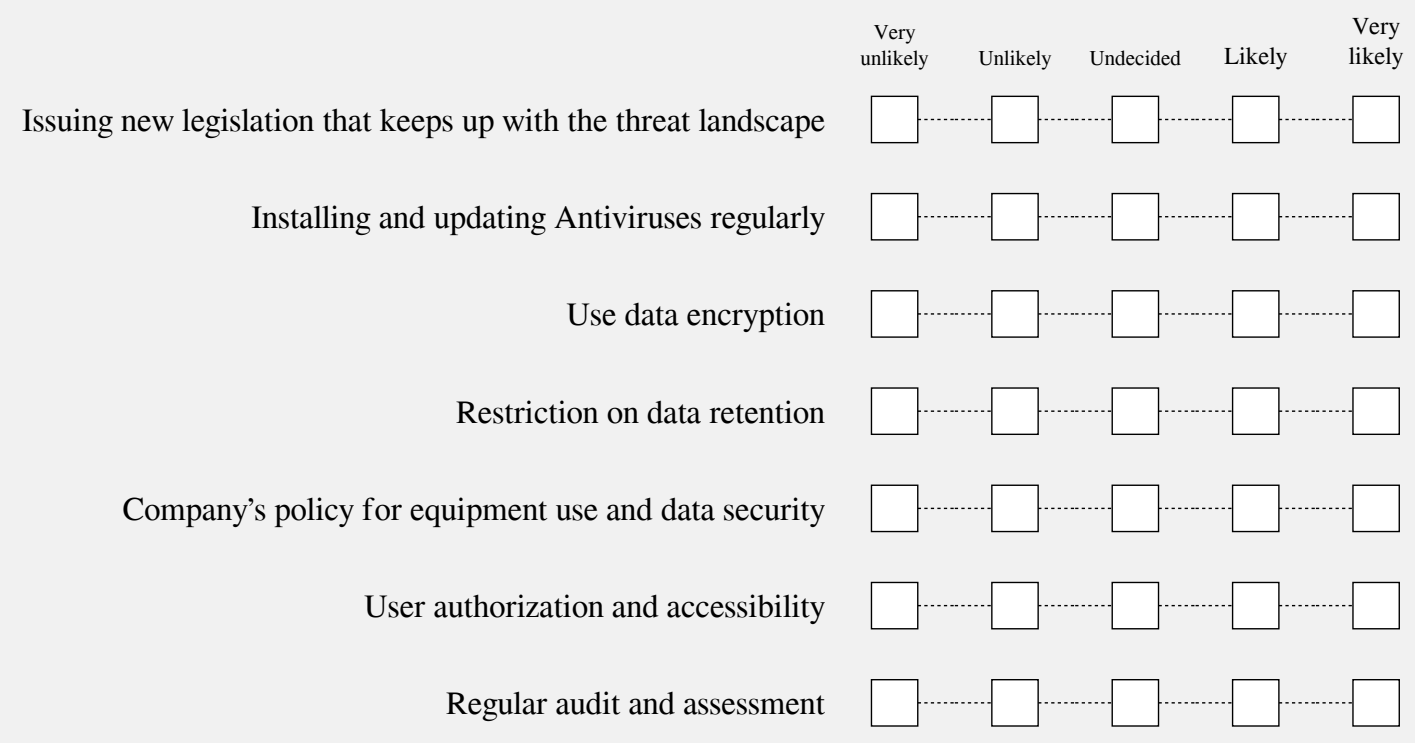

Thank you for taking the survey :) 


\section{Appendix B}

Study 2: Equifax (Nodes, Extract of summary by Code report) 


\begin{tabular}{|c|c|c|c|c|c|c|c|c|}
\hline Nodes & & & & & & & & 2 \\
\hline Name & Files & & References & Created On & Created By & Modified On & Modified By & $\#$ \\
\hline Q. Accountability office & & 0 & 0 & 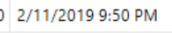 & $\mathrm{ZH}$ & 2/11/2019 9:50 PM & $\mathrm{ZH}$ & \\
\hline helper & & 2 & 11 & 1 2/11/2019 9:51 PM & $\mathrm{ZH}$ & 2/13/2019 5:00 PM & $\mathrm{ZH}$ & \\
\hline object & & 3 & 4 & 4 2/11/2019 9:51 PM & $\mathrm{ZH}$ & 2/13/2019 4:52 PM & $\mathrm{ZH}$ & \\
\hline opponent & & 3 & 16 & 6 2/11/2019 9:51 PM & $\mathrm{ZH}$ & 2/13/2019 5:06 PM & $\mathrm{ZH}$ & \\
\hline reciever & & 0 & 0 & 2/11/2019 9:51 PM & $\mathrm{ZH}$ & 2/11/2019 9:51 PM & $\mathrm{ZH}$ & \\
\hline sender & & 1 & 1 & 2/11/2019 9:51 PM & $\mathrm{ZH}$ & 2/13/2019 4:38 PM & $\mathrm{ZH}$ & \\
\hline Subject & & 2 & 2 & 2 2/11/2019 9:50 PM & $\mathrm{ZH}$ & 2/13/2019 4:50 PM & $\mathrm{ZH}$ & \\
\hline Equifax & & 0 & 0 & $\begin{array}{l}0 \text { 2/3/2019 4:03 PM } \\
\end{array}$ & $\mathrm{ZH}$ & 2/3/2019 4:03 PM & $\mathrm{ZH}$ & \\
\hline helper & & 7 & 93 & 3 2/3/2019 4:04 PM & $\mathrm{ZH}$ & 5/22/2019 2:52 PM & $\mathrm{ZH}$ & \\
\hline object & & 6 & 23 & 3 2/3/2019 4:03 PM & $\mathrm{ZH}$ & 3/22/2019 9:56 AM & $\mathrm{ZH}$ & \\
\hline opponent & & 6 & 27 & 7 2/3/2019 4:04 PM & $\mathrm{ZH}$ & 3/21/2019 5:02 PM & $\mathrm{ZH}$ & \\
\hline reciever & & 7 & 33 & 3 2/3/2019 4:04 PM & $\mathrm{ZH}$ & 3/22/2019 9:57 AM & $\mathrm{ZH}$ & \\
\hline sender & & 3 & 3 & 2/3/2019 4:04 PM & $\mathrm{ZH}$ & 4/17/2019 11:35 AM & $\mathrm{ZH}$ & \\
\hline$\bigcirc$ subject & & 6 & 32 & 2 2/3/2019 4:03 PM & $\mathrm{ZH}$ & 3/22/2019 9:55 AM & $\mathrm{ZH}$ & \\
\hline 10 General news & & 1 & 1 & 1 2/3/2019 4:05 PM & $\mathrm{ZH}$ & 2/15/2019 11:44 AM & $\mathrm{ZH}$ & \\
\hline helper & & 7 & 17 & 7 2/3/2019 4:05 PM & $\mathrm{ZH}$ & 5/22/2019 2:53 PM & $\mathrm{ZH}$ & \\
\hline object & & 8 & 9 & 2/3/2019 4:05 PM & $\mathrm{ZH}$ & 2/17/2019 5:13 PM & $\mathrm{ZH}$ & \\
\hline opponent & & 9 & 32 & 2 2/3/2019 4:06 PM & $\mathrm{ZH}$ & 5/22/2019 2:53 PM & $\mathrm{ZH}$ & \\
\hline reciever & & 5 & 5 & 2/3/2019 4:05 PM & $\mathrm{ZH}$ & 2/17/2019 3:32 PM & $\mathrm{ZH}$ & \\
\hline sender & & 2 & 2 & 2 2/3/2019 4:05 PM & $\mathrm{ZH}$ & 2/17/2019 3:20 PM & $\mathrm{ZH}$ & \\
\hline subject & & 6 & 7 & 7 2/3/2019 4:05 PM & $\mathrm{ZH}$ & 2/17/2019 3:20 PM & $\mathrm{ZH}$ & \\
\hline t. Image repair & & 0 & 0 & 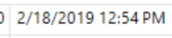 & $\mathrm{ZH}$ & 2/18/2019 12:54 PM & $\mathrm{ZH}$ & \\
\hline$\exists$ Technical & & 0 & 0 & o 2/3/2019 4:06 PM & $\mathrm{ZH}$ & 2/3/2019 4:06 PM & $\mathrm{ZH}$ & \\
\hline$=\bigcirc$ helper & & 0 & 0 & 2/3/2019 4:06 PM & $\mathrm{ZH}$ & $2 / 22 / 201911: 45$ AM & $\mathrm{zH}$ & \\
\hline helper.attacker & & 3 & 12 & 2. 2/22/2019 11:16 AM & $\mathrm{ZH}$ & 2/22/2019 11:44 AM & $\mathrm{ZH}$ & \\
\hline helper. Equifax & & 6 & 6 & 2/22/2019 11:16 AM & $\mathrm{ZH}$ & 2/22/2019 11:45 AM & $\mathrm{ZH}$ & \\
\hline object & & 0 & 0 & 2/3/2019 4:06 PM & $\mathrm{ZH}$ & 2/22/2019 11:07 AM & $\mathrm{ZH}$ & \\
\hline - attacker.object & & 5 & 5 & 2/22/2019 11:05 AM & $\mathrm{ZH}$ & 2/22/2019 11:07 AM & $\mathrm{ZH}$ & \\
\hline Equifax.object & & 1 & 1 & 2/22/2019 11:05 AM & $\mathrm{ZH}$ & 2/22/2019 11:06 AM & $\mathrm{ZH}$ & - \\
\hline
\end{tabular}




\section{Coding Summary By Code Equifax 8/9/2019 1:17 PM}

$\begin{array}{cclll}\text { Aggregate } \quad \text { Classification } & \text { Coverage } & \begin{array}{l}\text { Number } \\ \text { Of Coding } \\ \text { Reference }\end{array} & \begin{array}{l}\text { Reference } \\ \text { Number }\end{array} & \begin{array}{l}\text { Coded By } \\ \text { Initials }\end{array}\end{array}$

\section{Node}

Nodes\\Accountability office\helper

Document

Files\\Accountability Office\|Sep7. 2018

No $0.0731 \quad 3$

$1 \quad \mathrm{ZH} \quad 2 / 13 / 20194: 41 \mathrm{PM}$

We must pass my Data Breach Prevention and Compensation Act to stop these kinds of breaches from happening again."

2

ZH

2/13/2019 4:45 PM

Ranking Member Cummings led all Democrats on the Oversight Committee in requesting that Equifax extend from one year to a minimum of three years the credit protection services the company is currently offering victims of the data breach.

give control of credit information back to consumers.

PDF

Files\\Accountability Office\\Equifax Minority Report - FINAL 12-10-2018

No

$0.0310 \quad 8$

$1 \quad$ ZH $\quad 2 / 13 / 20194: 51 \mathrm{PM}$

via a web-application vulnerability.

2

ZH

2/13/2019 4:51 PM

Equifax failed to implement a patch to fix it-a patch that had been available since March 2017

$3 \quad \mathrm{ZH} \quad 2 / 13 / 20194: 51 \mathrm{PM}$

the credit-reporting giant had more than two months to take precautions that would have defended the personal data of 143 million people from being exposed. 3

ZH

2/13/2019 4:54 PM

hold federal financial regulatory agencies accountable for their consumer protection oversight responsibilities 


$\begin{array}{lcccc}\text { Aggregate Classification } & \text { Coverage } & \begin{array}{l}\text { Number } \\ \text { Of Coding } \\ \text { Reference }\end{array} & \begin{array}{l}\text { Reference } \\ \text { Number }\end{array} & \begin{array}{l}\text { Coded By } \\ \text { Initials }\end{array} \\ & & 5 & \text { ZH }\end{array}$

require federal contractors to comply with established cybersecurity standards and guidance from the National Institute of Standards and Technology (NIST)

$6 \quad$ ZH 2/13/2019 4:54 PM

establish high standards for how data breach victims should be notified

and strengthen the ability of the Federal Trade Commission (FTC) to levy civil penalties for private sector violations of consumer data security requirements

\section{Nodes\\Accountability office|object}

\section{Document}

Files\\Accountability Office\\Sep7. 2018
No
$0.0269 \quad 1$

1

ZH

2/13/2019 4:39 PM

report they requested detailing how attackers exploited significant vulnerabilities at the company to gain access to the sensitive personal information

PDF

Files\\Accountability Office\\2018.09.06 GAO Equifax report

No 0.0002 1

protect sensitive personal information.

Files\\Accountability Office \IEquifax Minority Report - FINAL 12-10-2018

No 0.0014 2

1 ZH 2/13/2019 4:51 PM

Equifax's system 


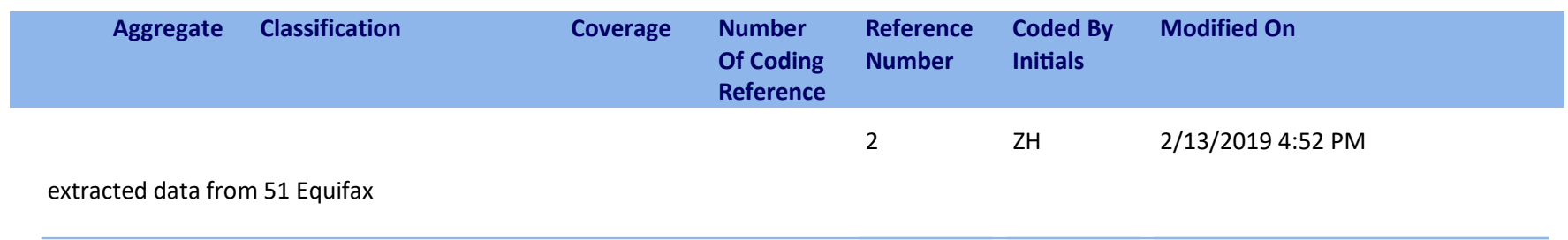

\section{Nodes\\Accountability office\opponent}

\section{Document}

\section{Files\\Accountability Office\|Sep7. 2018}
No
0.1370
5

1

ZH

2/13/2019 4:41 PM

Equifax and other big credit reporting agencies keep profiting off a business model that rewards their failure to protect personal information

$2 \quad$ ZH $\quad 2 / 13 / 20194: 41$ PM

the Trump Administration and Republican-controlled Congress have done nothing.

3

ZH

2/13/2019 4:42 PM

the breakdowns and failures at Equifax that led to one of the largest and most consequential data breaches in United States history

4

$\mathrm{ZH}$

2/13/2019 4:43 PM

The report confirms that the Consumer Financial Protection Bureau (CFPB) and the Federal Trade Commission (FTC) are the key federal regulators responsible for oversight of Credit Ratings Agencies, and both agencies have acknowledged opening investigations after Equifax revealed the breach. But to date, neither investigation has resulted in any enforcement actions against Equifax.

problems with Equifax's response

PDF

Files \Accountability Office\\2018.09.06 Letter to CFPB and FTC re GAO equifax report

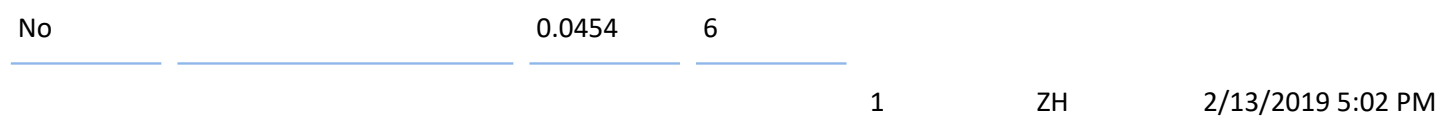

Equifax executives - including its Chief Security Officer and Chief Executive Officer - kept the public in the dark for more than a month after they found out about the security intrusion.

2

ZH

2/13/2019 5:02 PM

No public enforcement actions have been taken by either agency in response to the breach. 


$\begin{array}{lcccc}\text { Aggregate Classification } & \text { Coverage } & \begin{array}{l}\text { Number } \\ \text { Of Coding } \\ \text { Reference }\end{array} & \begin{array}{l}\text { Reference } \\ \text { Number }\end{array} & \begin{array}{l}\text { Coded By } \\ \text { Initials }\end{array} \\ & 3 \text { Modified On } & 2 / 13 / 20195: 04 \text { PM }\end{array}$

These consumer complaints included irnproper use of credit reports, incorrect information on credit reports, inadequate assistance in resolving problems, and problems with Equifax credit 1nonitoring, fraud alerts, and security ffeezes in the wake of the breach. 7

$$
4 \quad \mathrm{ZH} \quad 2 / 13 / 20195: 04 \mathrm{PM}
$$

Eqi1ifax 11ad advance notice of its vul11erabilities and still failed to take steps to protect the personal information of rnillio11s of $A$ n1er1cans.

$5 \quad$ ZH 2/13/2019 5:05 PM

that the breach was caused, in part, by numerous data secttrity failures on the patt of Equifax.

6

ZH

2/13/2019 5:06 PM

yol1r agencies appear to have take11110 definitive action to bold Eqtlifax accountable.

\section{Files\\Accountability Office\\Equifax Minority Report - FINAL 12-10-2018}

No $-\frac{0.0207}{-} \stackrel{5}{1} \quad$ ZH $\quad$ 2/13/2019 4:52 PM

Equifax also failed to provide consumers full protection from new account identity theft.

2

$\mathrm{ZH}$

2/13/2019 4:55 PM

Congress has authorized federal financial regulatory agencies to protect consumers by examining the procedures and controls used by banks and credit reporting agencies, but those powers were not fully exercised before the Equifax data breach.
3
$\mathrm{ZH}$
2/13/2019 4:56 PM

the severe deficiencies in Equifax's security patch implementation

4

$\mathrm{ZH}$

2/13/2019 4:57 PM

These existing authorities were not used effectively with respect to Equifax.

\section{5}

In the three years before the Equifax data breach, the company spent only about $3 \%$ of its operating revenue on cybersecurity-less than the company spent on stock dividends. 


$\begin{array}{lllll}\text { Aggregate } & \text { Classification Coverage } & \begin{array}{l}\text { Number } \\ \text { Of Coding } \\ \text { Reference }\end{array} & \begin{array}{l}\text { Reference } \\ \text { Number }\end{array} & \begin{array}{l}\text { Coded By } \\ \text { Initials }\end{array}\end{array}$

Nodes\\Accountability office\sender

Document

Files\\Accountability Office\|Sep7. 2018

No $0.0231 \quad 1$

1

$\mathrm{ZH}$

2/13/2019 4:38 PM

Warren (D-Mass.) and Rep. Elijah E. Cummings (D-Md.), the Ranking Member of the House Committee on Oversight and Government Reform

Nodes\\Accountability office\Subject

Document

Files\\Accountability Office\|Sep7. 2018

$\begin{array}{lll}\text { No } & 0.0066 & 1\end{array}$

1

ZH

2/13/2019 4:39 PM

Government Accountability Office (GAO

PDF

Files \|Accountability Office\\Equifax Minority Report - FINAL 12-10-2018

$\begin{array}{lll}\text { No } & 0.0002 & 1\end{array}$

1

$\mathrm{ZH}$

2/13/2019 4:50 PM

attackers 


$\begin{array}{cclll}\text { Aggregate } \quad \text { Classification } & \text { Coverage } & \begin{array}{l}\text { Number } \\ \text { Of Coding } \\ \text { Reference }\end{array} & \begin{array}{l}\text { Reference } \\ \text { Number }\end{array} & \begin{array}{l}\text { Coded By } \\ \text { Initials }\end{array}\end{array}$

Nodes\\Equifax\helper

PDF

\section{Files\\Equifax press release\\01-31-2018}
No
0.1224
6
1
ZH
3/21/2019 3:20 PM

U.S. consumers who are 18 years or older and have an Equifax credit report may enroll in this service, which is available for free, for life.

2

$\mathrm{ZH}$

3/21/2019 3:20 PM

Once a consumer has enrolled in the service, Equifax will send an alert each time he or she locks or unlocks their Equifax credit report.

3

ZH

3/21/2019 3:21 PM

to provide a service that allows consumers to help control access to their personal Equifax credit report in an easy and convenient way.

$4 \quad$ ZH $\quad 3 / 21 / 20193: 21 \mathrm{PM}$

Not only does Lock \& Alert empower consumers to help restrict certain access to their Equifax credit report, which may help better protect against unauthorized access and identity theft, it also gives them the ability to lock and unlock their report through a convenient app on their mobile device,

$5 \quad$ ZH $\quad 3 / 21 / 20193: 22 \mathrm{PM}$

It also has no associated fees.

"The consumer-empowerment approach that is offered through Lock \& Alert is what people have come to expect,"

Files\\Equifax press release\\03-01-2018-140531340

$\stackrel{\text { No }}{-} \longrightarrow$

$1 \quad$ ZH $\quad 3 / 21 / 20193: 25$ PM

ongoing analysis of data stolen

2

ZH $\quad 3 / 21 / 20193: 29$ PM

Equifax was able to identify these consumers by referencing other information in proprietary company records that the attackers did not steal, and by engaging the resources of an external data provider.

$3 \quad \mathrm{ZH} \quad 3 / 21 / 20193: 29 \mathrm{PM}$

Through these additional efforts, Equifax was able to identify approximately 2.4 million U.S. consumers whose names and partial driver's license information were stolen,

$4 \quad \mathrm{ZH} \quad 3 / 21 / 20193: 30 \mathrm{PM}$

This information was partial because, in the vast majority of cases, it did not include consumers' home addresses, or their respective driver's license states, dates of issuance, or expiration dates.

$$
5 \quad \mathrm{ZH} \quad 3 / 21 / 20193: 41 \mathrm{PM}
$$

The methodology used in the company's forensic examination of last year's cybersecurity incident leveraged Social Security Numbers (SSNs) and names as the key data elements to identify who was affected by the cyberattack. 

Aggregate Classification
Coverage
Number
Reference
Coded By
Modified On
Of Coding Number
Initials

Reference

$\mathrm{ZH}$

3/21/2019 3:48 PM

newly discovered stolen data,

$\mathrm{ZH}$

3/21/2019 3:48 PM

analyzing other information in our databases that was not taken by the attackers, and making connections that enabled us to identify additional individuals."
8
$\mathrm{ZH}$
3/21/2019 3:49 PM

Equifax will notify these newly identified U.S. consumers directly

$9 \quad \mathrm{ZH} \quad 3 / 21 / 20193: 49$ PM

will offer identity theft protection and credit file monitoring services at no cost to them

\section{0}

$\mathrm{ZH}$

3/21/2019 3:49 PM

Information about registering for these services will be included in the notification.

$11 \quad \mathrm{ZH} \quad 3 / 21 / 20193: 50 \mathrm{PM}$

"We continue to take broad measures to identify, inform, and protect consumers who may have been affected by this cyberattack,"

$12 \quad \mathrm{ZH} \quad 3 / 21 / 20193: 50 \mathrm{PM}$

We are committed to regaining the trust of consumers

$13 \quad$ ZH $\quad 3 / 21 / 20193: 50 \mathrm{PM}$

improving transparency

$14 \quad \mathrm{ZH} \quad 3 / 21 / 20193: 51$ PM

and enhancing security across our network."

$15 \quad \mathrm{ZH} \quad 3 / 21 / 20193: 58 \mathrm{PM}$

Equifax launched Lock \& Alert ${ }^{\mathrm{TM}}$ to all U.S. consumers on January 31.

$16 \quad \mathrm{ZH} \quad 3 / 21 / 20193: 58$ PM

This new service, which is free for life, enables consumers to quickly lock and unlock their Equifax credit report using a computer or app downloaded on their mobile device.

$17 \quad \mathrm{ZH} \quad 3 / 21 / 20193: 59 \mathrm{PM}$

have found no evidence that Equifax's core consumer, employment and income, or commercial credit reporting databases were accessed as part of the cyberattack, and the company believes it will have met all applicable requirements to notify consumers.
18
$\mathrm{ZH}$
3/21/2019 4:00 PM

Since announcing this incident, Equifax has taken steps to communicate with and assist consumers and customers.

$19 \quad \mathrm{ZH} \quad 3 / 21 / 20194: 01 \mathrm{PM}$

Among other things, the company has established a web portal advising U.S. consumers to review their account statements and credit reports

identify any unauthorized activity, 

Aggregate Classification
Coverage
Number
Coded By Modified On
Of Coding Number
Initials
Reference
$21 \quad$ ZH $\quad 3 / 21 / 20194: 01$ PM

and protect their personal information from further attack.

$22 \quad \mathrm{ZH} \quad 3 / 21 / 20194: 01 \mathrm{PM}$

Additionally, the company offered free identity theft protection and credit file monitoring services to all U.S. consumers regardless of whether or not they were impacted.

$23 \quad \mathrm{ZH} \quad 3 / 21 / 20194: 02 \mathrm{PM}$

Consumers can visit www.equifaxsecurity2017.com for more information about the cybersecurity incident and for answers to frequently asked questions.

$24 \quad$ ZH $\quad 3 / 21 / 20194: 03$ PM

including additional analysis of the data stolen

$25 \quad$ ZH $\quad 3 / 21 / 20194: 03$ PM

the government investigations and litigation resulting from the 2017 cybersecurity incident

$26 \quad \mathrm{ZH} \quad 3 / 21 / 20194: 04 \mathrm{PM}$

Equifax is a global information solutions company that uses unique data, innovative analytics, technology and industry expertise to power organizations and individuals

$27 \quad \mathrm{ZH} \quad 3 / 21 / 20194: 05$ PM

by transforming knowledge into insights that help make more informed business and personal decisions.

Files\\Equifax press release $\ \mid 05-07-2018$

No $\quad 0.1284 \quad 4$

$1 \quad \mathrm{ZH} \quad 3 / 21 / 20194: 13 \mathrm{PM}$

which Equifax is disclosing publicly as part of its commitment to transparency,

ZH $\quad 3 / 21 / 20194: 14$ PM

the Company has now provided the approximate number of impacted U.S. consumers for each of those data elements.

$3 \quad \mathrm{ZH} \quad 3 / 21 / 20194: 15 \mathrm{PM}$

the Company has provided details on the images accessed by the attackers from Equifax's dispute portal, the theft of which had been disclosed in the Company's September 2017 press release.

$4 \quad$ ZH $\quad 3 / 21 / 20194: 16$ PM

Equifax is confident that the additional detail about the 2017 cybersecurity incident does not identify new stolen data or newly impacted consumers and does not require additional consumer notification.

\section{Files\|Equifax press release\|09-07-2017-213000628}

No 0.0907

11

No Evidence of Unauthorized Access to Core Consumer or Commercial Credit Reporting Databases 


\section{Appendix C}

Study 2: Marriott Hotels (Extract of summary by Code report) 


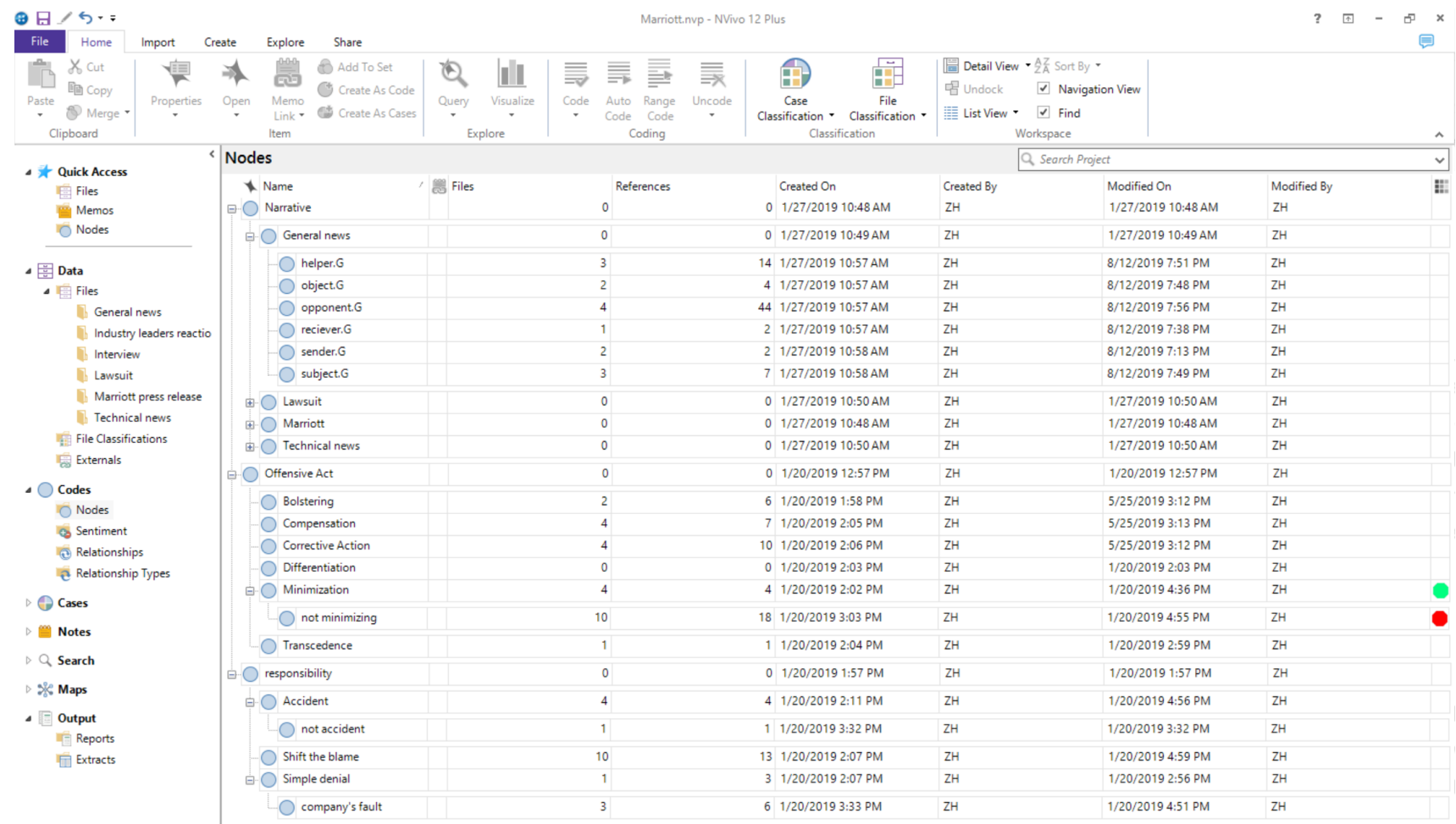




\section{Coding Summary By Code thesis 8/9/2019 1:54 PM}

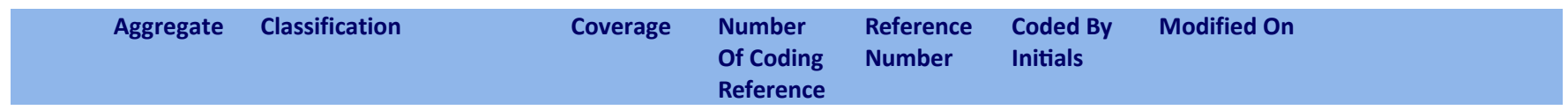

\section{Node}

Nodes\|Narrative\General news\helper.G

\section{Document}

Files\|General news\\NYT, 4 Jan 2019

No $0.0586 \quad 3$

$1 \quad$ ZH $\quad 1 / 30 / 201912: 13$ PM

An additional 20.3 million passport numbers were kept in encrypted files, which would require a master encryption key to read.

$2 \quad \mathrm{ZH} \quad 1 / 30 / 2019$ 12:15 PM

Marriott has said it would pay for a new passport for anyone whose passport information, hacked from their systems, was found to be involved in a fraud.

3

$\mathrm{ZH}$

1/30/2019 12:16 PM

The company also said that about 8.6 million credit and debit cards were "involved" in the incident, but those are all encrypted -

Nodes\\Narrative\General news\object.G

Document

Files\\General news\\The Washington Post. 4 Jan 2019

No $0.0033 \quad 1$

1 

Aggregate Classification
Coverage
Number
Reference
Coded By
Modified On
Of Coding Number Initials
Reference

Nodes\\Narrative\General news\opponent.G

\section{Document}

Files\|General news\|NYT, 4 Jan 2019
No
0.1278
6
1
$\mathrm{ZH}$
1/30/2019 12:10 PM

its Starwood hotel unit did not encrypt the passport numbers for roughly five million guests.

$\mathrm{ZH}$

1/30/2019 12:10 PM

an attack that many outside experts believe was carried out by Chinese intelligence agencies.

3

$\mathrm{ZH}$

1/30/2019 12:11 PM

the presence of passport numbers, which could make it far easier for an intelligence service to track people who cross borders.

$4 \quad \mathrm{ZH} \quad 1 / 30 / 201912: 12 \mathrm{PM}$

the attack appeared to be part of a broader effort by China's Ministry of State Security to compile a huge database of Americans and others with sensitive government or industry positions

$5 \quad \mathrm{ZH} \quad 1 / 30 / 201912: 13 \mathrm{PM}$

Marriott said for the first time that 5.25 million passport numbers were kept in the Starwood system in plain, unencrypted data files meaning they were easily read by anyone inside the reservation system.

$6 \quad \mathrm{ZH} \quad 1 / 30 / 201912: 15 \mathrm{PM}$

But that was something of a corporate sleight of hand, since it provided no coverage for guests who wanted a new passport simply because their data had been taken by foreign spies.

\section{Files\\General news\\The Washington Post. 4 Jan 2019}

No $-\frac{0.1071}{-} \stackrel{3}{1} \quad$ ZH $\quad 1 / 30 / 2019$ 12:05 PM

the hackers were working on behalf of the Chinese Ministry of State Security

$2 \quad \mathrm{ZH} \quad 1 / 30 / 201912: 05 \mathrm{PM}$

The hackers accessed about 20.3 million encrypted passport numbers.

$3 \quad \mathrm{ZH} \quad 1 / 30 / 201912: 06 \mathrm{PM}$

Unencrypted passport numbers are valuable to state intelligence agencies because they can be used to compile detailed dossiers on people and their international movements. 


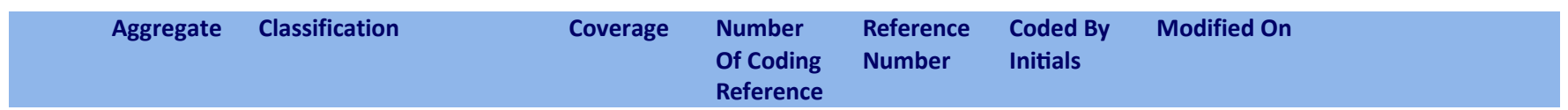

Nodes\\Narrative\General news\sender.G

Document

Files \|General news \|The Washington Post. 4 Jan 2019

$\begin{array}{lll}\text { No } & 0.0183 & 1\end{array}$

1

$\mathrm{ZH}$

1/30/2019 12:04 PM

The FBI is leading the investigation of the data theft

Nodes\\Narrative\General news\subject.G

Document

Files \|General news \|The Washington Post. 4 Jan 2019

No $0.0444 \quad 1$

$1 \quad$ ZH $\quad 1 / 30 / 2019$ 12:04 PM

investigators suspect the hackers were working on behalf of the Chinese Ministry of State Security, the rough equivalent of the CIA

Nodes\\Narrative\Lawsuit|object.L

PDF

Files\\Lawsuit\|2019-01-15-class-action-complaint-marriott-starwood

No $-\frac{0.0001}{2} \frac{1}{1} \quad$ ZH $\quad 1 / 27 / 2019$ 11:24 AM

data protection 


$\begin{array}{cclll}\text { Aggregate } \quad \text { Classification } & \text { Coverage } & \begin{array}{l}\text { Number } \\ \text { Of Coding } \\ \text { Reference }\end{array} & \begin{array}{l}\text { Reference } \\ \text { Number }\end{array} & \begin{array}{l}\text { Coded By } \\ \text { Initials }\end{array}\end{array}$

Nodes\\Narrative\Lawsuit\Opponent.L

PDF

\section{Files\\Lawsuit\|2019-01-15-class-action-complaint-marriott-starwood}

No 0.0385

26

1

$\mathrm{ZH}$

1/27/2019 11:09 AM

the company must be at the forefront of data security to ensure that thieves and hackers could never get access to the data the company has collected.

$2 \quad \mathrm{ZH} \quad 1 / 27 / 201911: 10 \mathrm{AM}$

It cannot let its security lie fallow for years

$3 \quad \mathrm{ZH} \quad 1 / 27 / 201911: 10 \mathrm{AM}$

failing to update to the latest security

$4 \quad \mathrm{ZH} \quad 1 / 27 / 2019$ 11:10 AM

failing to patch critical software effectively and promptly

$5 \quad$ ZH $\quad 1 / 27 / 201911: 11$ AM

failing to invest in data security at sufficient levels,

$6 \quad \mathrm{ZH} \quad$ 1/27/2019 11:11 AM

and especially failing to detect gaping holes in security that have been present and hemorrhaging data for years on end.

$7 \quad \mathrm{ZH} \quad 1 / 27 / 201911: 11 \mathrm{AM}$

a hospitality company must immediately and accurately notify all those affected to prevent consumers from becoming victims of identity theft.

$8 \quad \mathrm{ZH} \quad 1 / 27 / 201911: 12 \mathrm{AM}$

And it must take immediate steps to mitigate the damages it has caused. This lawsuit stems from Defendants abject failure to follow these simple rules.

$9 \quad \mathrm{ZH} \quad 1 / 27 / 201911: 17 \mathrm{AM}$

The data stolen is extremely valuable to identity thieves because it contains elements that can be used to falsely verify fraudulent accounts, like passport numbers, specific travel dates, gender, dates of birth, email addresses, phone numbers, and for millions of victims, payment card information

$10 \quad \mathrm{ZH} \quad 1 / 27 / 201911: 18 \mathrm{AM}$

While Defendants claim to have encrypted payment card information, they took no similar steps to protect the even more valuable personal information like passport numbers and travel details that they collected

$11 \quad \mathrm{ZH} \quad 1 / 27 / 201911: 18 \mathrm{AM}$

Defendants plainly were asleep at the wheel as this data was being exfiltrated from their systems for at least four years while they had no idea the breach was ongoing.

$12 \quad \mathrm{ZH} \quad 1 / 27 / 201911: 18 \mathrm{AM}$

Marriott negligently left its Starwood computer systems vulnerable to the breach.

$13 \quad \mathrm{ZH} \quad 1 / 27 / 201911: 20 \mathrm{AM}$

The massive data breach could have been prevented and should have been detected and disclosed earlier. 

Aggregate Classification
Coverage
Number
Reference
Coded By
Modified On
Of Coding
Number
Initials
Reference
14
ZH
1/27/2019 11:21 AM

Marriott knew that hackers valued

the data it and Starwood stored as it was often targeted, and often its systems were compromised yet data security was still neglected and under-supported by Defendants

$15 \quad \mathrm{ZH} \quad 1 / 27 / 201911: 23$ AM

As recently as November 2015,

$16 \quad \mathrm{ZH} \quad 1 / 27 / 2019$ 11:23 AM

systems at 54 of its hotels in North America had been attacked, were infected with malware, and customer payment data was being stolen by hackers.

$17 \quad \mathrm{ZH} \quad 1 / 27 / 2019$ 11:25 AM

clearly Marriott and Starwood did not and do not take

data protection seriously enough to protect their customers information from hackers, or to even know that for four long years, hackers have been stealing their customers sensitive data at will and without detection.

$18 \quad \mathrm{ZH} \quad 1 / 27 / 201911: 26$ AM

Instead of promptly detecting and promptly notifying the hundreds of millions of consumers whose personal information was stolen, Marriott said nothing, leaving $r$ at least three months between the time the breach was first detected and the time Marriott publically announced it.

$19 \quad \mathrm{ZH} \quad 1 / 27 / 2019$ 11:27 AM

Marriott is acutely aware that the consumer and business information it stores is highly sensitive and highly valuable to identity thieves and other criminals.

$20 \quad \mathrm{ZH} \quad 1 / 27 / 201911: 28 \mathrm{AM}$

On this same web page, Marriott makes it clear that customers do not have the option not to provide it with their personal information.

$21 \quad \mathrm{ZH} \quad 1 / 27 / 201911: 28 \mathrm{AM}$

Marriott brazenly puts the impetus on its customers to themselves detect if their information is being stolen and then report it to Marriott.

$22 \quad \mathrm{ZH} \quad 1 / 27 / 201911: 29 \mathrm{AM}$

While Marriott says it encrypted payment card data (though it also admits the keys to decrypt it may have also been stolen), Marriott should also have converted nformation into coded strings that would not be immediately useful, or even identifiable to cyber-thieves.

$23 \mathrm{ZH} \quad 1 / 27 / 201911: 30 \mathrm{AM}$

Immediate notice of a data breach is essential to obtain the best protection

afforded by identity theft protection services. Marriott failed to provide such immediate notice, thus further exacerbating the damages sustained by Plaintiff and the Nationwide Class resulting from the breach.

$24 \quad \mathrm{ZH} \quad 1 / 27 / 201911: 31 \mathrm{AM}$

Marriott knew its systems were compromised at least as early as September 8, 2018, yet it made no disclosures until November $30,2018$.

$25 \quad \mathrm{ZH} \quad 1 / 27 / 201911: 31 \mathrm{AM}$

When Marriott finally did act, it set up a separately hosted website to provide customers information and sent emails from easily spoofed accounts, failing to register for itself domains that cyber-thieves could use to further defraud Marriott customers. 


\section{Appendix D}

Study 3: Consent Form, Questionnaire 


\title{
Section A: Research Consent Form
}

\section{Name and Contact Information of Researchers:}

\author{
Zahra Hassanzadeh, Carleton University, School of Computer Science
}

Email: zahrahassanzadeh@cmail.carleton.ca

Supervisor and Contact Information:

Dr. Robert Biddle, Carleton University, School of Computer Science

Email: Robert.Biddle@carleton.ca

\section{Project Title}

Exploring Mental Models of Internet data breaches

\section{Project Sponsor and Funder (if any)}

NSERC Discovery Grant RGPIN-2016-06149 "New Directions in Usable Security"

\section{Carleton University Project Clearance}

CUREB-B Clearance \#: 110780

\section{What is the purpose of the study?}

In this project, we will conduct a user study to explore users' mental model of Internet data breaches. A data breach happens when an unauthorised person accesses private or confidential information.

\section{What will I be asked to do?}

If you agree to take part in the study, we will ask you to fill out one demographic questionnaire, then you will be asked to answer some questions about causes, consequences, motivation and prevention methods of a data breach. Afterwards, we will ask you to read two paragraphs and answer some questions. This study may take approximately 15 minutes.

\section{Risks and Inconveniences}

We do not anticipate any risks to participating in this study.

\section{Compensation/Incentives}

You will be paid $\$ 2$ for your participation in this study.

\section{No waiver of your rights}

By signing this form, you are not waiving any rights or releasing the researchers from any liability. 


\section{Withdrawing from the study}

You may decline to answer any question and you may withdraw from the study at any point without providing justification. Withdrawal is only possible until the survey is submitted, since the online survey will be anonymous. If you withdraw from the study, all information you have provided will be immediately destroyed.

\section{Confidentiality}

We will treat your personal information as confidential. None of your personal information will be accessed, nor will you be asked to disclose personally identifiable information. Research records may be accessed by the Carleton University Research Ethics Board in order to ensure continuing ethics compliance.

The results of this study will be used for a Master thesis and it may be published or presented at an academic conference or meeting later but the data will be presented so that it will not be possible to identify any participants. There are no potential risks to you as your personal data will not be required. Your identity will be protected for any information you do choose to provide. This will be done by keeping all responses anonymous and allowing you to request that certain responses not be included in the final thesis.

Our questionnaires are hosted on Limesurvey installed on our research lab's server. This server is secure: it has daily anti-malware checks, is password-protected, and is located in a secure server room at 2118 HCI Building. Your data will be stored and protected by Carleton University's HotSoft research lab in the HCI Building on the Carleton campus but may be disclosed via a court order or data breach. All data, including coded information, will be kept in a password-protected computer. Only researchers directly involved in the research will have access to the study data. This data may be used for future research on a related topic.

\section{Ethics review}

This project was reviewed and cleared by the Carleton University Research Ethics Board. If you have any ethical concerns with the study, please contact Dr. Bernadette Campbell, Chair, Carleton University Research Ethics Board-B (by phone at 613-520-2600 ext. 4085 or by email at ethics@ carleton.ca). For all other questions about the study, please contact the researcher.

\section{Statement of consent}

I voluntarily agree to participate in this study.

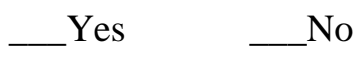




\section{Section B: Demographics Questionnaire}

Participants Demographic Survey

B1. What is your age?

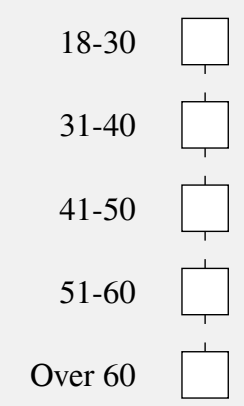

B2. What is your gender?

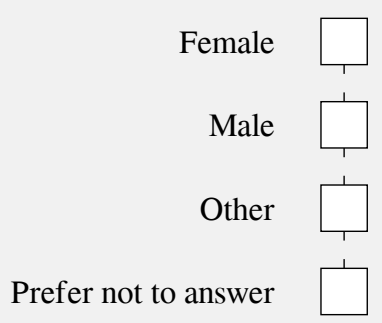

B3. What is your highest level of education?

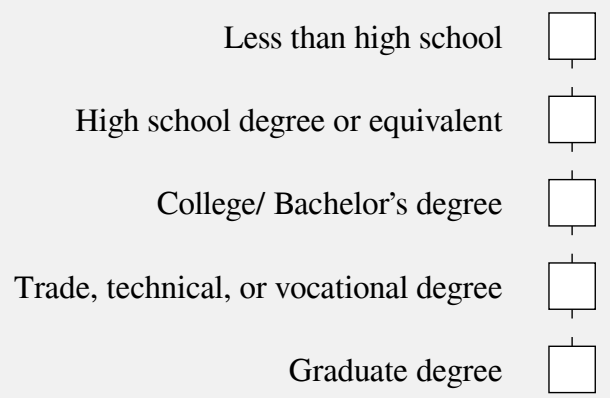

B4. What is/was your major field of study?

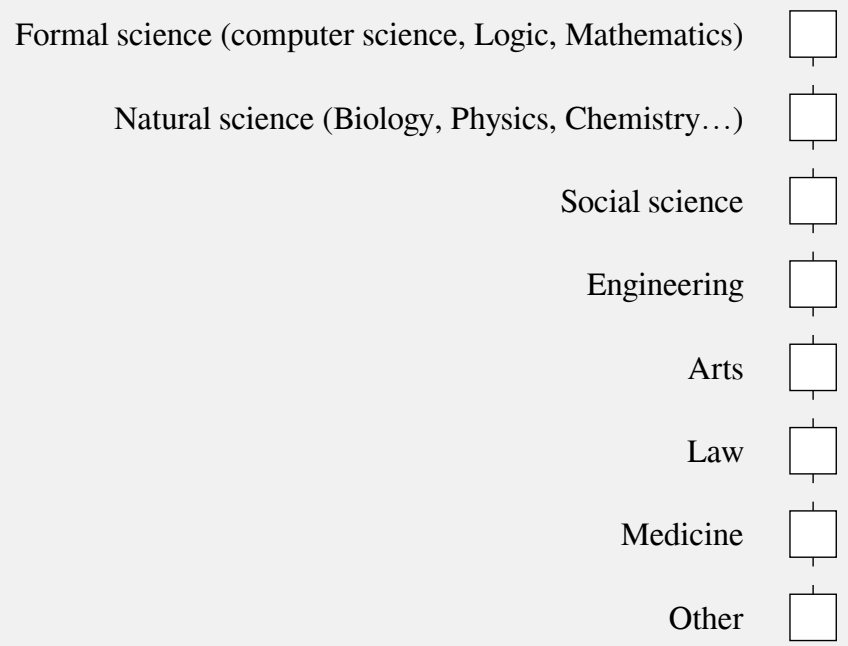


B5. Which country do you live?

Canada

US

Other

\section{Section C: Security Experience}

\section{C1. Indicate the extent to which you agree or disagree with the following statements:}

I have experienced a data security problem, like someone trying to secretly change my password.

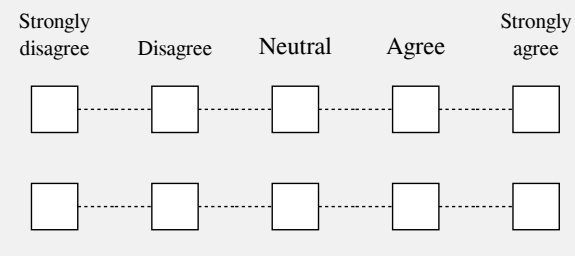

I have experienced identity theft, like someone applying for credit card using my information.

\section{C2. Indicate the extent to which you agree or disagree with the following statements:}

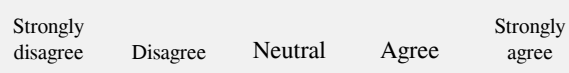

A vulnerability is a weakness which can be exploited by an attacker to perform unauthorized actions within a computer system.

A backdoor is a means to access to a computer system or encrypted data that bypasses the system's customary security mechanisms.

There are security expert teams around the world that provide timely information about current security issues, vulnerabilities, and exploits.

The public announcement of security issues and vulnerabilities can cause a data breach.

It is helpful for companies to have a reference lists of publicly known information-security vulnerabilities and exposures.

It is an opportunity for attackers to have access to a reference lists of publicly known information-security vulnerabilities and exposures.

In some countries, there are regulations on data protection and privacy for all individuals.
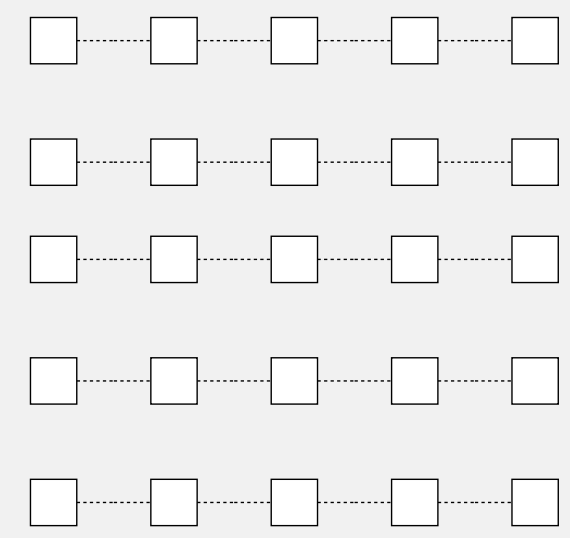

\section{Section D: SeBIS}

Security Behavior Intentions Scales

\section{D1. Security Behavior Intentions Scales}

I set my computer screen to automatically lock if I don't use it for a prolonged period of time.

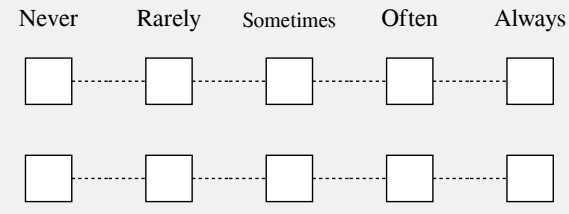

I use a password/passcode to unlock my laptop or tablet. 
I manually lock my computer screen when I step away from it.

I use a PIN or passcode to unlock my mobile phone.

I do not change my passwords, unless I have to.

I use different passwords for different accounts that I have.

When I create a new online account, I try to use a password that goes beyond the site's minimum requirements.

I do not include special characters in my password if it's not required.

When someone sends me a link, I open it without first verifying where it goes.

I know what website I'm visiting based on its look and feel, rather than by looking at the URL bar.

I submit information to websites without first verifying that it will be sent securely (e.g., SSL, "https://", a lock icon).

When browsing websites, I mouseover links to see where they go, before clicking them.

If I discover a security problem, I continue what I was doing because I assume someone else will fix it.

When I'm prompted about a software update, I install it right away.

I try to make sure that the programs I use are up-to-date.

I verify that my anti-virus software has been regularly updating itself.

Never Rarely Sometimes Often Always
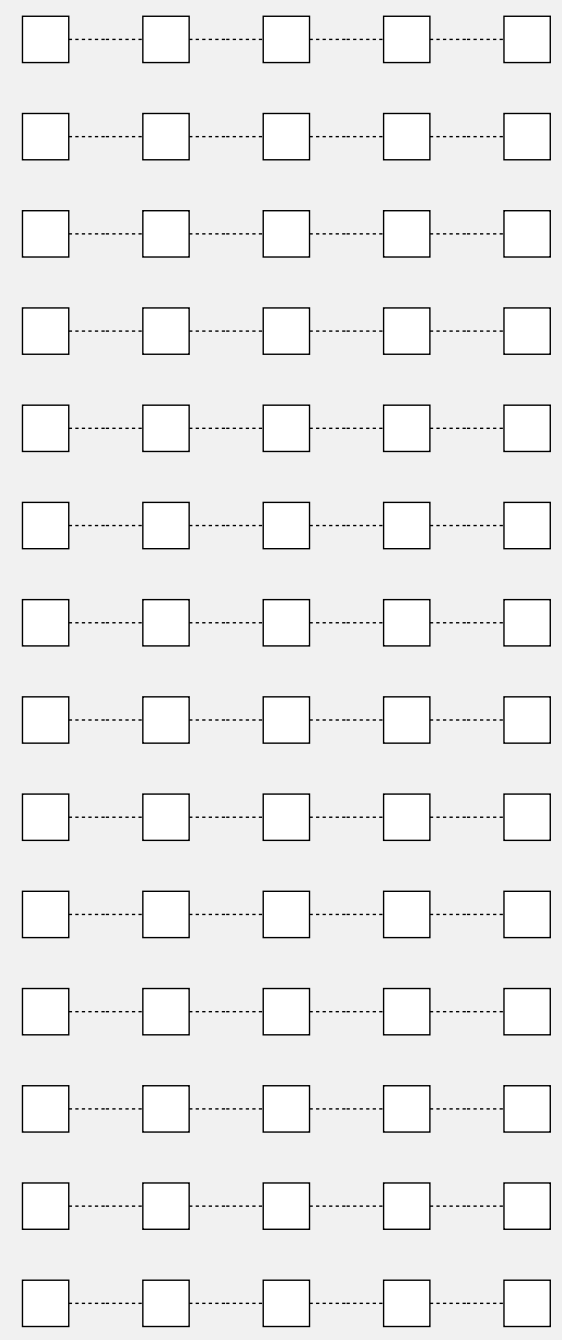

\section{Section E: Data Breach Questions}

\section{E1. Which of the following are causes of data breach?}

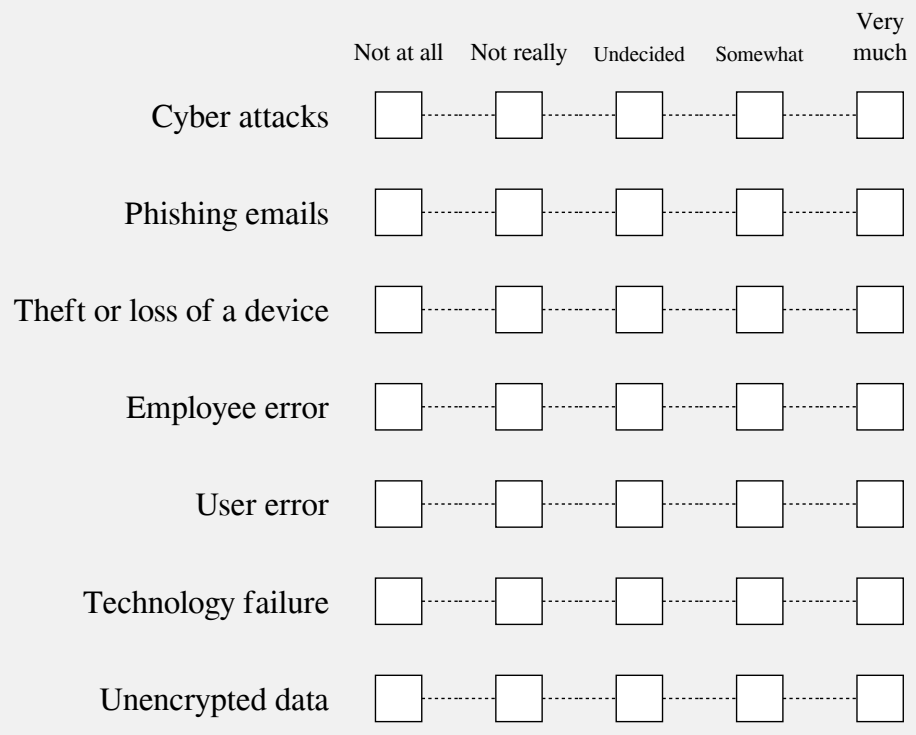




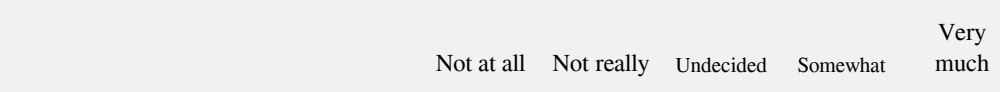

Peer-to-peer file transfer

Lack of data access monitoring

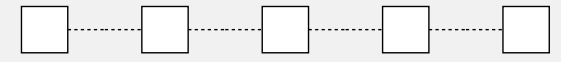

Not updating software

Using tactics to fool people to disclose their personal information

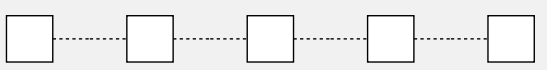

Malware (e.g. virus)

System security vulnerabilities
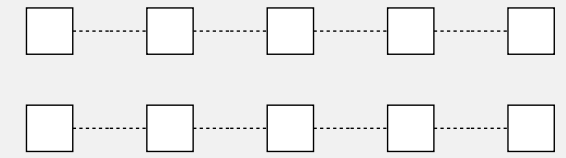

E2. How likely is that the following items happen as a consequence of an Internet data breach?

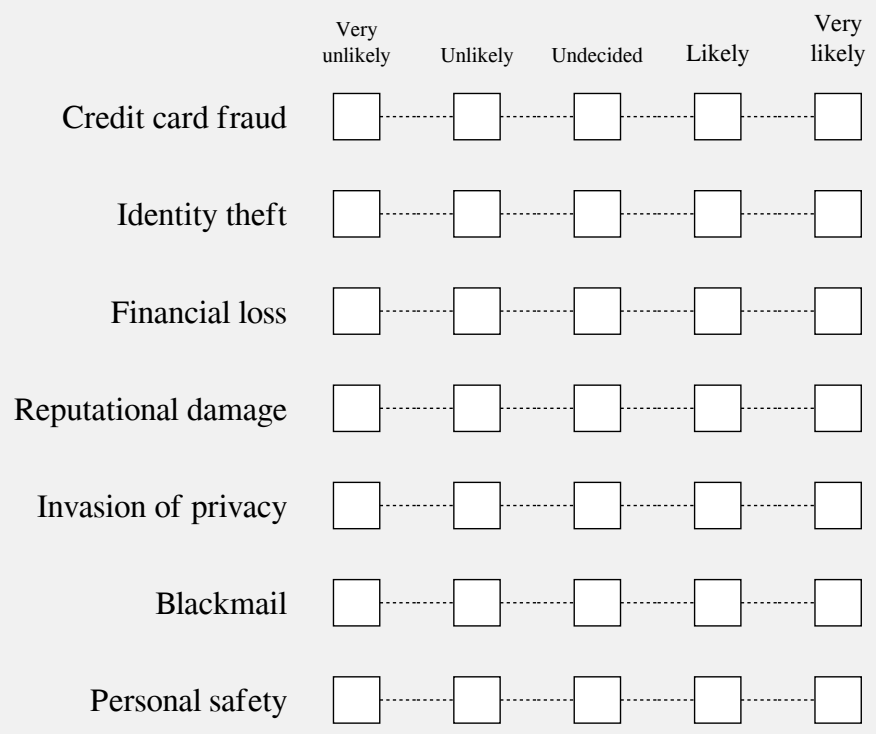

\section{E3. Data breaches happen ...}

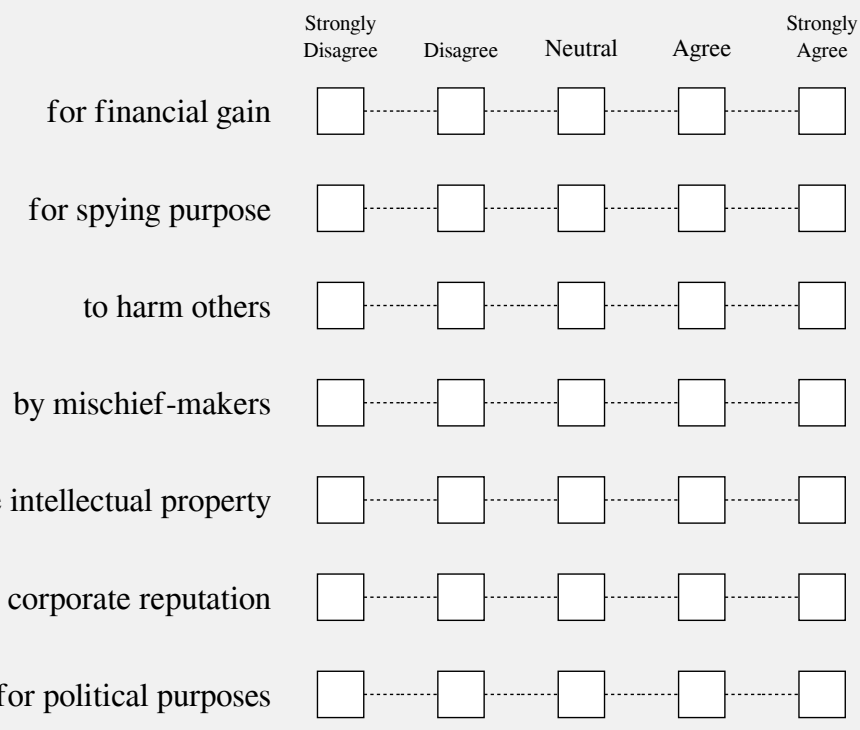


E4. How likely would the following practices prevent a data breach?

\section{||| ||| ||||||||||||||||||||}

Issuing new legislation that keeps up with new threat

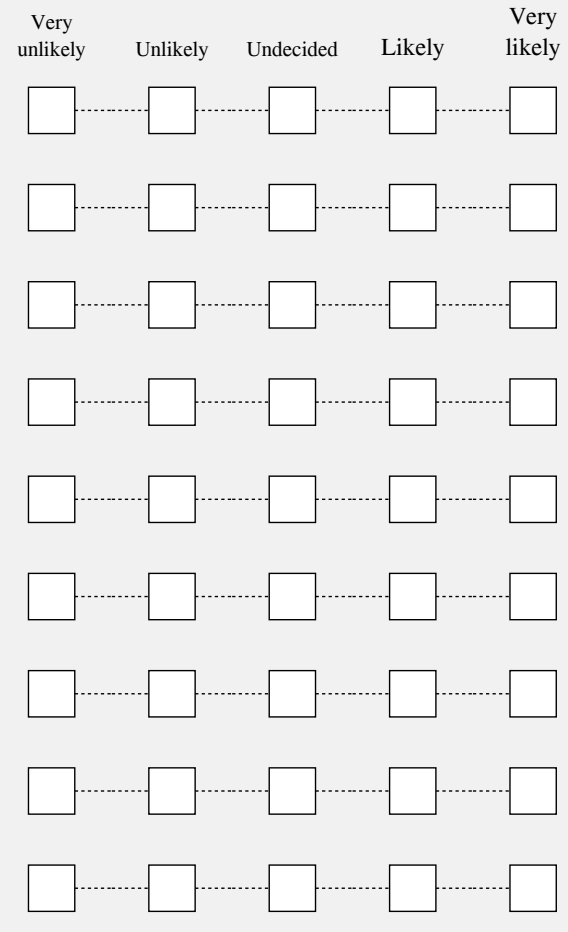

Keeping all the software and hardware updated

Fixing or improving all the software

Using data encryption

Restriction on data retention (e.g. time limits)

More strict policy for equipment use and data security

Restricting user authorization and accessibility

Regular audit and assessment

Using intrusion detection systems

Using multi-factor authentication (e.g. Password and fingerprints)

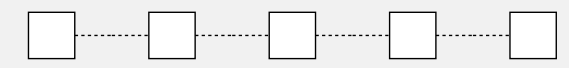

E5. How would you rate the accountability of the following people for data protection in an enterprise data breach?

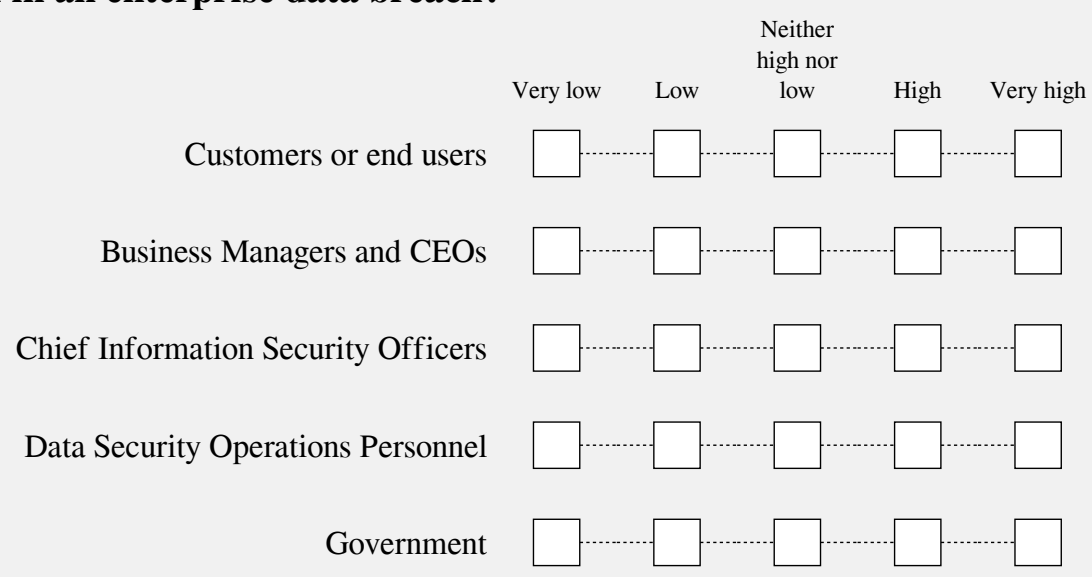

Expert groups that identify security vulnerabilities

E6. $\mathbf{Q}$ 


\section{Section F: Data Breach Description}

F1. Read the following extract carefully and indicate the extent to which you agree or disagree with the following statements. Company A Data Breach Statement:

"Throughout my tenure as CEO of the company, we took data security and privacy extremely seriously, and we devoted substantial resources to it. The company failed to prevent sensitive information from falling into the hands of wrongdoers. The people affected by this are not numbers in a database. They are my friends, my family, members of my church, the members of my community, my neighbors. This breach has impacted all of them. It has impacted all of us... We now know that criminals executed a major cyberattack, hacked into our data, and were able to access information for over 140 million American consumers... Upon learning of suspicious activity, I and many others at the company worked with outside experts to understand what had occurred and do everything possible to make this right. Ultimately, we realized we had been the victim of a massive theft, and we set out to notify American consumers, protect against increased attacks, and remediate and protect against harm to consumers. We developed a robust package of remedial protections for each and every American consumer - not just those affected by the breach - to protect their credit information."

The customers of the company are victims of the data breach.

The company is the victim of the data breach.
The company had a relaxed attitude about protecting customers
data.
The company takes security measures seriously.

The company is the victim of the data breach.
The company had a relaxed attitude about protecting customers
The company takes security measures seriously.

The company is the victim of the data breach.
The company had a relaxed attitude about protecting customers
data.
The company takes security measures seriously. Strongly
disagree Disagree Neutral Agree $\begin{gathered}\text { Strongly } \\ \text { agree }\end{gathered}$

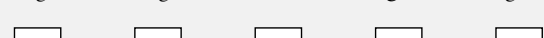

Attackers wanted to harm the company.

Attackers wanted to harm the customers.

The company is helping customers recover from the breach.

The company put customers at risk by neglecting data protection.

The company is accountable for problems resulting from the data breach.

The customers are accountable for problems resulting from the data breach.
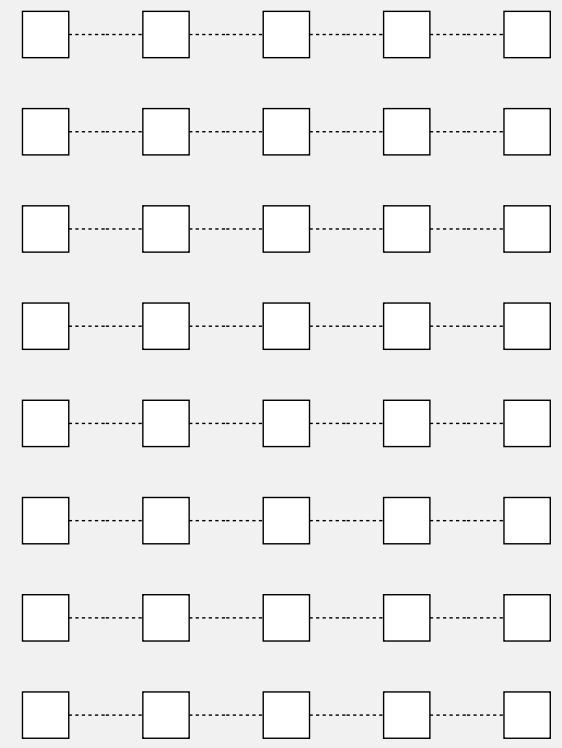
F2. Read the following extract carefully and indicate the extent to which you agree or disagree with the following statements. Company B Data Breach Statement:

"The company's business - its only business - is to collect, securely store and aggregate information on more than 800 million individual consumers and nearly $90 \mathrm{~m}$ businesses worldwide... In the three years before the data breach, the company spent only about $3 \%$ of its operating revenue on cybersecurity-less than the company spent on stock dividends. This company and other big agencies keep profiting off a business model that rewards their failure to protect personal information. The company was breached in "mid-May" 2017, realized it in July and got around to telling the world in early September. As the company continues to issue incomplete, conf using and contradictory statements and hide information from Congress and the public, it is clear that five months after the breach was publicly announced, The company has yet to answer this simple question in full: what was the precise extent of the breach? The consumer complaints about improper use of credit reports, incorrect information on credit reports, inadequate assistance in resolving problems, and problems with the company credit monitoring, fraud alerts, and security freezes in the wake of the breach."

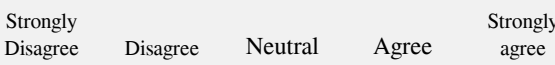

The customers of the company are victims of the data breach.

The company is the victim of the data breach.

The company had a relaxed attitude about protecting customers data.

The company takes security measures seriously. Attackers wanted to harm the company.

Attackers wanted to harm the customers.

The company is helping customers recover from the breach.

The company put customers at risk by neglecting data protection.

The company is accountable for problems resulting from the data breach.
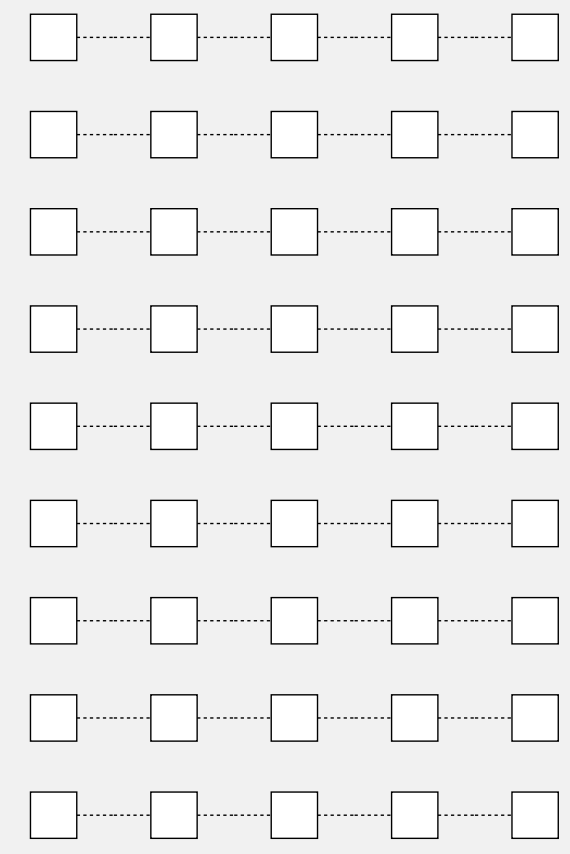

The customers are accountable for problems resulting from the data breach.

F3. Q 


\section{Section G: Data Breach Description}

G1. Read the following extract carefully and indicate the extent to which you agree or disagree with the following statements. Company A Data Breach Statement:

“Company A today announced a cybersecurity incident potentially impacting approximately 143 million consumers. Criminals exploited a website application vulnerability to gain access to certain files. The company discovered the unauthorized access on July 29 of this year and acted immediately to stop the intrusion. The company promptly engaged a leading, independent cybersecurity firm that has been conducting a comprehensive forensic review to determine the scope of the intrusion, including the specific data impacted. "We pride ourselves on being a leader in managing and protecting data, and we are conducting a thorough review of our overall security operations. We also are focused on consumer protection and have developed a comprehensive portfolio of services to support all U.S. consumers, regardless of whether they were impacted by this incident." said the Chairman and Chief Executive Officer of the company. The company has established a dedicated website to help consumers determine if their information has been potentially impacted and to sign up for credit file monitoring and identity theft protection."

The customers of the company are victims of the data breach.

The company is the victim of the data breach.

The company had a relaxed attitude about protecting customers
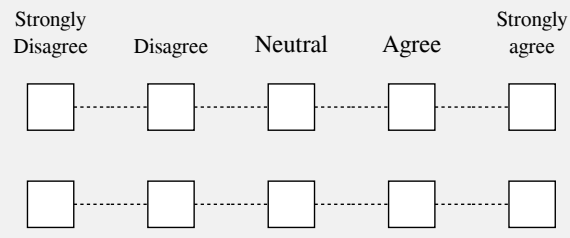

\section{data. \\ The company takes security measures seriously. \\ Attackers wanted to harm the company. \\ Attackers wanted to harm the customers.}
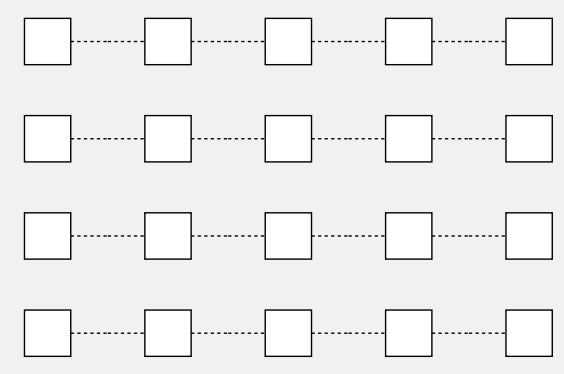

The company is helping customers recover from the breach.

The company put customers at risk by neglecting data protection.

The company is accountable for problems resulting from the data breach.

The customers are accountable for problems resulting from the data breach.
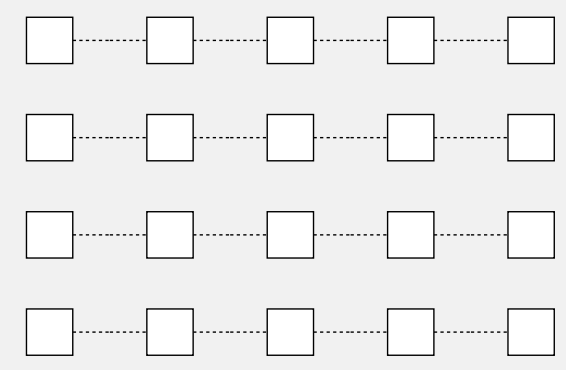
G2. Read the following extract carefully and indicate the extent to which you agree or disagree with the following statements. Company B Data Breach Statement:

'It's been almost two weeks since the company first admitted it had been hacked in a massive breach affecting as many as 143 million consumers. Ever since, people have been begging the company to answer a simple question: "Am I on the list of victims?" The company doesn't face the constant monitoring and auditing that help strengthen banks' systems and data protections. Despite the wealth of sensitive information in its databases, the company, in essence, falls through the regulatory cracks. When the company told the world on Sept. 7 that thieves had helped themselves to as many as 143 million Social Security numbers, it sent people to a website for more information. The website barely functioned. Those who turned to the phones overwhelmed call centers, and people who did get through found that most representatives were not helpful. To make matters worse, some of those centers were right in the path of Hurricane Irma."

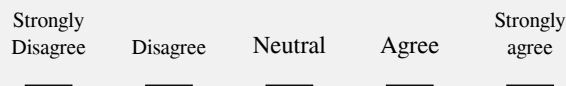

The customers of the company are victims of the data breach.

The company is the victim of the data breach.

The company had a relaxed attitude about protecting customers data.

The company takes security measures seriously.

Attackers wanted to harm the company.

Attackers wanted to harm the customers.

The company is helping customers recover from the breach.

The company put customers at risk by neglecting data protection.

The company is accountable for problems resulting from the data breach.
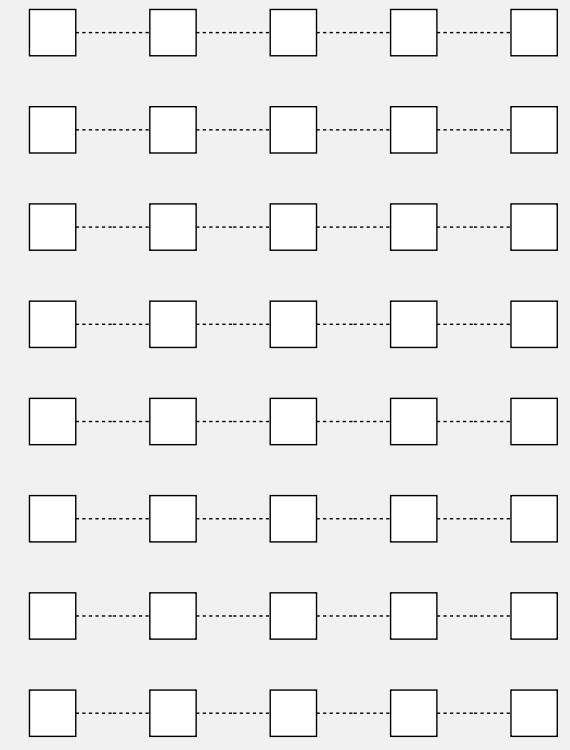

The customers are accountable for problems resulting from the data breach.

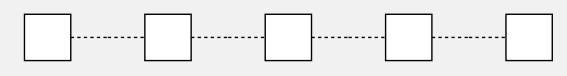

G3. Q 


\section{Section H: Data Breach Description}

H1. Read the following extract carefully and indicate the extent to which you agree or disagree with the following statements. Company A Data Breach Statement:

"Company A values our guests and understands the importance of protecting personal information. We have taken measures to investigate and address a data security incident involving the database. The investigation has determined that there was unauthorized access to the database, which contained guest information relating to reservations at the properties on or before September 10, 2018. The company deeply regrets this incident happened. From the start, we moved quickly to contain the incident and conduct a thorough investigation with the assistance of leading security experts. The company is working hard to ensure our guests have answers to questions about their personal information with a dedicated website and call center. We are supporting the efforts of law enforcement and working with leading security experts to improve. The company is also devoting the resources necessary to phase out the systems and accelerate the ongoing security enhancements to our network. The company is providing guests the opportunity to enroll in WebWatcher free of charge for one year. WebWatcher monitors internet sites where personal information is shared and generates an alert to the consumer if evidence of the consumer's personal information is found."

The customers of the company are victims of the data breach.

The company is the victim of the data breach.

The company had a relaxed attitude about protecting customers data.

The company takes security measures seriously.

Attackers wanted to harm the company.

Attackers wanted to harm the customers.

The company is helping customers recover from the breach.

The company put customers at risk by neglecting data protection.

The company is accountable for problems resulting from the data breach.

The customers are accountable for problems resulting from the data breach.
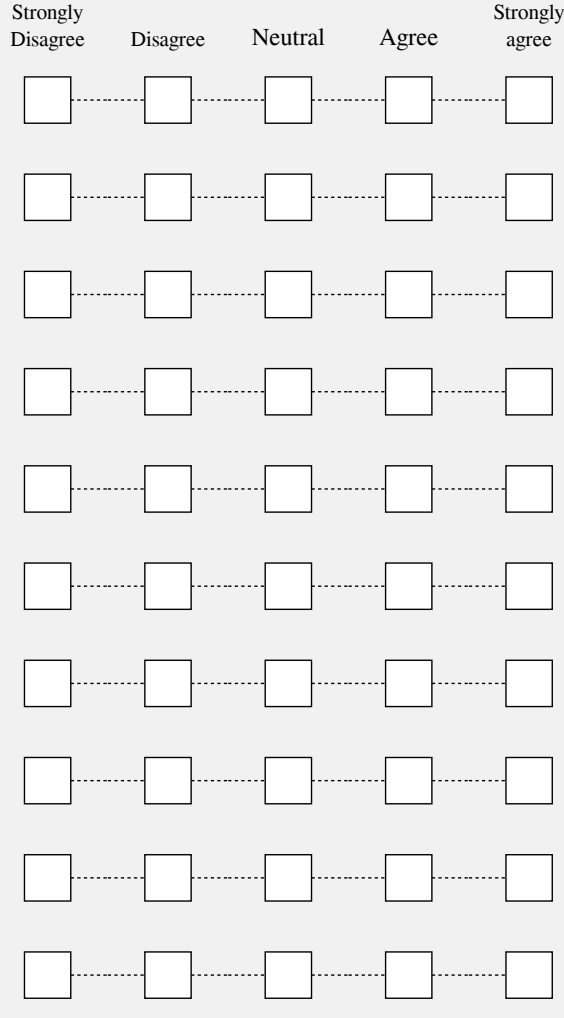
H2. Read the following extract carefully and indicate the extent to which you agree or disagree with the following statements. Company B Data Breach Statement:

"The cybercriminals gained access to, copied and encrypted a wide variety of data from guests using its reservation system, the company said. The company IT team only discovered the breach on September 8, 2018, when the cybercriminals attempted to remove data from the system. The company said the miscreants who broke into its database made off with a total of $\mathbf{5 . 2 5}$ million unencrypted passport numbers and 20.3 million encrypted numbers. A key question we need to ask is why do hotels need to store passport numbers? One of the biggest impacts of new regulation on data protection was that it forced companies to consider whether the personal data they hold and ask customers for... was really needed and if so how to properly protect it. This event led to a further investigation which uncovered that the long-running operation had been in place since 2014. Since their presence was in place two years before the company's purchase of hotels there was an obvious omission by the company during its vetting process of the system and its computer network. Moreover, the company was no stranger to data breaches, having been hit in 2015 with POS malware affecting 1,275 properties in the U.S. and Canada."

Strongly
Disagree $\quad$ Disagree Neutral Agree $\begin{gathered}\text { Strongly } \\ \text { agree }\end{gathered}$

The customers of the company are victims of the data breach.

The company is the victim of the data breach.

The company had a relaxed attitude about protecting customers data.

The company takes security measures seriously.

Attackers wanted to harm the company.

Attackers wanted to harm the customers.

The company is helping customers recover from the breach.

The company put customers at risk by neglecting data protection.

The company is accountable for problems resulting from the data breach.

The customers are accountable for problems resulting from the

data breach.
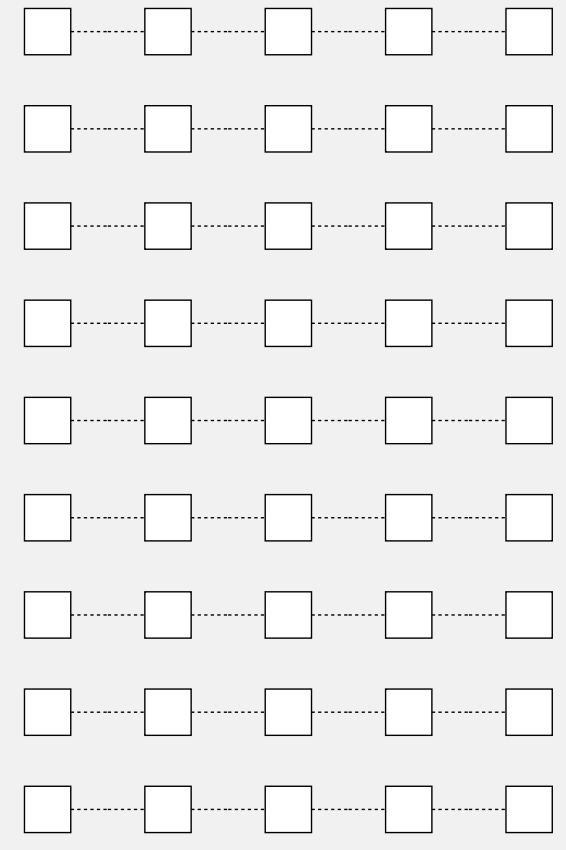


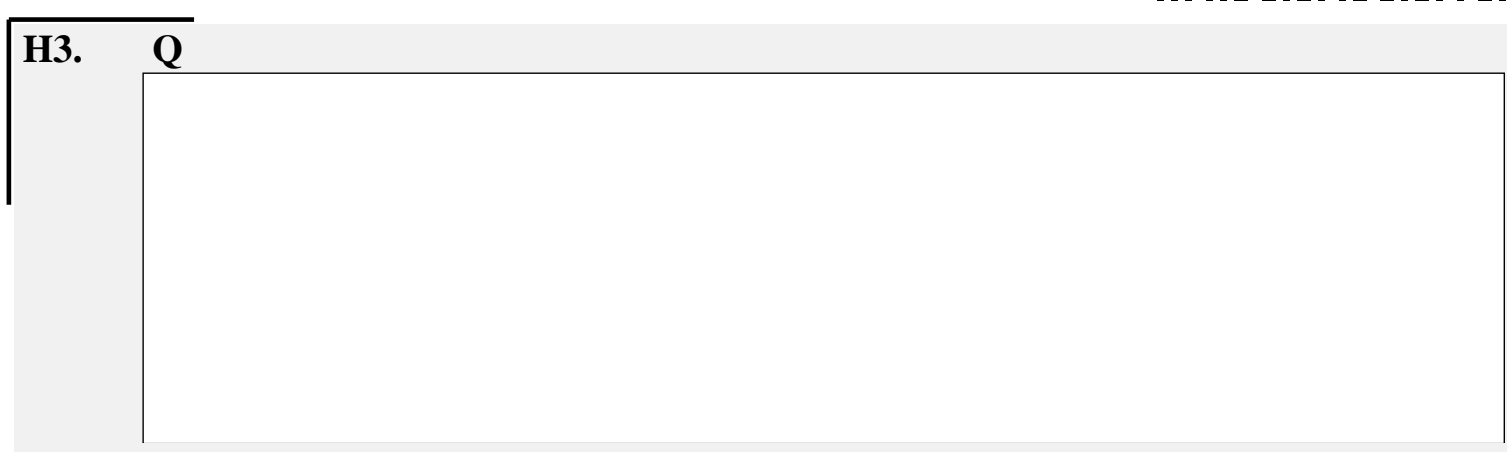

Secret Completion Code: HC99

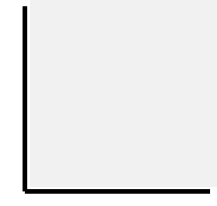

